\title{
Topological Logics with Connectedness over Euclidean Spaces
}

\author{
Roman Kontchakov Yavor Nenov Ian Pratt-Hartmann \\ Michael Zakharyaschev
}

\begin{abstract}
We consider the quantifier-free languages, $\mathcal{B} c$ and $\mathcal{B} c^{\circ}$, obtained by augmenting the signature of Boolean algebras with a unary predicate representing, respectively, the property of being connected, and the property of having a connected interior. These languages are interpreted over the regular closed sets of $\mathbb{R}^{n}(n \geq 2)$ and, additionally, over the regular closed polyhedral sets of $\mathbb{R}^{n}$. The resulting logics are examples of formalisms that have recently been proposed in the Artificial Intelligence literature under the rubric Qualitative Spatial Reasoning. We prove that the satisfiability problem for $\mathcal{B} c$ is undecidable over the regular closed polyhedra in all dimensions greater than 1 , and that the satisfiability problem for both languages is undecidable over both the regular closed sets and the regular closed polyhedra in the Euclidean plane. However, we also prove that the satisfiability problem for $\mathcal{B} c^{\circ}$ is $\mathrm{NP}$-complete over the regular closed sets in all dimensions greater than 2 , while the corresponding problem for the regular closed polyhedra is ExPTIME-complete. Our results show, in particular, that spatial reasoning over Euclidean spaces is much harder than reasoning over arbitrary topological spaces.
\end{abstract}

\section{Introduction}

Let $\mathcal{B} c$ be the quantifier-free fragment of first-order logic in the signature $(+, \cdot,-, 0,1, c)$, where $c$ is a unary predicate; and let $\operatorname{RCP}\left(\mathbb{R}^{n}\right)$ be the collection of regular closed, polyhedral sets in $n$-dimensional Euclidean space. (A set is regular closed if it is the closure of an open set, and polyhedral if it is a finite union of finite intersections of closed half-spaces.) The collection $\operatorname{RCP}\left(\mathbb{R}^{n}\right)$ forms a Boolean algebra under the subset ordering; and we interpret $\mathcal{B} c$ over $\operatorname{RCP}\left(\mathbb{R}^{n}\right)$ by taking the symbols $+, \cdot,-, 0,1$ to have their natural meanings in this Boolean algebra, and by taking $c$ to denote the property of being connected. Intuitively, we think of elements of $\operatorname{RCP}\left(\mathbb{R}^{n}\right)$ as regions of space, and of formulas of $\mathcal{B} c$ as descriptions of arrangements of these regions. Our primary concern is the satisfiability problem: given a $\mathcal{B} c$-formula, is there an assignment of elements of $\operatorname{RCP}\left(\mathbb{R}^{n}\right)$ to its variables making it true?

The motivation for studying this problem comes from the field of Qualitative 
Spatial Reasoning in Artificial Intelligence, the aim of which is to develop formal languages for representing and processing qualitative spatial information. In this context, $\mathcal{B} c$ constitutes a parsimonious language: it has no quantifiers, and its non-logical primitives express only the basic region-combining operations and the property of connectedness. At the same time, the structures $\operatorname{RCP}\left(\mathbb{R}^{n}\right)$ - particularly in the cases $n=2$ and $n=3$ - constitute its most natural domains of interpretation, given current practice in the fields of Qualitative Spatial Reasoning, Geographic Information Systems and Spatial Databases.

For reasons discussed below, we broaden the subject of enquiry slightly. Let $\mathcal{B} c^{\circ}$ denote the quantifier-free fragment of first-order logic in the signature $\left(+, \cdot,-, 0,1, c^{\circ}\right)$, where $+, \cdot,-, 0,1$ are as before, and $c^{\circ}$ is a unary predicate interpreted as the property of having a connected interior. Further, let $\mathrm{RC}\left(\mathbb{R}^{n}\right)$ denote the collection of regular closed sets in $n$-dimensional Euclidean space. Again, $\mathrm{RC}\left(\mathbb{R}^{n}\right)$ forms a Boolean algebra under the subset ordering, having $\operatorname{RCP}\left(\mathbb{R}^{n}\right)$ as a sub-algebra. Intuitively, we think of $\mathrm{RC}\left(\mathbb{R}^{n}\right)$ as a more liberal model of spatial regions than $\operatorname{RCP}\left(\mathbb{R}^{n}\right)$. In the sequel, we consider the satisfiability problem for $\mathcal{B} c$ and $\mathcal{B} c^{\circ}$ over the structures $\operatorname{RC}\left(\mathbb{R}^{n}\right)$ and $\operatorname{RCP}\left(\mathbb{R}^{n}\right)$. The results of this paper are as follows: $(i)$ the satisfiability problem for $\mathcal{B} c$ over $\operatorname{RCP}\left(\mathbb{R}^{n}\right)$ is undecidable for all $n \geq 2$; (ii) the satisfiability problem for $\mathcal{B} c$ over $\mathrm{RC}\left(\mathbb{R}^{2}\right)$ is undecidable, as are the satisfiability problems for $\mathcal{B} c^{\circ}$ over both $\mathrm{RC}\left(\mathbb{R}^{2}\right)$ and $\mathrm{RCP}\left(\mathbb{R}^{2}\right)$; (iii) the satisfiability problem for $\mathcal{B} c^{\circ}$ over $\operatorname{RC}\left(\mathbb{R}^{n}\right)$ is NP-complete for all $n \geq 3$, while over $\operatorname{RCP}\left(\mathbb{R}^{n}\right)$ the corresponding problem is ExPTIME-complete. (It may be of interest to note that, over $\mathrm{RC}(\mathbb{R})$ and $\operatorname{RCP}(\mathbb{R})$, the satisfiability problem for $\mathcal{B} c$ and $\mathcal{B} c^{\circ}$ is NP-complete.) The decidability of the satisfiability problems for $\mathcal{B} c$ over $\operatorname{RC}\left(\mathbb{R}^{n}\right)$, for $n \geq 3$, is left open. Results (ii) and (iii) were announced, without proofs, in [28, 24].

Mathematically, it is also meaningful to consider the satisfiability of $\mathcal{B c}$ - and $\mathcal{B} c^{\circ}$-formulas over the regular closed subsets of any topological space. If $T$ is a topological space, we denote the collection of regular closed subsets of $T$ by $\mathrm{RC}(T)$; again, this collection always forms a Boolean algebra under the subset ordering. The satisfiability problem for $\mathcal{B} c$ over the class of structures of the form $\operatorname{RC}(T)$ is known to be ExPTime-complete, while for $\mathcal{B} c^{\circ}$, the corresponding problem is NP-complete [27, 28. However, satisfiability over arbitrary topological spaces is of at most marginal relevance to Qualitative Spatial Reasoning. Indeed, the results reported here show that, for languages able to express the property of connectedness, reasoning over Euclidean spaces is a different kettle of fish altogether. In the remainder of this section, we discuss the significance of these results in the context of recent developments in spatial, algebraic and modal logics.

\subsection{Spatial logic}

A spatial logic is a formal language interpreted over some class of geometrical structures. Spatial logics, thus understood, have a long history, tracing their origins back both to the axiomatic tradition in geometry [23, 48, and also the region-based theory of space [52, 12, subsequently developed in [7, 8, 4]. Such 
logics were proposed as a formalism for Qualitative Spatial Reasoning in the seminal paper [40. The basic idea is as follows: numerical coordinate-based descriptions of the objects that surround us are hard to acquire, inherently error-prone, and probably unnecessary for everyday spatial reasoning tasks; therefore - so goes the argument - we should employ a representation language whose variables range over spatial regions (rather than points), and whose nonlogical primitives are interpreted as qualitative (rather than quantitative) relations and operations. On this view, formulas are to be understood as expressing descriptions of (putative) configurations of objects in space, with the satisfiability of a formula over the space in question equating to the geometrical realizability of the described arrangement. If we imagine an intelligent agent employing such a language to represent spatial arrangements of objects, then the problem of recognizing satisfiable formulas amounts to that of eliciting the geometrical knowledge latent in that agent's operating environment and cognitive design.

The best-known, and most intensively studied, qualitative spatial representation language is $\mathcal{R C C} 8$ [17, 40, 47. This language features predicates for the six topological relations DC (disconnection), EC (external connection), PO (partial overlap), EQ (equality), TPP (tangential proper part) and NTPP (nontangential proper part) illustrated, for the case of closed disc-homeomorphs, in Fig. 1. (The name $\mathcal{R C C} 8$ becomes less puzzling when we observe that the relations TPP and NTPP are asymmetric.) Note that $\mathcal{R C C} 8$ has no individual constants or function symbols, and no quantifiers. Traditionally, $\mathcal{R C C} 8$ is in-

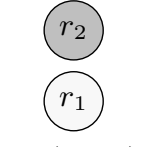

$\mathrm{DC}\left(r_{1}, r_{2}\right)$

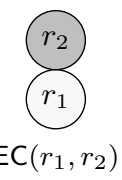

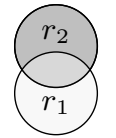

$\mathrm{PO}\left(r_{1}, r_{2}\right)$

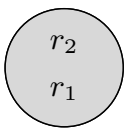

$\mathrm{EQ}\left(r_{1}, r_{2}\right)$

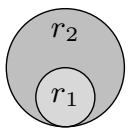

$\operatorname{TPP}\left(r_{1}, r_{2}\right) \operatorname{NTPP}\left(r_{1}, r_{2}\right)$

Figure 1: $\mathcal{R C C} 8$-relations over discs in $\mathbb{R}^{2}$.

terpreted over the regular closed sets in some topological space, so as to finesse the awkward issue of whether regions should be taken to include their boundary points. The satisfiability problems for $\mathcal{R C C} 8$ over the class of structures of the form $\mathrm{RC}(T)$ (for $T$ a topological space) is easily seen to be NP-complete, though tractable fragments have been explored 44, 21. Further, satisfiability of an $\mathcal{R C C} 8$ formula over any structure in this class implies satisfiability over $\operatorname{RCP}\left(\mathbb{R}^{n}\right)$, for all $n \geq 1$ [4]. Thus, the satisfiability problems for $\operatorname{RC}\left(\mathbb{R}^{n}\right)$ and $\operatorname{RCP}\left(\mathbb{R}^{n}\right)(n \geq 1)$ coincide and are NP-complete - a fact which testifies to the restricted expressive power of $\mathcal{R C C} 8$.

A word of caution is in order at this point. Satisfiability of an $\mathcal{R C C} 8$ formula over $\mathrm{RC}\left(\mathbb{R}^{2}\right)$ does not necessarily imply satisfiability by natural or $f a$ miliar regions - for example, closed disc-homeomorphs. The $\mathcal{R C C} 8$-satisfiability problem for such interpretations requires specialized, and highly non-trivial, techniques. A landmark result [45] in the area shows, however, that the satisfiability problem for $\mathcal{R C C} 8$ interpreted over the closed disc-homeomorphs in $\mathbb{R}^{2}$ is 
still in NP. The contribution of present paper, with its emphasis on Euclidean spaces and the property of connectedness, imposes severe limits on what further results of this kind we can hope for.

We mentioned above that, if $T$ is a topological space, the collection $\operatorname{RC}(T)$ always forms a Boolean algebra under the subset ordering. This enables us to extend $\mathcal{R C C} 8$ with the function symbols $+, \cdot,-$ and constants 0,1 , interpreting these in the natural way over any structure $\mathrm{RC}(T)$. Such an extended language was originally introduced in $[53$ under the name $\mathcal{B R C C} 8$ (Boolean $\mathcal{R C C} 8$ ). Intuitively, if $a_{1}$ and $a_{2}$ are regular closed sets, we may think of $a_{1}+a_{2}$ as the agglomeration of $a_{1}$ and $a_{2}, a_{1} \cdot a_{2}$ as the common part of $a_{1}$ and $a_{2},-a_{1}$ as the complement of $a_{1}, 0$ as the empty region and 1 as the whole space. The satisfiability problem for $\mathcal{B R C C} 8$ over the class of structures of the form $\operatorname{RC}(T)$ is still NP-complete; however, restricting attention to connected spaces $T$ yields a PSPACE-complete satisfiability problem. Thus, $\mathcal{B R C C} 8$, unlike $\mathcal{R C C} 8$, has sufficient expressive power to distinguish between satisfiability over arbitrary spaces and satisfiability over connected spaces. But that is about as far as this extra expressive power takes us: satisfiability of a $\mathcal{B R C C} 8$-formula over any structure $\operatorname{RC}(T)$, for $T$ connected, implies satisfiability over $\operatorname{RCP}\left(\mathbb{R}^{n}\right)$ for all $n \geq 1$. Hence, the satisfiability problems for $\mathrm{RC}\left(\mathbb{R}^{n}\right)$ and $\operatorname{RCP}\left(\mathbb{R}^{n}\right)(n \geq 1)$ coincide, and are PSPACE-complete. Note in particular that $\mathcal{B R C C} 8$ does not enable us to say that a given region of space is connected.

We end this discussion of $\mathcal{R C C} 8$ and $\mathcal{B R C C} 8$ with a remark on the absence of quantification from these languages. This restriction is motivated by computability considerations: essentially all region-based spatial logics with full first-order syntax have undecidable satisfiability problems, and so are considered unsuitable for Qualitative Spatial Reasoning [22, 15, 11, 32. To be sure, first-order spatial logics are nevertheless of considerable model-theoretic interest; see [39] for a survey. We note in particular that, if we can quantify over regions, then the $\mathcal{R C C} 8$-primitives easily enable us to define, over most interesting classes of interpretations, all of the primitives $+, \cdot,-, 0,1, c$ and $c^{\circ}$. However, as computability considerations are to the fore in this paper, we too confine ourselves to quantifier-free formalisms in the sequel.

\section{$1.2 \quad$ Algebraic and modal logic}

The standard view of topology takes a topological space to consist of a set of points on which a collection of open subsets is defined. However, a dual view is possible, in which one begins with a Boolean algebra, and then adds algebraic structure defining distinctively topological relations between its objects. There are two main approaches to developing this second view. On the first, we think of the underlying Boolean algebra as a field of sets, and we augment this Boolean algebra with a pair of unary operators, conceived of as representing the operations of closure and interior, and assumed to obey the standard Kuratowski axioms 33. The striking similarity between these axioms and the axioms for the propositional modal logic $\mathcal{S} 4$ [36, 19] led to the development of topological semantics for modal logics. Under this semantics, the (proposi- 
tional) variables are taken to range over any collection of subsets of a topological space (not just regular closed sets), and the logical connectives are interpreted by the operations of union, intersection, complement and topological interior (for necessity) and closure (for possibility). The extension of this language with the universal modality, denoted $\mathcal{S} 4_{u}$ [20], is known to be a super-logic for $\mathcal{R C C} 8$ and $\mathcal{B R C C} 8$ [3, 42, 9, 35, 53. The satisfiability problem for $\mathcal{S} 4_{u}$ is the same over every connected, separable, dense-in-itself metric space, and this problem is PSPACE-complete [33, 46, 1. We remark that, as for $\mathcal{R C C} 8$ and $\mathcal{B R C C} 8, \mathcal{S} 4_{u}$ is unable to express the condition that a region is connected. For a survey of the relationship between spatial and modal logics see [51, 18, 26] and references therein.

On the second approach, we instead think of the underlying Boolean algebra as an algebra of regular closed sets, and we augment this Boolean algebra with a binary predicate $C$, conceived of as representing the relation of contact. (Two sets are said to be in contact if they have a non-empty intersection). This binary predicate is assumed to satisfy the axioms of contact algebras, a category which is known to be dual to the category of dense sub-algebras of regular closed algebras of topological spaces [16, 13, 14, 2, 49, 50. The contact relation as a basis for topology actually has a venerable career, having originally been introduced in [52] under the name 'extensive connection'. More relevantly for the present paper, it is straightforward to show that all the $\mathcal{R C C} 8$ relations can be expressed, in purely propositional terms, using this signature [2, 26]. (Thus, for example, $\operatorname{EC}\left(\tau_{1}, \tau_{2}\right)$ is equivalent to $C\left(\tau_{1}, \tau_{2}\right) \wedge\left(\tau_{1} \cdot \tau_{2}=0\right)$.) For this reason, we regard the propositional language over the signature $(+, \cdot,-, 0,1, C)$, here denoted $\mathcal{C}$, as equivalent to the language $\mathcal{B R C C} 8$ mentioned above. The purely Boolean fragment of $\mathcal{C}$ (without the contact predicate $C$ ) is denoted by $\mathcal{B}$. This language is in fact equivalent to the extension of the spatial logic $\mathcal{R C C} 5[3]$ with the function symbols,+ and - .

\subsection{Spatial logics with connectedness}

Most spatial regions of interest - plots of land in a cadastre, the space occupied by physical objects, paths swept out by moving objects - are either connected or at least contain few connected components [10. It seems, therefore, that to be genuinely useful, logics for Qualitative Spatial Reasoning should possess some means of expressing this notion. The simplest way of proceeding is to consider languages featuring a unary predicate denoting this property. Various such languages have been investigated before [38, 25, 27, 50, 49]; the language $\mathcal{B} c$ is chosen for study here because it is so parsimonious.

It is worth bearing in mind, however, that 'connectedness,' in the topologists' sense may not be exactly what we want. For example, a region consisting of two closed discs externally touching is, in this sense, connected, yet, in certain contexts, may be functionally equivalent to a disconnected region. (Imagine having a garden that shape.) In such contexts, it may be more useful to employ the notion of a region's having a connected interior, a property we refer to as interior-connectedness. Note that every regular closed, interior-connected set 
is connected; also, in the space $\mathbb{R}$, the notions of connectedness and interiorconnectedness coincide. So as not to prejudge the issue here, we employ predicates for both notions: $c$ to denote the standard property of connectedness, $c^{\circ}$ to denote the property of interior-connectedness. Hence, in addition to the 'minimal' language $\mathcal{B} c$, we have its counterpart $\mathcal{B} c^{\circ}$.

Strikingly, the languages $\mathcal{R C C} 8$ and $\mathcal{B R C C} 8$, which cannot represent connectedness (or, for that mater, interior-connectedness), are far less sensitive to the underlying geometrical interpretation than the languages $\mathcal{B} c$ and $\mathcal{B} c^{\circ}$, which can. For example, an $\mathcal{R C C} 8$-formula that is satisfiable over the regular closed algebra of any topological space is satisfiable over $\operatorname{RCP}\left(\mathbb{R}^{n}\right)$, for all $n \geq 1$ [41]. Or again, a $\mathcal{B R C C} 8$-formula that is satisfiable over the regular closed algebra of any connected topological space is satisfiable over $\operatorname{RCP}\left(\mathbb{R}^{n}\right)$, for all $n \geq 1[53$. Thus, $\mathcal{R C C} 8$ and $\mathcal{B R C C} 8$ care neither about the dimension of the (Euclidean) space we are reasoning about, nor about the distinction between regular closed polyhedra and arbitrary regular closed sets. Not so with the languages $\mathcal{B} c$ or $\mathcal{B} c^{\circ}$, which are sensitive both to the dimension of space and to the restriction to polyhedral regular closed sets. This sensitivity is easy to demonstrate for $\mathcal{B} c^{\circ}$, and we briefly do so here, by way of illustration.

Consider first sensitivity to dimension. The $\mathcal{B} c^{\circ}$-formula

$$
\bigwedge_{1 \leq i \leq 3}\left(c^{\circ}\left(r_{i}\right) \wedge\left(r_{i} \neq 0\right)\right) \wedge \bigwedge_{1 \leq i<j \leq 3}\left(c^{\circ}\left(r_{i}+r_{j}\right) \wedge\left(r_{i} \cdot r_{j}=0\right)\right)
$$

'says' that $r_{1}, r_{2}$ and $r_{3}$ are non-empty regions with connected interiors, such that each forms an interior-connected sum with the other two, without overlapping them. It is obvious that this formula is not satisfiable over $\mathrm{RC}(\mathbb{R})$. For the non-empty, (interior-) connected regular closed sets on the real line are precisely the non-punctual, closed intervals, and it is impossible for three such intervals to touch each other without overlapping. On the other hand, (1) is easily seen to be satisfiable over $\mathrm{RC}\left(\mathbb{R}^{n}\right)$ for all $n \geq 2$. Likewise, the $\mathcal{B} c^{\circ}$-formula

$$
\bigwedge_{1 \leq i \leq 5}\left(c^{\circ}\left(r_{i}\right) \wedge\left(r_{i} \neq 0\right)\right) \wedge \bigwedge_{1 \leq i<j \leq 5}\left(c^{\circ}\left(r_{i}+r_{j}\right) \wedge\left(r_{i} \cdot r_{j}=0\right)\right)
$$

which makes the analogous claim for regions $r_{1}, \ldots, r_{5}$, is not satisfiable over $\mathrm{RC}\left(\mathbb{R}^{2}\right)$, since any satisfying assignment would permit a plane drawing of the graph $K_{5}$. On the other hand, (2) is easily seen to be satisfiable over $\operatorname{RC}\left(\mathbb{R}^{n}\right)$ for all $n \geq 3$. Thus, the satisfiability problems for $\mathcal{B} c^{\circ}$ over $\mathrm{RC}(\mathbb{R}), \operatorname{RC}\left(\mathbb{R}^{2}\right)$ and $\mathrm{RC}\left(\mathbb{R}^{3}\right)$ are all different. (We shall see in Sec. 6, however, that the satisfiability problem for $\mathcal{B} c^{\circ}$ over $\mathrm{RC}\left(\mathbb{R}^{n}\right)$ is the same for all $n \geq 3$.)

Consider next sensitivity to restriction to (regular closed) polyhedral sets. The $\mathcal{B} c^{\circ}$-formula

$$
\bigwedge_{1 \leq i \leq 3} c^{\circ}\left(r_{i}\right) \wedge c^{\circ}\left(r_{1}+r_{2}+r_{3}\right) \wedge \bigwedge_{2 \leq i \leq 3} \neg c^{\circ}\left(r_{1}+r_{i}\right)
$$

is satisfiable over $\mathrm{RC}\left(\mathbb{R}^{2}\right)$, as we see from the regular closed sets in Fig. 2 , where $r_{2}$ and $r_{3}$ lie, respectively, above and below the graph of the function $\sin \frac{1}{x}$ on 
the interval $(0,1]$. By contrast, formula (3) is unsatisfiable over $\operatorname{RCP}\left(\mathbb{R}^{n}\right)$ for all $n \geq 1$ [27. Actually, the result can be sharpened: (3) is unsatisfiable over

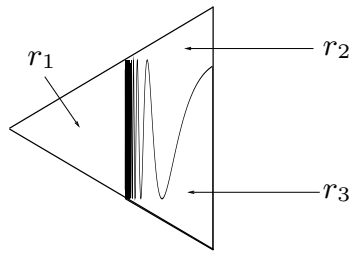

Figure 2: Three regions in $\mathrm{RC}\left(\mathbb{R}^{2}\right)$ satisfying (3).

any Boolean sub-algebra of $\mathrm{RC}\left(\mathbb{R}^{n}\right)$ whose regions all satisfy a form of the curve selection lemma from real algebraic geometry (see, e.g. [5]). As we might say, in dimensions 2 and above, $\mathcal{B} c^{\circ}$ is sensitive to the presence of 'non-tame' regions. And since - at least conceivably - non-tame regions may be thought implausible models of the space occupied by any physical objects - it is natural to consider satisfiability of $\mathcal{B} c^{\circ}$-formulas over $\operatorname{RCP}\left(\mathbb{R}^{n}\right)$ rather than over $\operatorname{RC}\left(\mathbb{R}^{n}\right)$.

The language $\mathcal{B} c$ is similarly sensitive to the dimension of the Euclidean space over which it is interpreted, and also to the restriction to polyhedral regions. For dimensionality, this sensitivity can be demonstrated by examples similar to (1) and (2); see 26. For the restriction to polyhedral regions, this result follows from Sec. 3. where we show that there exists a $\mathcal{B} c$-formula satisfiable in $\mathrm{RC}\left(\mathbb{R}^{n}\right)$ for all $n \geq 2$, but only by tuples of regions having infinitely many connected components!

\subsection{Plan of the paper and summary of results}

The remainder of this paper is organized as follows. Sec. 2 defines the syntax and semantics of $\mathcal{B} c$ and $\mathcal{B} c^{\circ}$. To simplify proofs, we also employ the more expressive languages $\mathcal{C} c$ and $\mathcal{C} c^{\circ}$, obtained by adding the predicates $c$ and $c^{\circ}$, respectively, to $\mathcal{C}(=\mathcal{B R C C} 8)$. In $\mathrm{Sec}$. 3 we prove that there exist $\mathcal{C} c_{-}, \mathcal{C}^{\circ}{ }^{\circ}$ and $\mathcal{B} c$-formulas satisfiable over $\mathrm{RC}\left(\mathbb{R}^{n}\right)$, for all $n \geq 1$, but only by tuples of regions some of which have infinitely many connected components, and hence which cannot belong to $\operatorname{RCP}\left(\mathbb{R}^{n}\right)$. By further developing the ideas encountered in this proof, we show in Sec. 4 that $\mathcal{C} c, \mathcal{C} c^{\circ}$ and $\mathcal{B} c$ (but not $\mathcal{B} c^{\circ}$ ) are r.e.hard over $\operatorname{RCP}\left(\mathbb{R}^{n}\right)$, for all $n \geq 2$. Using a different approach, we show in Sec. 5 that all four of our $\operatorname{logics}-\mathcal{B} c, \mathcal{B} c^{\circ}, \mathcal{C} c$ and $\mathcal{C} c^{\circ}$ - are r.e.-hard over both $\operatorname{RCP}\left(\mathbb{R}^{2}\right)$ and $\mathrm{RC}\left(\mathbb{R}^{2}\right)$. Finally, we show in Sec. 6 that $\mathcal{B} c^{\circ}$ is $\mathrm{NP}$-complete over $\operatorname{RC}\left(\mathbb{R}^{n}\right)$, and ExPTIME-complete over $\operatorname{RCP}\left(\mathbb{R}^{n}\right)$, for all $n \geq 3$. The decidability of satisfiability for $\mathcal{C} c, \mathcal{C} c^{\circ}$ and $\mathcal{B} c$ over $\operatorname{RC}\left(\mathbb{R}^{n}\right)$, for all $n \geq 3$, is left open. 


\section{Preliminaries}

We begin by formally defining the syntax and semantics of the topological logics considered in this paper. This section also contains the basic technical definitions and results we need in what follows.

\subsection{Basic topological notions}

A topological space is a pair $(T, \mathcal{O})$, where $T$ is a set and $\mathcal{O}$ a collection of subsets of $T$ containing $\emptyset$ and $T$, and closed under arbitrary unions and finite intersections. The elements of $\mathcal{O}$ are referred to as open sets; their complements are closed sets. If $\mathcal{O}$ is clear from context, we refer to the topological space $(T, \mathcal{O})$ simply as $T$. If $X \subseteq T$, the closure of $X$, denoted $X^{-}$, is the smallest closed set including $X$, and the interior of $X$, denoted $X^{\circ}$, is the largest open set included in $X$. These sets always exist. The boundary of $X$, denoted $\delta X$, is the set $X^{-} \backslash X^{\circ}$. The Euclidean space $\mathbb{R}^{n}$ is assumed always to have the usual metric topology. We may treat any subset $X \subseteq T$ as a topological space in its own right by defining the subspace topology on $X$ to be the collection of sets $\mathcal{O}_{X}=\{O \cap X \mid O \in \mathcal{O}\}$.

We call $X$ regular closed if it is the closure of an open set-equivalently, if $X=\left(X^{\circ}\right)^{-}$. We denote by $\mathrm{RC}(T)$ the set of regular closed subsets of $T$. It is a standard result that $\mathrm{RC}(T)$ forms a complete Boolean algebra, with operations $\sum A=(\bigcup A)^{-}, \prod A=(\bigcap A)^{\circ-}$ and $-X=(T \backslash X)^{-}$(see, e.g. 29]). The partial order induced by this Boolean algebra is simply $(T, \subseteq)$; we often write $X \leq Y$ in preference to $X \subseteq Y$ where $X$ and $Y$ are regular closed. Note that, if $A=\left\{X_{1}, X_{2}\right\}$, then $\sum A=X_{1}+X_{2}=X_{1} \cup X_{2}$.

A topological space $T$ is said to be connected if it cannot be decomposed into two disjoint, non-empty closed sets; likewise, $X$ is connected if it is a connected space under the subspace topology. We call $X$ interior-connected if $X^{\circ}$ is connected. A maximal connected subset of $X$ will be called a component of $X$ (some authorities prefer the term connected component). The following facts are easily verified: every non-empty connected subset of $X$ is included in a unique component of $X$; every component of a closed set is closed.

The space $T$ is said to be locally connected if every neighbourhood of any point of $T$ includes a connected neighbourhood of that point (a neighbourhood of a point $p$ is a set $X$ that includes an open set $O$ containing $p$ ). In a locally connected space, every component of an open set is open; note however that components of regular closed sets are closed but, in general, not regular closed, even in locally connected spaces. The space $T$ is said to be unicoherent if, for any closed, connected subsets $X_{1}, X_{2}$ such that $T=X_{1} \cup X_{2}$, the set $X_{1} \cap X_{2}$ is connected. For all $n \geq 1$, the Euclidean space $\mathbb{R}^{n}$ is (obviously) locally connected and (much less obviously) unicoherent 31. A simple example of a non-locally connected space is the rational numbers $\mathbb{Q}$ under the usual metric topology. Simple examples of non-unicoherent spaces are the Jordan curve and the torus. 
The most important properties of local connectedness and unicoherence, from our point of view, are given by the following lemmas.

Lemma 1. Let $X$ be a regular closed subset of a topological space $T$ and $S$ a component of $-X$. If $-X$ has finitely many components, then $\delta S \subseteq X$. Alternatively, if $T$ is locally connected, then $\delta S \subseteq X$.

Proof. For the first statement, let $Z$ be the union of all components of $-X$ other than $S$. By definition, $T=X^{\circ} \cup S \cup Z$. Further, both $S \cap X^{\circ}$ and $S \cap Z$ are empty, whence $T \backslash S=X^{\circ} \cup Z$. Since $X$ is regular closed, and $Z$ is closed (as the union of finitely many closed sets), $(T \backslash S)^{-}=X \cup Z$. Finally, since $S$ is closed, and $S \cap Z=\emptyset, \delta S=S \cap(T \backslash S)^{-} \subseteq X$. For the second statement, suppose, to the contrary, that $\delta S$ contains a point $p$ lying in $(-X)^{\circ}$. Since $S$ is closed, $p \in S$. By local connectedness, let $Y$ be a connected open set such that $p \in Y \subseteq(-X)^{\circ}$. Since $p \in S$ and $Y$ is a connected subset of $-X$, we have $p \in Y \subseteq S$. But this contradicts the assumption that $p \in \delta S$.

Lemma 2. Let $T$ be a unicoherent space and $X \in \mathrm{RC}(T)$ be connected. Then every component of $-X$ has a connected boundary.

Proof. Let $S$ be a connected component of $-X$, and let $Z$ be the union of all components of $-X$ other than $S$. Thus, $T \backslash S=Z \cup X^{\circ}$. We write $S^{*}=(T \backslash S)^{-}$. Since $X$ is regular closed, $S^{*}=Z^{-} \cup X$. By connectedness of $T, X$ intersects every component of $-X$. It follows that $Z \cup X$, and hence $Z^{-} \cup X=S^{*}$ are connected. By definition, $S$ is connected, whence, by unicoherence of $T$, $\delta S=S \cap S^{*}$ is connected.

\subsection{Frames}

A frame is a pair $(T, \mathcal{S})$, where $T$ is a topological space, and $\mathcal{S}$ is a Boolean sub-algebra of $\operatorname{RC}(T)$. Where $T$ is clear from context, we refer to $(T, \mathcal{S})$, simply, as $\mathcal{S}$. Furthermore, where $\mathcal{S}$ is clear from context, we refer to elements of $\mathcal{S}$ as regions. We denote the class of frames of the form $(T, \mathrm{RC}(T))$ by $\mathrm{RC}$. Note that not all frames are of this form: in particular, when working in $n$-dimensional Euclidean spaces, we shall be principally interested in the following proper subalgebra of $\mathrm{RC}\left(\mathbb{R}^{n}\right)$. Any $(n-1)$-dimensional hyperplane bounds two elements of $\mathrm{RC}\left(\mathbb{R}^{n}\right)$ called half-spaces. We denote by $\operatorname{RCP}\left(\mathbb{R}^{n}\right)$ the Boolean sub-algebra of $\mathrm{RC}\left(\mathbb{R}^{n}\right)$ generated by the half-spaces, and call the elements of $\operatorname{RCP}\left(\mathbb{R}^{n}\right)$ (regular closed) polyhedra. If $n=2$, we speak of (regular closed) polygons. Polyhedra may be regarded as 'well-behaved' or, in topologists' parlance, 'tame.' We call $(T, \mathcal{S})$ unicoherent if $T$ is unicoherent, and finitely decomposible if, for all $s \in \mathcal{S}$, there exist connected elements $s_{1}, \ldots, s_{k}$ of $\mathcal{S}$, such that $s=s_{1}+\cdots+s_{k}$. Evidently, $\left(\mathbb{R}^{n}, \operatorname{RCP}\left(\mathbb{R}^{n}\right)\right)$ is finitely decomposible, since any product of halfplanes is connected. Equally obviously:

Lemma 3. Suppose the frame $(T, \mathcal{S})$ is finitely decomposible, and $s \in \mathcal{S}$. Then every component of $s$ is in $\mathcal{S}$, and $s$ is equal to the sum of those components. 
The following basic concepts will be used repeatedly in the sequel. Let $(T, \mathcal{S})$ be a frame. A tuple of elements $\left(s_{0}, \ldots, s_{k-1}\right)$, where $k \geq 1$, will be called a partition, provided

$$
s_{0}+\cdots+s_{k-1}=1 \quad \text { and } \quad s_{i} \cdot s_{j}=0 \text { for all } 0 \leq i<j<k .
$$

We do not insist that the $s_{i}$ are non-empty. We call a partition $\left(s_{0}, \ldots, s_{k-1}\right)$ sub-cyclic if the $s_{i}$ are non-empty and

$$
s_{i} \cap s_{j}=\emptyset, \quad \text { for all } 0 \leq i, j<k \text { such that } 1<j-i<k-1 .
$$

The term 'sub-cyclic' refers to an imagined graph with nodes $\left\{s_{0}, \ldots, s_{k-1}\right\}$ and edges $\left\{\left(s_{i}, s_{j}\right) \mid i \neq j\right.$ and $\left.s_{i} \cap s_{j} \neq \emptyset\right\}$ : this graph is required to be a (not necessarily proper) subgraph of the cyclic graph on $\left\{s_{0}, \ldots, s_{k-1}\right\}$.

Suppose $s$ is a non-empty element of a frame $(T, \mathcal{S})$, and $\vec{s}=\left(s_{0}, \ldots, s_{k-1}\right)$ a partition in that frame. We say that $\vec{s}$ is a colouring of the components of $s$ if every component of $s$ is included in exactly one of the regions of $\vec{s}$. Colourings will be used repeatedly in the sequel, particularly in situations where we may regard the components of $s$ as positions in a finite sequence; by regarding the set of elements of $\vec{s}$ as an alphabet, colourings define words over that alphabet in the obvious way.

\subsection{Topological logics}

In this paper, the focus of attention is not on frames themselves, but rather, on frames as they are described in some language. The languages considered here all employ a countably infinite collection of variables $r_{1}, r_{2}, \ldots$ The language $\mathcal{C}$ is defined by the following syntax:

$$
\begin{aligned}
& \tau::=r\left|\tau_{1}+\tau_{2}\right| \begin{array}{ll|l|l|l}
\tau_{1} \cdot \tau_{2} & -\tau_{1} & 0 & 1,
\end{array} \\
& \varphi \quad::=\tau_{1}=\tau_{2}\left|C\left(\tau_{1}, \tau_{2}\right) \quad\right| \quad \varphi_{1} \vee \varphi_{2}\left|\varphi_{1} \wedge \varphi_{2}\right| \neg \varphi_{1} .
\end{aligned}
$$

The language $\mathcal{B}$ is defined analogously, but without the predicate $C$. Thus, $\mathcal{B}$ is the quantifier-free language of the variety of Boolean algebras.

An interpretation over a frame $(T, \mathcal{S})$ is a function.$^{\mathfrak{I}}$ mapping variables $r$ to elements $r^{\mathfrak{I}}$ of $\mathcal{S}$. We extend.$^{\mathfrak{I}}$ to terms $\tau$ by setting $\left(\tau_{1}+\tau_{2}\right)^{\mathfrak{I}}=\tau_{1}^{\mathfrak{I}}+\tau_{2}^{\mathfrak{I}}$, $\left(\tau_{1} \cdot \tau_{2}\right)^{\mathfrak{I}}=\tau_{1}^{\mathfrak{I}} \cdot \tau_{2}^{\mathfrak{I}},\left(-\tau_{1}\right)^{\mathfrak{I}}=-\left(\tau_{1}^{\mathfrak{I}}\right), 0^{\mathfrak{I}}=\emptyset$ and $1^{\mathfrak{I}}=T$. We write $\mathfrak{I}=\tau_{1}=\tau_{2}$ if and only if $\tau_{1}^{\mathfrak{I}}=\tau_{2}^{\mathfrak{I}}$, and $\mathfrak{I} \models C\left(\tau_{1}, \tau_{2}\right)$ if and only if $\tau_{1}^{\mathfrak{I}} \cap \tau_{2}^{\mathfrak{I}} \neq \emptyset$, extending this relation to non-atomic formulas in the standard way. We read $C\left(\tau_{1}, \tau_{2}\right)$ as ' $\tau_{1}$ contacts $\tau_{2}$.' If $\varphi$ is a formula whose variables, taken in some order, are $\vec{r}=\left(r_{1}, \ldots, r_{n}\right)$, and $\mathfrak{I} \models \varphi$, then the tuple $\vec{a}=\left(r_{1}^{\mathfrak{J}}, \ldots, r_{n}^{\mathfrak{I}}\right)$ is said to satisfy $\varphi(\vec{r})$; in such a case, we will often say ' $\vec{a}$ satisfies $\varphi(\vec{r})$.'

We remark that the property that a $k$-tuple $\left(r_{0}, \ldots, r_{k-1}\right)$ forms a partition is evidently expressible using the $\mathcal{B}$-formula

$$
\operatorname{part}\left(r_{0}, \ldots, r_{k-1}\right)=\left(\sum_{i=0}^{k-1} r_{i}=1\right) \wedge \bigwedge_{0 \leq i<j<k}\left(r_{i} \cdot r_{j}=0\right) .
$$


The property that a $k$-tuple forms a sub-cyclic partition is expressible using the $\mathcal{C}$-formula

$$
\begin{aligned}
& \text { sc-part }\left(r_{0}, \ldots, r_{k-1}\right)= \\
& \operatorname{part}\left(r_{0}, \ldots, r_{k-1}\right) \wedge \bigwedge_{0 \leq i<k}\left(r_{i} \neq 0\right) \wedge \bigwedge_{1<j-i<k-1} \neg C\left(r_{i}, r_{j}\right) .
\end{aligned}
$$

And, assuming that $\operatorname{part}\left(r_{0}, \ldots, r_{k-1}\right)$ is satisfied, the $\mathcal{C}$-formula

$$
\operatorname{colour}\left(r ; r_{0}, \ldots, r_{k-1}\right)=\bigwedge_{0 \leq i<j<k} \neg C\left(\left(r \cdot r_{i}\right),\left(r \cdot r_{j}\right)\right)
$$

ensures that the partition $r_{0}, \ldots, r_{k-1}$ colours the components of $r$. Conversely, over finitely decomposible frames, any colouring of $r$ by a partition $r_{0}, \ldots, r_{k-1}$ must satisfy colour $\left(r ; r_{0}, \ldots, r_{k-1}\right)$.

Turning to connectedness predicates, we define the languages $\mathcal{B} c$ and $\mathcal{C} c$ to be extensions of $\mathcal{B}$ and $\mathcal{C}$, respectively, with the unary predicate $c$. We set $\mathfrak{I}=c(\tau)$ if and only if $\tau^{\mathfrak{I}}$ is connected in the topological space under consideration. Similarly, we define $\mathcal{B} c^{\circ}$ and $\mathcal{C} c^{\circ}$ to be extensions of $\mathcal{B}$ and $\mathcal{C}$ with the predicate $c^{\circ}$, setting $\mathfrak{I}=c^{\circ}(\tau)$ if and only if $\left(\tau^{\mathfrak{I}}\right)^{\circ}$ is connected. If $\mathcal{K}$ is a class of frames, and $\mathcal{L}$ is one of $\mathcal{B} c, \mathcal{C} c, \mathcal{B} c^{\circ}$ or $\mathcal{C} c^{\circ}$, then $\operatorname{Sat}(\mathcal{L}, \mathcal{K})$ is the set of $\mathcal{L}$-formulas satisfiable over $\mathcal{K}$.

Setting $\mathcal{K}=\mathrm{RC}$, the complexity of this problem in known for all of the languages $\mathcal{L}$ considered above [27, 28. If $\varphi$ is a formula of any of the languages $\mathcal{B} c, \mathcal{C} c$ or $\mathcal{C} c^{\circ}$, and $\varphi$ is satisfiable over RC, then $\varphi$ is satisfiable over some frame $\operatorname{RC}(T)$, where $|T|$ is bounded by a singly-exponential function of $|\varphi|$; and the problems $\operatorname{Sat}(\mathcal{L}, \mathrm{RC})$, for $\mathcal{L} \in\left\{\mathcal{B} c, \mathcal{C} c, \mathcal{C} c^{\circ}\right\}$, are all ExpTime-complete. On the other hand, if $\psi$ is a $\mathcal{B} c^{\circ}$-formula satisfiable over $\mathrm{RC}$, then $\psi$ is satisfiable over some frame $\operatorname{RC}(T)$, where $|T|$ is bounded by a polynomial function of $|\psi|$; and the problem $\operatorname{Sat}\left(\mathcal{B} c^{\circ}, \mathrm{RC}\right)$ is NP-complete. Thus, we observe a difference between $\mathcal{B} c, \mathcal{C} c$ and $\mathcal{C} c^{\circ}$ on the one hand, and $\mathcal{B} c^{\circ}$ on the other.

However, satisfiability over RC is of little interest from the point of view of Artificial Intelligence, where almost all conceivable applications concern the frames over Euclidean space of dimensions 2 or 3 . Accordingly, we shall be concerned with $\operatorname{Sat}(\mathcal{L}, \mathcal{K})$, where $\mathcal{L}$ is any of $\mathcal{B} c, \mathcal{B} c^{\circ}, \mathcal{C} c$ or $\mathcal{C} c^{\circ}$, and $\mathcal{K}$ is $\left\{\mathrm{RC}\left(\mathbb{R}^{n}\right)\right\}$ or $\left\{\operatorname{RCP}\left(\mathbb{R}^{n}\right)\right\}$ for $n \geq 2$. For ease of reading, we write $\operatorname{Sat}\left(\mathcal{L}, \operatorname{RC}\left(\mathbb{R}^{n}\right)\right)$ and $\operatorname{Sat}\left(\mathcal{L}, \operatorname{RCP}\left(\mathbb{R}^{n}\right)\right)$ rather than $\operatorname{Sat}\left(\mathcal{L},\left\{\operatorname{RC}\left(\mathbb{R}^{n}\right)\right\}\right)$ and $\operatorname{Sat}\left(\mathcal{L},\left\{\operatorname{RCP}\left(\mathbb{R}^{n}\right)\right\}\right)$.

\subsection{Graphs}

Unless explicitly indicated to the contrary, all graphs in this paper are taken to be finite, and to have no multiple edges and no loops: i.e., if $G=(V, E)$ is a graph, $\left(v, v^{\prime}\right) \in E$ implies $v \neq v^{\prime}$. We also assume that the edges have no direction, i.e., $\left(v, v^{\prime}\right) \in E$ if and only if $\left(v^{\prime}, v\right) \in E$. A path in $G$ is a sequence of distinct vertices $v_{0}, \ldots, v_{n-1}$ such that $\left(v_{i}, v_{i+1}\right)$ is an edge, for all $0 \leq i<n-1$; further, a cycle in $G$ is a path $v_{0}, \ldots, v_{n-1}$ such that, in addition, $\left(v_{n-1}, v_{0}\right)$ is 
an edge. Informally, in this case, we speak of the sequence $v_{0}, \ldots, v_{n-1}, v_{0}$ as a cycle. A graph is connected if any two nodes are joined by some path; a graph which contains no cycles is acyclic; and a connected, acyclic graph is a tree. If $G$ is a tree, then any pair of nodes in $G$ is joined by a unique path. Further, if $v_{0}, \ldots, v_{n-1}$ is a sequence of nodes in $G$ such that $\left(v_{i}, v_{i+1}\right)$ is an edge for all $0 \leq i<n-1$, and $v_{i} \neq v_{i+2}$ for all $0 \leq i<n-2$, then this sequence contains no duplicates, and thus is a path.

Let $\mathcal{S}$ be a finitely decomposible frame over some topological space, and $\vec{s}$ a connected partition in $\mathcal{S}$. We can associate a graph with $\vec{s}$, denoted $H(\vec{s})$, as follows: the vertices of $H(\vec{s})$ are the components of the elements of $\vec{s}$; the edges of $H(\vec{s})$ are the pairs $(X, Y)$ such that $X \neq Y$ and $X \cap Y \neq \emptyset$. We refer to $H(\vec{s})$ as the component graph of $\vec{s}$. Note that the number of vertices of $H(\vec{s})$ is in general larger than the number of elements in $\vec{s}$; however, since $\mathcal{S}$ is finitely decomposible, this number is still finite.

We prove a simple but powerful lemma connecting some of the notions encountered above.

Lemma 4. Let $T$ be a unicoherent topological space, $\mathcal{S}$ a finitely decomposible frame on $T$, and $\vec{s}$ a sub-cyclic partition in $\mathcal{S}$. Then the component graph, $H(\vec{s})$, is a tree.

Proof. Write $\vec{s}=\left(s_{0}, \ldots, s_{n-1}\right)$. Since $\mathcal{S}$ is finitely decomposible, and $T$ is connected, $H(\vec{s})$ is obviously finite and connected. We need only show that it contains no cycles. If $n=1$, then $|H(\vec{s})|=1$, and this is trivial. We assume, for ease of formulation, that $n \geq 4$, since a similar (and in fact simpler) argument applies if $n=2$ or $n=3$.

Suppose $\left(X_{0}, X_{1}\right)$ is an edge of $H(\vec{s})$. We may assume, without loss of generality, that $X_{0}$ is a component of $s_{0}$, and $X_{1}$ a component of $s_{1}$. The subcyclicity condition ensures that $s_{0} \cap s_{i}=\emptyset$ for $2 \leq i<n-1$, and $s_{1} \cap s_{i}=\emptyset$ for $3 \leq i<n$. Now let $S$ be the component of $-X_{1}$ containing $X_{0}$ : we claim that $\delta S \subseteq s_{0}$. By the first statement of Lemma 1, $\delta S \subseteq X_{1} \subseteq s_{1}$, whence $\delta S$ contains no point of $s_{3}+\cdots+s_{n-1}$. On the other hand, $\delta S$ is obviously included in $-s_{1}=s_{0}+s_{2}+s_{3}+\cdots s_{n-1}$, and hence in $s_{0}+s_{2}$. Since $s_{0} \cap s_{2}=\emptyset$, and, by Lemma 2, $\delta S$ is connected, we have either $\delta S \subseteq s_{0}$ or $\delta S \subseteq s_{2}$. Now, since $\left(X_{0}, X_{1}\right)$ is an edge of $H(\vec{s})$, and any point of $X_{0} \cap X_{1}$ must lie in both $S$ and $-S$, we have $\delta S \cap X_{0} \neq \emptyset$, and, therefore, $\delta S \subseteq s_{0}$, as claimed.

Now suppose $\left(X_{1}, X_{2}\right)$ is also an edge of $H(\vec{s})$, with $X_{0}$ and $X_{2}$ distinct. We claim that $X_{0}$ and $X_{2}$ lie in different components of $-X_{1}$ (i.e., $X_{2} \nsubseteq S$ ). For suppose otherwise. Again, since any point of $X_{1} \cap X_{2}$ lies in both $S$ and $-S, \delta S \cap X_{2} \neq \emptyset$. Furthermore, since $s_{1} \cap s_{i}=\emptyset$ for $3 \leq i<n, X_{2}$ must be a component of either $s_{0}$ or $s_{2}$. But if $X_{2} \subseteq s_{0}$, then the connected set $\delta S \subseteq s_{0}$ has points in common with the components $X_{0}, X_{2}$ of $s_{0}$, contradicting the assumption that $X_{0}$ and $X_{2}$ are distinct. On the other hand, if $X_{2} \subseteq s_{2}$ then $\delta S \subseteq s_{0}$ contains a point of $s_{2}$, which is again impossible.

Finally, suppose that $X_{0}, X_{1}, X_{2}, \ldots, X_{m}$ is a cycle in $H(\vec{s})$, where $m \geq 3$ and $X_{m}=X_{0}$. Then the connected set $X_{2}+\cdots+X_{m-1}+X_{0}$ lies entirely 
in $-X_{1}$, contradicting the fact that $X_{0}$ and $X_{2}$ lie in different components of $-X_{1}$.

\subsection{Post correspondence problem}

In the sequel, we make use of the well-known Post correspondence problem (PCP). Fix finite alphabets $T$ and $U$, where $|T| \geq 7$ and $|U| \geq 2$. A morphism from $T$ to $U$ is a function $\mathrm{w}: T \rightarrow U^{*}$ mapping each element of $T$ to a word over $U$. We extend $\mathrm{w}$ to a mapping $\mathrm{w}: T^{*} \rightarrow U^{*}$ by defining, for any word $\tau=t_{1} \cdots t_{k} \in T^{*}, \mathrm{w}(\tau)=\mathrm{w}\left(t_{1}\right) \cdots \mathrm{w}\left(t_{k}\right)$. An instance of the PCP is a pair of morphisms $\mathrm{W}=\left(\mathrm{w}^{1}, \mathrm{w}^{2}\right)$ from $T$ to $U$. The instance $\mathrm{W}$ is positive if there exists a non-empty word $\tau \in T^{*}$ such that $\mathrm{w}^{1}(\tau)=\mathrm{w}^{2}(\tau)$. Intuitively, we are invited to think of each element of $T$ as a 'tile' inscribed with an 'upper' word over $U$, given by $\mathrm{w}^{1}(t)$, and a 'lower' word over $U$, given by $\mathrm{w}^{2}(t)$; we are asked to determine, for the given collection of tiles, whether there exists a non-empty, finite sequence of these tiles (repeats allowed) such that the concatenation of their upper words equals the concatenation of their lower words. The set of positive PCP instances is known to be r.e.-complete [37, and remains so even under the restriction that $\mathrm{w}^{k}(t)$ is non-empty for every $t \in T$. In fact, nothing hinges on the exact choice of $T$ and $U$, subject to the restrictions mentioned above. In particular, we may assume $T$ and $U$ are disjoint.

\section{Forcing infinitely many components in locally connected unicoherent spaces.}

In this section, we construct $\mathcal{C} c$ -, $\mathcal{C} c^{\circ}$ - and $\mathcal{B} c$-formulas $\varphi$ with the following properties: (i) $\varphi$ is satisfiable over $\mathrm{RC}\left(\mathbb{R}^{n}\right)$ for all $n \geq 2$; (ii) if $T$ is a locally connected, unicoherent space and $\vec{a}$ is a tuple from $\operatorname{RC}(T)$ satisfying $\varphi$, then $\vec{a}$ includes members with infinitely many connected components. Since $\operatorname{RCP}\left(\mathbb{R}^{n}\right)$ is finitely decomposible, these properties entail that $\operatorname{Sat}\left(\mathcal{L}, \operatorname{RC}\left(\mathbb{R}^{n}\right)\right) \neq \operatorname{Sat}\left(\mathcal{L}, \operatorname{RCP}\left(\mathbb{R}^{n}\right)\right)$ for $\mathcal{L}$ any of $\mathcal{C} c, \mathcal{C} c^{\circ}$ or $\mathcal{B} c$, and all $n \geq 2$. Furthermore, the techniques developed in this section will be used in Sec. 4 to prove that satisfiability of $\mathcal{C} c-, \mathcal{C} c^{\circ}$ - and $\mathcal{B} c$-formulas over $\operatorname{RCP}\left(\mathbb{R}^{n}\right)$, for $n \geq 2$, is undecidable.

We now construct a $\mathcal{C} c$-formula, $\varphi_{\infty}$ with properties $(i)$ and $(i i)$. As an aid to intuition, consider any locally connected unicoherent space $T$. We equivocate between variables and the regions they are assigned in some putative interpretation over $\mathrm{RC}(T)$. In this section we write $\lfloor i\rfloor$ to denote the value of $i$ modulo 4. The first conjunct of $\varphi_{\infty}$ states that $r_{0}, r_{1}, r_{2}, r_{3}$ form a sub-cyclic partition:

$$
\text { sc-part }\left(r_{0}, r_{1}, r_{2}, r_{3}\right) \text {. }
$$

We also require non-empty sub-regions $r_{i}^{\prime}$ of $r_{i}$ and a non-empty region $t$ :

$$
\bigwedge_{i=0}^{3}\left(\left(r_{i}^{\prime} \neq 0\right) \wedge\left(r_{i}^{\prime} \leq r_{i}\right)\right),
$$




$$
(t \neq 0)
$$

The configuration we have in mind is depicted in Fig. 3. where components of the $r_{i}$ are arranged like the layers of an onion. The 'innermost' component

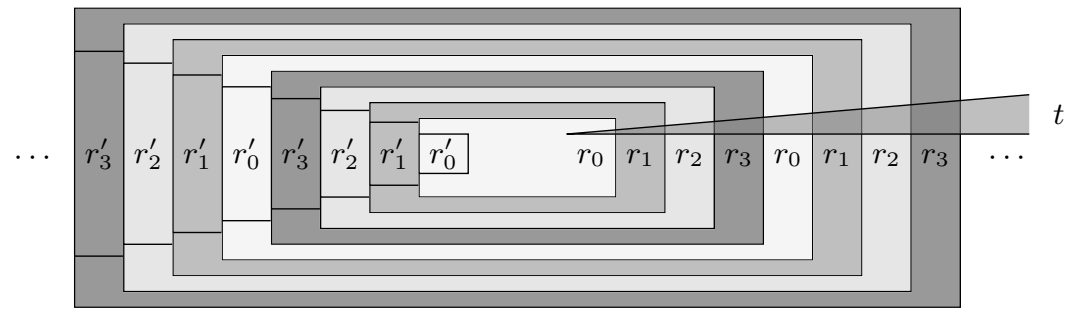

Figure 3: Regions satisfying $\varphi_{\infty}$.

of $r_{0}$ is surrounded by a component of $r_{1}$, which in turn is surrounded by a component of $r_{2}$, and so on. The region $t$ passes through every layer, but avoids the $r_{i}^{\prime}$. To enforce a configuration of this sort, we need the following formulas:

$$
\begin{aligned}
& \bigwedge_{i=0}^{3} c\left(r_{i}^{\prime}+r_{\lfloor i+1\rfloor}+t\right), \\
& \bigwedge_{i=0}^{3} \neg C\left(r_{i}^{\prime}, t\right), \\
& \bigwedge_{i=0}^{3} \neg C\left(r_{i}^{\prime}, r_{\lfloor i+1\rfloor} \cdot\left(-r_{\lfloor i+1\rfloor}^{\prime}\right)\right) .
\end{aligned}
$$

Observe that (7)-9 ensure each component of $r_{i}^{\prime}$ is in contact with $r_{\lfloor i+1\rfloor}^{\prime}$. Denote by $\varphi_{\infty}$ the conjunction of (4)-(9).

Theorem 5. The $\mathcal{C} c$-formula $\varphi_{\infty}$ is satisfiable over $\mathrm{RC}\left(\mathbb{R}^{n}\right), n \geq 2$. On the other hand, if $T$ is a locally connected, unicoherent space, then any tuple from $\mathrm{RC}(T)$ satisfying $\varphi_{\infty}$ features sets that have infinitely many components.

Proof. Fig. 3 shows how $\varphi_{\infty}$ can be satisfied over $\mathrm{RC}\left(\mathbb{R}^{2}\right)$. By cylindrification, it is also satisfiable over any $\operatorname{RC}\left(\mathbb{R}^{n}\right)$, for $n>2$. This establishes the first statement of the lemma. For the second statement, we suppose that $\varphi_{\infty}$ is satisfied in a frame on a locally connected, unicoherent space $T$; we show that some members of the satisfying tuple have infinitely many components. To avoid clumsy circumlocutions, we equivocate between variables and the regions to which they are assigned in the satisfying interpretation: thus we speak about the 'regions' $r_{0}, \ldots, r_{3}, r_{0}^{\prime}, \ldots, r_{3}^{\prime}$ and $t$. No confusion should result.

We proceed by constructing a sequence of disjoint components $X_{i}$ of $r_{\lfloor i\rfloor}$ and open sets $V_{i}$ connecting $X_{i}$ to $X_{i+1}$; see Fig. 4. By the first conjunct of (5), let $X_{0}$ be a component of $r_{0}$ containing points in $r_{0}^{\prime}$. Suppose $X_{i}$ has been 


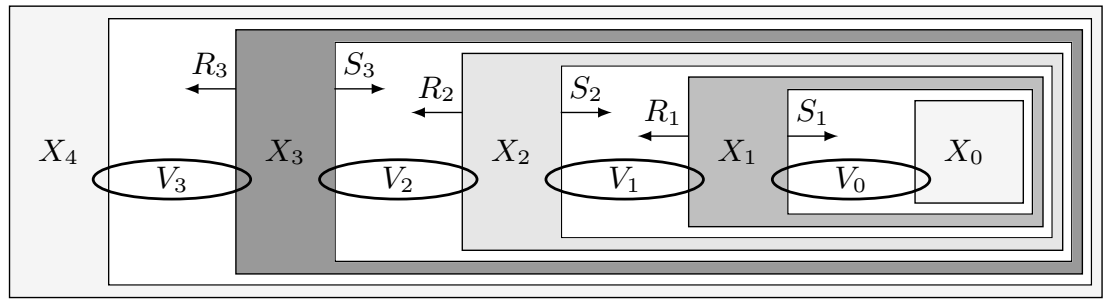

Figure 4: Sequences $X_{0}, X_{1}, \ldots$ of components $X_{i}$ of $r_{\lfloor i\rfloor}$ and $V_{0}, V_{1}, \ldots$ of open sets $V_{i}$ connecting $X_{i}$ to $X_{i+1}$ with the 'holes' $S_{i+1}$ and $R_{i+1}$ of $X_{i+1}$ containing $X_{i}$ and $X_{i+2}$, respectively.

constructed. By (7)-(9), $X_{i}$ is in contact with $r_{\lfloor i+1\rfloor}^{\prime}$. Using (4) and the fact that $T$ is locally connected, one can find a component $X_{i+1}$ of $r_{\lfloor i+1\rfloor}$ which has points in $r_{\lfloor i+1\rfloor}^{\prime}$, and a connected open set $V_{i}$ such that $V_{i} \cap X_{i}$ and $V_{i} \cap X_{i+1}$ are non-empty, but $V_{i} \cap r_{\lfloor i+2\rfloor}$ is empty.

To see that the $X_{i}$ are distinct, let $S_{i+1}$ and $R_{i+1}$ be the components of $-X_{i+1}$ containing $X_{i}$ and $X_{i+2}$, respectively. It suffices to show that we have $S_{i+1} \subseteq S_{i+2}^{\circ}$. Note that the connected set $V_{i}$ must intersect $\delta S_{i+1}$. By the second statement of Lemma 1. $\delta S_{i+1} \subseteq X_{i+1} \subseteq r_{\lfloor i+1\rfloor}$. Also, $\delta S_{i+1} \subseteq-X_{i+1}$; hence, by (4), $\delta S_{i+1} \subseteq r_{\lfloor i\rfloor} \cup r_{\lfloor i+2\rfloor}$. By Lemma 2, $\delta S_{i+1}$ is connected, and therefore, by (4), $\delta S_{i+1}$ is entirely contained either in $r_{\lfloor i\rfloor}$ or in $r_{\lfloor i+2\rfloor}$. Since $V_{i} \cap \delta S_{i+1} \neq \emptyset$ and $V_{i} \cap r_{\lfloor i+2\rfloor}=\emptyset$, we have $\delta S_{i+1} \nsubseteq r_{\lfloor i+2\rfloor}$, so $\delta S_{i+1} \subseteq r_{\lfloor i\rfloor}$. Similarly, $\delta R_{i+1} \subseteq r_{\lfloor i+2\rfloor}$. By (4), then, $\delta S_{i+1} \cap \delta R_{i+1}=\emptyset$, and since $S_{i+1}$ and $R_{i+1}$ are components of the same set, and have non-empty boundaries, they are disjoint. Hence, we obtain $S_{i+1} \subseteq\left(-R_{i+1}\right)^{\circ}$, and since $X_{i+2} \subseteq R_{i+1}$, also $S_{i+1} \subseteq\left(-X_{i+2}\right)^{\circ}$. So, using local connectedness again, $S_{i+1}$ lies in the interior of a component of $-X_{i+2}$, and since $\delta S_{i+1} \subseteq X_{i+1} \subseteq S_{i+2}$, that component must be $S_{i+2}$.

Now we show how the $\mathcal{C} c$-formula $\varphi_{\infty}$ can be transformed to $\mathcal{C} c^{\circ}$ - and $\mathcal{B} c$ formulas with similar properties. Note first that all occurrences of $c$ in $\varphi_{\infty}$ have positive polarity. Let $\varphi_{\infty}^{\circ}$ be the result of replacing them with the predicate $c^{\circ}$. In Fig. 3, the connected regions mentioned in (7) are in fact interior-connected; hence $\varphi_{\infty}^{\circ}$ is satisfiable over $\mathrm{RC}\left(\mathbb{R}^{n}\right)$. Since interior-connectedness implies connectedness, $\varphi_{\infty}^{\circ}$ entails $\varphi_{\infty}$, and we obtain:

Corollary 6. The $\mathcal{C} c^{\circ}$-formula $\varphi_{\infty}^{\circ}$ is satisfiable over $\mathrm{RC}\left(\mathbb{R}^{n}\right), n \geq 2$. On the other hand, if $T$ is a locally connected, unicoherent space, then any tuple from $\mathrm{RC}(T)$ satisfying $\varphi_{\infty}^{\circ}$ features sets that have infinitely many components.

We next consider the language $\mathcal{B} c$. Observe that all occurrences of $C$ in $\varphi_{\infty}$ are negative. We eliminate these using the predicate $c$ : we use the fact that, if the sum of two connected regions is not connected, then they are not in contact. If $\tau_{1}$ and $\tau_{2}$ are any terms, we employ the abbreviation

$$
\operatorname{notC}\left(\tau_{1}, \tau_{2}\right)=c\left(\tau_{1}\right) \wedge c\left(\tau_{2}\right) \wedge \neg c\left(\tau_{1}+\tau_{2}\right) .
$$


Observe that $\operatorname{not} \mathrm{C}\left(\tau_{1}, \tau_{2}\right)$ is always a $\mathcal{B} c$-formula. Furthermore, $\operatorname{not} \mathrm{C}\left(\tau_{1}, \tau_{2}\right)$ implies $\neg C\left(\tau_{1}^{\prime}, \tau_{2}^{\prime}\right)$ for any $\tau_{1}^{\prime} \leq \tau_{1}$ and $\tau_{2}^{\prime} \leq \tau_{2}$. Now we replace (8) by

$$
\operatorname{not} \mathrm{C}\left(r_{0}^{\prime}+r_{1}^{\prime}+r_{2}^{\prime}+r_{3}^{\prime}, t\right) \text {. }
$$

The resulting formula thus implies the original; on the other hand, it is satisfied by the configuration of Fig. 3. Next, we replace each conjunct $\neg C\left(r_{i}^{\prime}, r_{\lfloor i+1\rfloor}\right.$. $\left.\left(-r_{\lfloor i+1\rfloor}^{\prime}\right)\right)$ in $(9)$ by

$$
\operatorname{notC}\left(r_{i}^{\prime}+s, \quad r_{\lfloor i+1\rfloor} \cdot\left(-r_{\lfloor i+1\rfloor}^{\prime}\right)+t\right),
$$

where $s$ is a fresh variable. Again, the resulting formula implies the original, and, furthermore, is evidently satisfied by the configuration of Fig. 5, where $s$ lies inside $\sum_{j=0}^{3} r_{j}^{\prime}$, symmetrically to $t$ lying inside $\sum_{j=0}^{3}\left(r_{j} \cdot\left(-r_{j}^{\prime}\right)\right)$. The

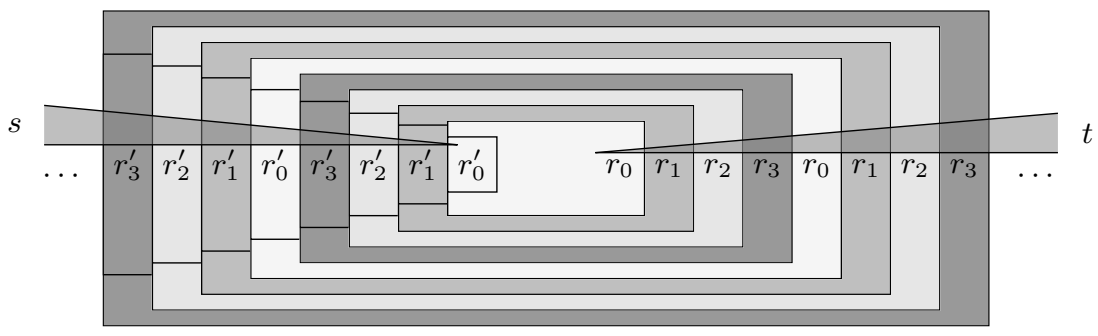

Figure 5: Region $s$ lying inside $\sum_{j=0}^{3} r_{j}^{\prime}$ and connecting the components of each $r_{i}^{\prime}$.

only remaining occurrences of the contact predicate $C$ are in (4). We deal with them by partitioning the regions: instead of each $\neg C\left(r_{i}, r_{\lfloor i+2\rfloor}\right)$ we consider the equivalent conjunction of 4 formulas:

$$
\begin{array}{llll}
\neg C\left(r_{i}^{\prime}, r_{\lfloor i+2\rfloor}^{\prime}\right) & \wedge & \neg C\left(r_{i} \cdot\left(-r_{i}^{\prime}\right), \quad r_{\lfloor i+2\rfloor} \cdot\left(-r_{\lfloor i+2\rfloor}^{\prime}\right)\right) \wedge \\
\neg C\left(r_{i} \cdot\left(-r_{i}^{\prime}\right),\right. & \left.r_{\lfloor i+2\rfloor}^{\prime}\right) & \wedge & \neg C\left(r_{i}^{\prime}, \quad r_{\lfloor i+2\rfloor} \cdot\left(-r_{\lfloor i+2\rfloor}^{\prime}\right)\right) .
\end{array}
$$

The formulas in the second row are replaced by

$$
\operatorname{notC}\left(r_{i} \cdot\left(-r_{i}^{\prime}\right)+t, \quad r_{\lfloor i+2\rfloor}^{\prime}+s\right) \wedge \operatorname{notC}\left(r_{i}^{\prime}+s, \quad r_{\lfloor i+2\rfloor} \cdot\left(-r_{\lfloor i+2\rfloor}^{\prime}\right)+t\right) .
$$

Again, the resulting formula implies the original and is satisfied by the configuration of Fig. 5 . The formulas in the first row are replaced by

$$
\operatorname{not} \mathrm{C}\left(r_{i}^{\prime}+s_{i}, \quad r_{\lfloor i+2\rfloor}^{\prime}+s_{\lfloor i+2\rfloor}\right) \wedge \operatorname{notC}\left(r_{i} \cdot\left(-r_{i}^{\prime}\right)+t_{i}, \quad r_{\lfloor i+2\rfloor} \cdot\left(-r_{\lfloor i+2\rfloor}^{\prime}\right)+t_{\lfloor i+2\rfloor}\right) .
$$

Again, the resulting formula implies the original. To see that it is still satisfiable, we select regions $s_{0}, \ldots, s_{3}$, with $s_{i}$ and $s_{i+2}$ disjoint $(i=0,1)$, such that each $s_{i}(0 \leq i<4)$ connects together the components of $r_{i}^{\prime}$ as shown in Fig. 6. In a symmetric way, select regions $t_{0}, \ldots, t_{3}$, with $t_{i}$ and $t_{i+2}$ disjoint $(i=0,1)$, 


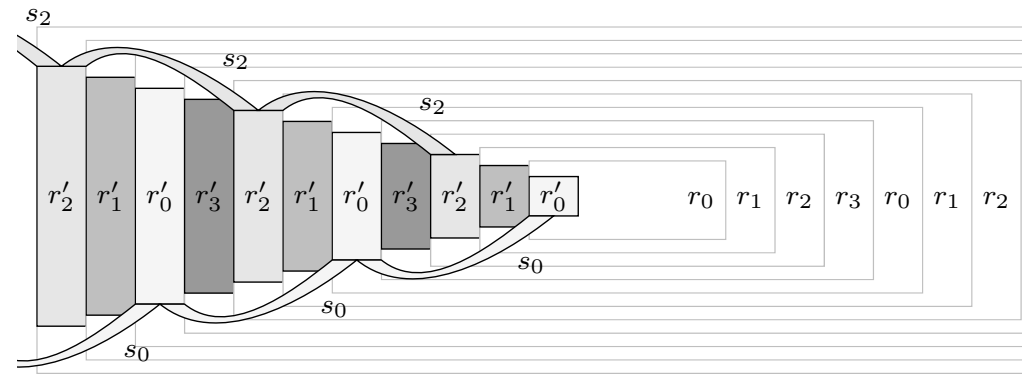

Figure 6: Disjoint connected regions $s_{0}$ and $s_{2}$ containing the components of $r_{0}^{\prime}$ and $r_{2}^{\prime}$, respectively.

such that each $t_{i}(0 \leq i<4)$ connects together the components of $r_{i} \cdot\left(-r_{i}^{\prime}\right)$. Transforming $\varphi_{\infty}$ in the way just described, we obtain a $\mathcal{B} c$-formula $\varphi_{\infty}^{c}$ with the required properties.

Theorem 7. The $\mathcal{B} c$-formula $\varphi_{\infty}^{c}$ is satisfiable over $\mathrm{RC}\left(\mathbb{R}^{n}\right), n \geq 2$. On the other hand, if $T$ is a locally connected, unicoherent space, then any tuple from $\mathrm{RC}(T)$ satisfying $\varphi_{\infty}^{c}$ features sets that have infinitely many components.

The results of this section make no reference to the language $\mathcal{B} c^{\circ}$. In fact, an analogue of Theorem 7 for $\mathcal{B} c^{\circ}$ will be proved in the special case $n=2$, in Sec. 5.1. using a planarity argument. For $n \geq 3$, however, this result fails, as we show in Sec. 6. As we observed above, Theorem 7 shows that, for all $n \geq 2$, $\operatorname{Sat}\left(\mathcal{B} c, \mathrm{RC}\left(\mathbb{R}^{n}\right)\right) \neq \operatorname{Sat}\left(\mathcal{B} c, \mathrm{RCP}\left(\mathbb{R}^{n}\right)\right)$. The reader will recall from Sec. 1.3 that the corresponding inequations for the language $\mathcal{B} c^{\circ}$ hold anyway, by (3). Finally, we remark on the case of the real line, $\mathbb{R}$, which was considered in 28 . The analogue of Theorem 5 for the case $n=1$ holds (though we need to use a different formula to force an infinitude of components); however, the analogue of Theorem 7 for $n=1$ fails: indeed, we have $\operatorname{Sat}(\mathcal{B} c, \mathrm{RC}(\mathbb{R}))=\operatorname{Sat}(\mathcal{B} c, \mathrm{RCP}(\mathbb{R}))$.

\section{Undecidability: the polyhedral case}

We use the techniques developed in the previous section to prove that the satisfiability problem for any of the languages $\mathcal{B} c, \mathcal{C} c$ or $\mathcal{C} c^{\circ}$ over the frame $\operatorname{RCP}\left(\mathbb{R}^{n}\right)$, $n \geq 2$, is undecidable. Recall that a frame $(T, \mathcal{S})$ is unicoherent if $T$ is unicoherent; and $(T, \mathcal{S})$ is finitely decomposible if, for all $s \in \mathcal{S}$, there exist connected elements $s_{1}, \ldots, s_{k}$ of $\mathcal{S}$, such that $s=s_{1}+\cdots+s_{k}$.

Theorem 8. Let $\mathcal{K}$ be any class of unicoherent, finitely decomposible frames, such that $\mathcal{K}$ contains some frame of the form $\left(\mathbb{R}^{n}, \mathcal{S}\right), n \geq 2$, where $\operatorname{RCP}\left(\mathbb{R}^{n}\right) \subseteq$ $\mathcal{S}$. Then the problem $\operatorname{Sat}(\mathcal{C} c, \mathcal{K})$ is r.e.-hard. 
Proof. We proceed via a reduction of the Post correspondence problem (PCP), constructing, for any instance $\mathrm{W}$, a formula $\varphi_{\mathrm{W}}$ with the property that $(i)$ if $\mathrm{W}$ is positive then $\varphi_{\mathrm{W}}$ is satisfiable over $\operatorname{RCP}\left(\mathbb{R}^{n}\right), n \geq 2$, and (ii) if $\varphi_{\mathrm{W}}$ is satisfiable over a unicoherent, finitely decomposible frame then $\mathrm{W}$ is positive. The formula $\varphi_{\mathrm{W}}$ will be a conjunction of $\mathcal{C} c$-literals. As in the proof of Theorem 5 , we equivocate between variables and the regions to which they are assigned in some putative interpretation over a frame in $\mathcal{K}$ : this will allow us to motivate the conjuncts of $\varphi_{\mathrm{W}}$ as they are presented. In the remainder of this proof, if $i$ is an integer, $\lfloor i\rfloor$ indicates the value of $i$ modulo 4 .

Let the PCP-instance $\mathrm{W}=\left(\mathrm{w}^{1}, \mathrm{w}^{2}\right)$ over alphabets $T$ and $U$ be given, and let $\vec{r}=\left(r_{0}, \ldots, r_{3}\right)$ and $\vec{s}=\left(s_{0}, \ldots, s_{3}\right)$ be tuples of variables. The first conjuncts of $\varphi_{\mathrm{W}}$ ensure that $\vec{r}$ and $\vec{s}$ are sub-cyclic partitions:

$$
\begin{aligned}
& \text { sc-part }\left(r_{0}, r_{1}, r_{2}, r_{3}\right), \\
& \text { sc-part }\left(s_{0}, s_{1}, s_{2}, s_{3}\right) .
\end{aligned}
$$

By Lemma 4, the component graphs $H(\vec{r})$ and $H(\vec{s})$ are trees. Thus, any two vertices of $H(\vec{r})$ are joined by a unique path, and likewise for $H(\vec{s})$. The vertices of $H(\vec{r})$ will be used to represent letters in some word $v \in U^{*}$, and those of $H(\vec{s})$, letters in some word $\tau \in T^{*}$.

Let $e^{1}$ and $e^{2}$ be fresh variables. We shall use these to represent the morphisms $w^{1}$ and $w^{2}$, respectively. The next conjuncts of $\varphi w$ ensure that, for all $0 \leq i<4$, the components of both $r_{i} \cdot e^{1}$ and $r_{i} \cdot e^{2}$ are coloured by the elements $\vec{s}$, as defined in Sec. 2 .

$$
\bigwedge_{k=1}^{2} \bigwedge_{i=0}^{3} \operatorname{colour}\left(r_{i} \cdot e^{k} ; s_{0}, s_{1}, s_{2}, s_{3}\right) .
$$

Fig. 7 shows a configuration conforming to these conditions. In this arrangement, $H(\vec{r})$ has vertices $\left\{A_{1}, \ldots, A_{7}\right\}$, where $A_{i}$ is a component of $r_{\lfloor i\rfloor}$, and $H(\vec{s})$ has vertices $\left\{B_{1}, \ldots, B_{4}\right\}$ (indicated by thick boundaries), where $B_{j}$ is a component of $s_{\lfloor j\rfloor}$. Observe that, for $0 \leq i<4$ and $1 \leq k \leq 2$, each component of $r_{i} \cdot e^{k}$ is included in exactly one of $s_{0}, \ldots, s_{3}$, and hence in a single vertex $B_{j}$ of $H(\vec{s})$; however, outside $e^{1}+e^{2}$, elements of $H(\vec{s})$ may intersect elements of $H(\vec{r})$ without including them. A word of warning: in the configuration of Fig. 7. the various sets $A_{i} \cdot e^{k}$ and $B_{j} \cdot e^{k}$ are all connected; however, the formula $\varphi_{\mathrm{W}}$ does not enforce this. That is, there is nothing to prevent the sets $e^{k}$ from chopping elements of $H(\vec{r})$ and $H(\vec{s})$ into several pieces.

Let $w^{*}$ be a fresh variable. The next conjuncts of $\varphi_{\mathrm{w}}$ ensure that the graphs $H(\vec{r})$ and $H(\vec{s})$ contain a common vertex, $w^{*}$ :

$$
\begin{array}{ll}
c\left(w^{*}\right) \wedge\left(w^{*} \neq 0\right), & \\
\operatorname{colour}\left(w^{*} ;\right. & \left.r_{0}, \ldots, r_{3}\right) \wedge \bigwedge_{i=0}^{3} \operatorname{colour}\left(r_{i} ; w^{*},-w^{*}\right), \\
\operatorname{colour}\left(w^{*} ;\right. & \left.s_{0}, \ldots, s_{3}\right) \wedge \bigwedge_{i=0}^{3} \operatorname{colour}\left(s_{i} ; w^{*},-w^{*}\right) .
\end{array}
$$




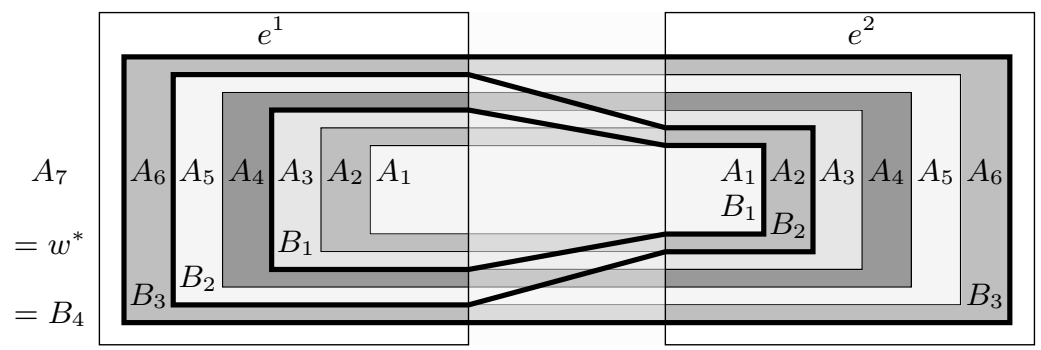

Figure 7: Arrangement satisfying $(10-15)$ such that $H(\vec{r})$ has nodes $A_{1}, \ldots, A_{7}$ (different shades) and $H(\vec{s})$ has nodes $B_{1}, \ldots, B_{4}$ (surrounded by thick lines); the unbounded region outside the largest of the depicted rectangles is $A_{7}=$ $B_{4}=w^{*}$.

To see why, note that the first conjunct of 14 ensures that $w^{*}$ is included in one of the sets $r_{i}$, and hence - since it is connected, by $(13)$ - in one of the vertices of $H(\vec{r})$; on the other hand, the remaining conjunct of (14) ensures that every vertex of $H(\vec{r})$ is included in either $w^{*}$ or $-w^{*}$. Since $w^{*}$ is non-empty, it must therefore be identical to a single vertex of $H(\vec{r})$. The same conclusion holds for $H(\vec{s})$ using $(15)$. In the arrangement of Fig. 77, we have $w^{*}=A_{7}=B_{4}$.

We need to impose a little more structure on the graphs $H(\vec{r})$ and $H(\vec{s})$. Let $r_{0}^{\prime}, \ldots, r_{3}^{\prime}$ and $w_{1}$ be fresh variables, and let $\varphi_{\mathrm{W}}$ contain the conjuncts:

$$
\begin{aligned}
& c\left(w_{1}\right) \wedge\left(w_{1} \leq r_{1}\right) \wedge\left(w_{1} \leq s_{1}\right) \wedge\left(w_{1} \cdot w^{*}=0\right), \\
& \bigwedge_{i=0}^{3}\left(r_{i}^{\prime} \leq r_{i}\right), \\
& \bigwedge_{k=1}^{2}\left(e^{k} \cdot r_{1}^{\prime} \cdot w_{1} \neq 0\right) .
\end{aligned}
$$

Since $w_{1}$ is a non-empty, connected subset of both $r_{1}$ and $s_{1}$, let $A_{1}$ be the component of $r_{1}$ including $w_{1}$, and let $B_{1}$ be the component of $s_{1}$ including $w_{1}$. It follows that $A_{1} \leq B_{1}$; the final conjunct of (16) ensures that $A_{1}$ and $B_{1}$ are both distinct from $w^{*}$. In the sequel, we shall construct a path in the graph $H(\vec{r})$ from $A_{1}$ to $w^{*}$, and a path in the graph $H(\vec{s})$ from $B_{1}$ to $w^{*}$. The proof will hinge on analysing the properties of these paths.

Let $t$ be a fresh variable, and let $\varphi_{\mathrm{W}}$ contain the conjuncts:

$$
\begin{aligned}
& \bigwedge_{k=1}^{2}\left(e^{k} \cdot t \neq 0\right) \\
& \bigwedge_{k=1}^{2} \bigwedge_{i=0}^{3} c\left(e^{k} \cdot\left(\left(r_{i}^{\prime} \cdot\left(-w^{*}\right)\right)+r_{\lfloor i+1\rfloor}+t\right)\right),
\end{aligned}
$$




$$
\begin{aligned}
& \bigwedge_{i=0}^{3} \neg C\left(r_{i}^{\prime}, t\right), \\
& \bigwedge_{i=0}^{3} \neg C\left(r_{i}^{\prime}, \quad r_{\lfloor i+1\rfloor} \cdot\left(-r_{\lfloor i+1\rfloor}^{\prime}\right)\right) .
\end{aligned}
$$

From (18), select a point $q_{1}^{k}$ in the interior of $e^{k} \cdot r_{1}^{\prime} \cdot w_{1}$. By (16), $q_{1}^{k} \notin w^{*}$. Let $X_{1}^{k}$ be the component of $e^{k} \cdot r_{1}$ containing $q_{1}^{k}$, and $A_{1}^{k}$ the component of $r_{1}$ including $X_{1}^{k}$; note that $A_{1}^{k}$ is a vertex of the graph $H(\vec{r})$. Evidently, $A_{1}^{k} \neq w^{*}$, and so $X_{1}^{k} \leq\left(e^{k} \cdot r_{1} \cdot\left(-w^{*}\right)\right)$. Now suppose $X_{i}^{k}$ has been defined and contains some point $q_{i}^{k} \in e^{k} \cdot r_{\lfloor i\rfloor}^{\prime} \cdot\left(-w^{*}\right)$. From (19]-21], $X_{i}^{k}$ contains a point $q_{i+1}^{k} \in e^{k} \cdot r_{\lfloor i+1\rfloor}$, which, by 22 , is in fact in $e^{k} \cdot r_{\lfloor i+1\rfloor}^{\prime}$. Let $X_{i+1}^{k}$ be the component of $e^{k} \cdot r_{\lfloor i+1\rfloor}$ containing $q_{i+1}^{k}$, and $A_{i+1}^{k}$ the component of $r_{\lfloor i+1\rfloor}$ including $X_{i+1}^{k}$; again, $A_{i+1}^{k}$ is a vertex of $H(\vec{r})$. Note that either $q_{i+1}^{k} \in w^{*}$ or $q_{i+1}^{k} \notin w^{*}$, and in the latter case, $q_{i+1}^{k} \in e^{k} \cdot r_{\lfloor i+1\rfloor}^{\prime} \cdot\left(-w^{*}\right)$. This process either continues forever, or, at some point, $q_{i+1}^{k} \in w^{*}$. But now consider any sequence $A_{1}^{k}, A_{2}^{k}, \ldots A_{\ell}^{k}$ obtained in this way. Evidently, $\left(A_{i}, A_{i+1}\right)$ is an edge of $H(\vec{r})$ for all $1 \leq i<\ell$; moreover, since $A_{i} \leq r_{\lfloor i\rfloor}$, we see from 10$]$ that $A_{i} \neq A_{i+2}$ for all $1 \leq i<\ell-1$, whence, since $H(\vec{r})$ is a tree, $A_{1}^{k}, A_{2}^{k}, \ldots A_{\ell}^{k}$ is a path (i.e., has no repeated nodes). It follows that, for some value of $i$, denoted by $n^{k}$, the condition $q_{i+1}^{k} \in w^{*}$ must hold, for otherwise, $H(\vec{r})$ would contain an infinite path, contradicting the assumption that the frame in question is finitely decomposible. Since $q_{n^{k}+1} \in r_{n^{k}+1}$, we have $A_{n^{k}+1}^{k}=w^{*}$, and hence, for $k=1,2$, there is a path $A_{1}^{k}, A_{2}^{k}, \ldots, A_{n^{k}+1}^{k}$ in $H(\vec{r})$ from $A_{1}^{k}=A_{1}$ to $A_{n^{k}+1}^{k}=w^{*}$. Indeed, this must be the same path for both $k=1,2$, so that we may drop the $k$-superscripts, and write:

$$
A_{1}, A_{2}, \ldots, A_{n}, A_{n+1}, \quad \text { where } A_{n+1}=w^{*} .
$$

(Note that the letter $n$ here is simply a convenient label for the length of this path: it has nothing to do with the dimension of the space.) It is important to remember that the sets $X_{i}^{1}$ and $X_{i}^{2}$, for a fixed value of $i$, will in general be distinct (Fig. 8).

Let us now turn our attention to the graph $H(\vec{s})$. Fix the value of $k$ for the moment $(1 \leq k \leq 2)$, and consider the sequence $X_{1}^{k}, \ldots, X_{n+1}^{k}$. Since $X_{i}^{k}$ is a connected subset of $e^{k} \cdot r_{\lfloor i\rfloor}$, it follows from (12) that each $X_{i}^{k}$ is included in some vertex of $H(\vec{s})$, say, $\hat{B}_{i}^{k}$. Thus, for $k=1$ and $k=2$, we have a sequence

$$
\hat{B}_{1}^{k}, \ldots, \hat{B}_{n+1}^{k}, \quad \text { where } A_{1} \leq \hat{B}_{1}^{k} \quad \text { and } \quad \hat{B}_{n+1}^{k}=w^{*} .
$$

Of course, these sequences may contain adjacent duplicates, since there is nothing to stop $X_{i}^{k}$ and $X_{i+1}^{k}$ being included in the same vertex of $H(\vec{s})$. Furthermore, the two sequences (for $k=1,2$ ) may be distinct, since, for fixed $i$, there is nothing to stop $X_{i}^{1}$ and $X_{i}^{2}$ lying in different vertices of $H(\vec{s})$; see Figs. 7 and 8 But now suppose we remove adjacent duplicates, obtaining sequences:

$$
B_{1}^{k}, \ldots, B_{m^{k}+1}^{k}, \quad \text { where } A_{1} \leq B_{1}^{k} \quad \text { and } \quad B_{m^{k}+1}^{k}=w^{*}
$$




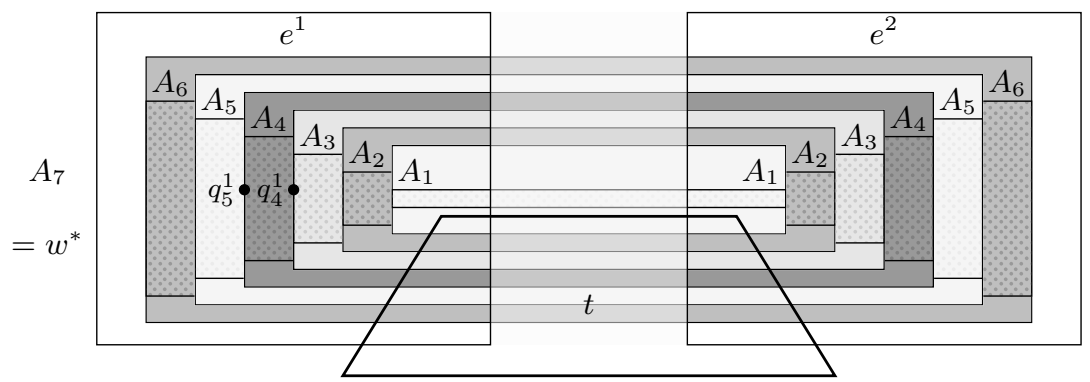

Figure 8: The regions $A_{1}, \ldots, A_{7}$ and $e^{1}, e^{2}$ from Fig. 7. Each region $A_{i}$ includes two components of $r_{\lfloor i\rfloor}^{\prime}$ : one lying in $e^{1}$, the other in $e^{2}$. Given a point $q_{i}^{k} \in$ $e^{k} \cdot r_{\lfloor i\rfloor}^{\prime} \cdot\left(-w^{*}\right)$, here illustrated for $i=4$ and $k=1$, formulas 17 -22 ensure the existence of a point $q_{i+1}^{k}$ in the same component of $e^{k} \cdot r_{\lfloor i\rfloor}$, and also in $e^{k} \cdot r_{\lfloor i+1\rfloor}^{\prime}$.

with $m^{k} \leq n$. Thus, every $B_{j}^{k}$ is the result of coalescing a contiguous block of identical vertices $\hat{B}_{i}^{k}$. Evidently, $\left(B_{j}^{k}, B_{j+1}^{k}\right)$ must be an edge of $H(\vec{s})$, for $1 \leq j \leq m^{k}$.

Now let $\varphi_{\mathrm{W}}$ contain the conjuncts:

$$
\bigwedge_{k=1}^{2} \bigwedge_{i=0}^{3} \bigwedge_{j=0}^{3} \neg C\left(e^{k} \cdot r_{i} \cdot s_{j}, \quad e^{k} \cdot r_{\lfloor i+1\rfloor} \cdot s_{\lfloor j-1\rfloor}\right) .
$$

We claim that, in that case, $B_{j}^{k} \leq s_{\lfloor j\rfloor}$ for all $1 \leq j \leq m^{k}$. The proof is by induction on $j$. By (16), $X_{1}^{k} \leq s_{1}$, and therefore $B_{1}^{k} \leq s_{1}$. Suppose, then, $B_{j}^{k} \leq s_{\lfloor j\rfloor}$ for some $1 \leq j<m^{k}$. Let $\hat{B}_{i}^{k}$, be the last vertex in the block coalescing to $B_{j}^{k}$, so that $\hat{B}_{i+1}^{k}$ is the first element of the block coalescing to $B_{j+1}^{k}$. Thus, $X_{i}^{k} \leq r_{\lfloor i\rfloor}$ and $X_{i+1}^{k} \leq r_{\lfloor i+1\rfloor}$. But (11) and (23) then ensure that either $B_{j+1}^{k} \leq s_{\lfloor j\rfloor}$ or $B_{j+1}^{k} \leq s_{\lfloor j+1\rfloor}$; and the former is impossible, since then $\hat{B}_{i}^{k}$ and $\hat{B}_{i+1}^{k}$ would have coalesced to the same block. This proves the claim. By (11) (and the fact that $B_{m^{k}-1}^{k} \neq w^{*}$ ), we then have $B_{j}^{k} \neq B_{j+2}^{k}$ for all $1 \leq j<m^{k}$. And since $H(\vec{s})$ is a tree, it follows that $B_{1}^{k}, \ldots, B_{m^{k}+1}^{k}$ is a path through $H(\vec{s})$ with $B_{1}^{1}=B_{1}^{2}$ and $B_{m^{1}+1}^{1}=B_{m^{2}+1}^{2}=w^{*}$. Indeed, this is the same path through $H(\vec{s})$ for both values of $k$, so that we can again drop the superscripts, and just write

$$
B_{1}, \ldots, B_{m}, B_{m+1}, \quad \text { where } B_{m+1}=w^{*} .
$$

Taking stock, we see that, for each $k=1,2$, the path $A_{1}, \ldots, A_{n}$ may be grouped into $m$ contiguous blocks $E_{1}^{k}, \ldots, E_{m}^{k}$ by taking the vertex $A_{i}$ to be in the $j$ th block $E_{j}^{k}$ just in case $e^{k} \cdot X_{i}^{k} \leq B_{j}$. We may depict this grouping as 
follows:

$$
A_{1}, \ldots, A_{n}=\underbrace{A_{1,1}^{k}, \ldots, A_{1, h_{1}^{k}}^{k}}_{E_{1}^{k}}, \ldots, \underbrace{A_{j, 1}^{k}, \ldots, A_{j, h_{j}^{k}}^{k}}_{E_{j}^{k}}, \ldots, \underbrace{A_{m, 1}^{k}, \ldots, A_{m, h_{m}^{k}}^{k}}_{E_{m}^{k}} .
$$

It is important to realize that, although there is only one path $A_{1}, \ldots, A_{n+1}$ and one path $B_{1}, \ldots, B_{m+1}$, the two values $k=1$ and $k=2$ will in general give rise to different groupings of the vertices of the former into blocks corresponding to the vertices of the latter (hence, the two sequences of indices $h_{1}^{k}, \ldots, h_{m}^{k}$ ).

Recall the PCP-instance $\mathrm{W}=\left(\mathrm{w}^{1}, \mathrm{w}^{2}\right)$ over the alphabets $T$ and $U$, which we wish to encode. We regard the elements of these alphabets as fresh variables, and order them in some way to form tuples $\vec{t}$ and $\vec{u}$. We use these variables to colour the vertices of $H(\vec{s})$ and $H(\vec{r})$, respectively, by taking $\varphi_{\mathrm{W}}$ to contain the conjuncts:

$$
\begin{aligned}
& \operatorname{part}(\vec{t}) \wedge \bigwedge_{j=0}^{3} \operatorname{colour}\left(s_{j} ; \vec{t}\right), \\
& \operatorname{part}(\vec{u}) \wedge \bigwedge_{i=0}^{3} \operatorname{colour}\left(r_{i} ; \vec{u}\right) .
\end{aligned}
$$

In this way, the path $A_{1}, \ldots, A_{n}$ defines a word $v \in U^{*}$, and the path $B_{1}, \ldots, B_{m}$ defines a word $\tau \in T^{*}$. Using the groupings of the sequence $A_{1}, \ldots, A_{n}$ obtained above, we shall write conjuncts of $\varphi_{\mathrm{w}}$ ensuring that $\mathrm{w}^{k}(\tau)=v$ for $k=1,2$. This will mean that, if $\varphi_{\mathrm{W}}$ has a satisfying assignment over some frame $\mathcal{S} \in \mathcal{K}$, then the $\mathrm{PCP}$-instance $\mathrm{W}$ is positive.

For $k=1,2$, let $\left\{p_{h, \ell}^{k}|1 \leq h \leq| T|, 1 \leq \ell \leq| \mathrm{w}^{k}\left(t_{h}\right) \mid\right\}$, be a collection of fresh variables, enumerated in some way as $\vec{p}^{k}$, which we shall use to colour the vertices of $H(\vec{r})$. That is, we add to $\varphi_{\mathrm{W}}$ the conjuncts:

$$
\bigwedge_{k=1}^{2}\left(\operatorname{part}\left(\vec{p}^{k}\right) \wedge \bigwedge_{i=0}^{3} \operatorname{colour}\left(r_{i} ; \vec{p}^{k}\right)\right)
$$

We refer to these variables as position colours, because we are to think of $p_{h, \ell}^{k}$ as denoting the $\ell$ th position in the word $\mathrm{w}^{k}\left(t_{h}\right)$. In particular, any position colour $p_{h, \ell}^{k}$ is naturally associated to the letter $t_{h}$ of $T$. Fixing $k$ for the moment, consider the vertices $A_{j, 1}^{k}, \ldots, A_{j, h_{j}^{k}}^{k}$ grouped into the $j$ th block, $E_{j}^{k}$. What ensures that these vertices belong to one block is the existence of a single $B_{j}$ such that, if $A_{i}$ is one of these vertices, then the corresponding set $X_{i}^{k} \leq A_{i}$ is included in $B_{j}$. By (24), $X_{i}^{k} \leq t_{h}$, for some $1 \leq h \leq|T|$. It follows that the conjuncts

$$
\bigwedge_{k=1}^{2} \bigwedge_{h=1}^{|T|}\left(t_{h} \leq \sum_{\ell=1}^{\left|w^{k}\left(t_{h}\right)\right|} p_{h, \ell}^{k}\right)
$$


ensure that the vertices in this block are assigned 'position' colours associated to the common tile $t_{h}$.

We proceed to write constraints ensuring that these colours are assigned in exactly the canonical order: $p_{h, 1}^{k}, \ldots p_{h,\left|w^{k}\left(t_{h}\right)\right|}^{k}$ (from which, incidentally, it follows that $\left|\mathrm{w}^{k}\left(t_{h}\right)\right|=h_{j}^{k}$ ). We begin by adding to $\varphi \mathrm{w}$ the conjuncts:

$$
\bigwedge_{k=1}^{2} \bigwedge_{h=1}^{|T|} \bigwedge_{\ell=2}^{\left|\mathrm{w}^{k}\left(t_{h}\right)\right|}\left(\left(w_{1} \cdot p_{h, \ell}^{k}=0\right) \wedge \bigwedge_{i=0}^{3} \neg C\left(s_{i}, s_{\lfloor i+1\rfloor} \cdot p_{h, \ell}^{k}\right)\right) .
$$

These ensure that the first vertex of each block is assigned one of the colours $p_{h, 1}^{k}$, for $1 \leq h \leq|T|$. The rules for colouring successive vertices can now be simply stated. Consider the following binary relation on the variables in $\vec{p}^{k}$ :

$$
\begin{aligned}
\Psi^{k}=\left\{\left(p_{h, \ell}^{k}, p_{h, \ell+1}^{k}\right)|1 \leq h \leq| T \mid, 1\right. & \left.\leq \ell<\left|\mathrm{w}^{k}\left(t_{h}\right)\right|\right\} \\
& \cup\left\{\left(p_{h,\left|\mathrm{w}^{k}\left(t_{h}\right)\right|}^{k}, p_{h^{\prime}, 1}^{k}\right)\left|1 \leq h, h^{\prime} \leq\right| T \mid\right\} .
\end{aligned}
$$

This relation captures the rules of possible succession for colouring by the variables $p_{h, \ell}^{k}:(a)$ if $A_{i}$ is coloured $p_{h, \ell}^{k}$, where $\ell$ indicates a non-final position in the word $\mathrm{w}^{k}\left(t_{h}\right)$, then $A_{i+1}$ must be coloured $p_{h, \ell+1}^{k} ;(b)$ if $A_{i}$ is coloured $p_{h,\left|\mathrm{w}^{k}\left(t_{h}\right)\right|}^{k}$, indicating the final position in the word $\mathrm{w}^{k}\left(t_{h}\right)$, then $A_{i+1}$ must be coloured $p_{h^{\prime}, 1}^{k}$ for some $1 \leq h^{\prime} \leq|T|$. We therefore add to $\varphi_{\mathrm{W}}$ the conjuncts

$$
\bigwedge_{k=1}^{2} \bigwedge_{i=0}^{3} \bigwedge_{\left(p_{h, \ell}^{k}, p_{h^{\prime}, \ell^{\prime}}^{k}\right) \notin \Psi^{k}} \neg C\left(p_{h, \ell}^{k} \cdot r_{i}, \quad p_{h^{\prime}, \ell^{\prime}}^{k} \cdot r_{\lfloor i+1\rfloor}\right) .
$$

We also ensure that each block spells out only one word. That is, we ensure that no vertex of the sequence $A_{1}, \ldots, A_{n}$ can be coloured with the starting position in a word if the previous vertex belongs to the same block:

$$
\bigwedge_{k=1}^{2} \bigwedge_{i=0}^{3} \bigwedge_{j=0}^{3} \bigwedge_{h=1}^{|T|} \neg C\left(r_{i} \cdot s_{j}, \quad r_{\lfloor i+1\rfloor} \cdot s_{j} \cdot p_{h, 1}^{k}\right) .
$$

Lastly, we ensure that the final vertex of the final block corresponds to the final position in a word. In other words, we ensure that the vertex $A_{n}$ (which contacts $\left.A_{n+1}=w^{*}\right)$ is coloured $p_{h,\left|w^{k}\left(t_{h}\right)\right|}^{k}$, for some $1 \leq h \leq|T|$ :

$$
\bigwedge_{k=1}^{2} \bigwedge_{h=1}^{|T|} \bigwedge_{\ell=1}^{\left(\left|w^{k}\left(t_{h}\right)\right|-1\right)} \neg C\left(p_{h, \ell}^{k}, w^{*}\right) .
$$

At this stage, we have ensured that, for $k=1$ and $k=2$, vertices of each block $A_{j, 1}^{k}, \ldots, A_{j, h_{j}^{k}}^{k}, 1 \leq j \leq m$, are coloured $p_{h, 1}^{k}, \ldots, p_{h,\left|\mathrm{w}^{k}\left(t_{h}\right)\right|}^{k}$, where $t_{h}$ is the $j$ th letter of the word $\tau$. This easily enables us to enforce the sought-after conditions $\mathrm{w}^{k}(\tau)=v$ for $k=1,2$. Denoting by $u_{h, \ell}^{k}$ the variable in $\vec{u}$ (i.e. that 
letter of the alphabet $U$ ) that is the $\ell$ th letter in the word $\mathrm{w}^{k}\left(t_{h}\right)$, we add to $\varphi_{\mathrm{W}}$ the conjuncts:

$$
\bigwedge_{k=1}^{2} \bigwedge_{h=1}^{|T|} \bigwedge_{\ell=1}^{\left|w^{k}\left(t_{h}\right)\right|}\left(p_{h, \ell}^{k} \leq u_{h, \ell}^{k}\right) .
$$

That $\mathrm{w}^{k}(\tau)=v$ for $k=1,2$ then follows from the fact that each vertex $A_{i}$ is assigned a unique colour from $\vec{u}$. Thus, if $\varphi_{\mathrm{W}}$ is satisfiable over $\mathcal{K}$ then $\mathrm{W}$ is positive.

Conversely, if $\mathrm{W}$ is positive, it is obvious that $\varphi_{\mathrm{W}}$ may be satisfied over $\operatorname{RCP}\left(\mathbb{R}^{n}\right), n \geq 2$, by suitably extending a configuration similar to that shown in Fig. 7

Corollary 9. Let $\mathcal{K}$ be any class of unicoherent, finitely decomposible frames, such that $\mathcal{K}$ contains some frame of the form $\left(\mathbb{R}^{n}, \mathcal{S}\right), n \geq 2$, where $\operatorname{RCP}\left(\mathbb{R}^{n}\right) \subseteq$ $\mathcal{S}$. Then the problem $\operatorname{Sat}\left(\mathcal{C} c^{\circ}, \mathcal{K}\right)$ is r.e.-hard.

Proof. We start with the formula $\varphi_{\mathrm{W}}$ of Theorem 8 , and replace all occurrences of $c$ by $c^{\circ}$. Denote the resulting $\mathcal{C} c^{\circ}$-formula by $\varphi_{\mathrm{W}}^{\circ}$. Since all atoms of the form $c(\tau)$ in $\varphi_{\mathrm{W}}$ occur with positive polarity, $\varphi_{\mathrm{W}}^{\circ}$ entails $\varphi_{\mathrm{W}}$. On the other hand, by inspection of Fig. 7, we see that if $\mathrm{W}$ is positive, then $\varphi_{\mathrm{W}}^{\circ}$ will be satisfiable in $\operatorname{RCP}\left(\mathbb{R}^{2}\right)$, and hence in $\operatorname{RCP}\left(\mathbb{R}^{n}\right)$ for all $n \geq 2$. This proves the corollary.

Corollary 10. Let $\mathcal{K}$ be any class of unicoherent, finitely decomposible frames, such that $\mathcal{K}$ contains some frame of the form $\left(\mathbb{R}^{n}, \mathcal{S}\right), n \geq 2$, where $\operatorname{RCP}\left(\mathbb{R}^{n}\right) \subseteq$ $\mathcal{S}$. Then the problem $\operatorname{Sat}(\mathcal{B} c, \mathcal{K})$ is r.e.-hard.

Proof. Consider again the formula $\varphi_{\mathrm{w}}$ of Theorem 8. Since all occurrences of the predicate $C$ in $\varphi_{\mathrm{W}}$ have negative polarity, we can replace them with $\mathcal{B}_{c}$ formulas as we did in the proof of Theorem 7. The resulting formula $\varphi_{\mathrm{W}}^{c}$ implies $\varphi_{\mathrm{W}}$, and is satisfiable in $\operatorname{RCP}\left(\mathbb{R}^{n}\right)$, for all $n \geq 2$, whenever $\mathrm{W}$ is positive.

\section{Undecidability: the plane case}

In Sec. 3. we established that, if $\mathcal{L}$ is any of the languages $\mathcal{B} c, \mathcal{C} c$ or $\mathcal{C} c^{\circ}$, there exists an $\mathcal{L}$-formula that is satisfiable over $\mathrm{RC}\left(\mathbb{R}^{n}\right), n \geq 2$, but only by regions having infinitely many components. Nothing was mentioned in this regard about the language $\mathcal{B} c^{\circ}$. In Sec. 4. we established the undecidability of $\operatorname{Sat}\left(\mathcal{L}, \operatorname{RCP}\left(\mathbb{R}^{n}\right)\right), n \geq 2$, where $\mathcal{L}$ is any of the languages $\mathcal{B} c, \mathcal{C} c, \mathcal{C} c^{\circ}$. Nothing was mentioned in this regard about the problems $\operatorname{Sat}\left(\mathcal{B} c^{\circ}, \operatorname{RCP}\left(\mathbb{R}^{n}\right)\right), n \geq 2$, or indeed about the problems $\operatorname{Sat}\left(\mathcal{L}, \operatorname{RC}\left(\mathbb{R}^{n}\right)\right)$ where $\mathcal{L}$ is any of $\mathcal{B} c, \mathcal{B} c^{\circ}, \mathcal{C} c$, $\mathcal{C} c^{\circ}$. In this section, we complete the picture in the case $n=2$. Specifically, we establish the existence of a $\mathcal{B} c^{\circ}$-formula satisfiable over $\mathrm{RC}\left(\mathbb{R}^{2}\right)$, but only by regions having infinitely many components; and we establish the undecidability of the problems $\operatorname{Sat}\left(\mathcal{L}, \operatorname{RC}\left(\mathbb{R}^{2}\right)\right)$ and $\operatorname{Sat}\left(\mathcal{L}, \operatorname{RCP}\left(\mathbb{R}^{2}\right)\right)$, where $\mathcal{L}$ is any of $\mathcal{B} c, \mathcal{B} c^{\circ}$, $\mathcal{C} c$ or $\mathcal{C} c^{\circ}$. 
We employ the standard terminology of Jordan arcs and curves: a nondegenerate Jordan arc is a continuous, 1-1 function $\alpha$ from the unit interval to $\mathbb{R}^{2}$; a degenerate Jordan arc is a constant function from the unit interval to $\mathbb{R}^{2}$; a Jordan arc is a degenerate Jordan arc or a non-degenerate Jordan arc. A Jordan curve is a continuous, 1-1 function from the unit circle to $\mathbb{R}^{2}$. Where no confusion results, we identify Jordan arcs and curves with their loci (ranges). If $\alpha_{1}$ and $\alpha_{2}$ are Jordan arcs which intersect in the unique point $\alpha_{1}(1)=\alpha_{2}(0)$, then we write $\alpha_{1} \alpha_{2}$ to denote, ambiguously, any Jordan $\operatorname{arc} \alpha$ with locus $\alpha_{1} \cup \alpha_{2}$ such that $\alpha(0)=\alpha_{1}(0)$ and $\alpha(1)=\alpha_{2}(1)$. We employ the following notation: if $\alpha$ is a Jordan arc, $\alpha^{-1}$ denotes a Jordan arc with the same locus but opposite direction, e.g., $\alpha^{-1}(t)=\alpha(1-t)$, for all $0 \leq t \leq 1$. If, in addition, $p_{1}=\alpha\left(t_{1}\right)$, $p_{2}=\alpha\left(t_{2}\right)$ are points on $\alpha$ with $t_{2} \geq t_{1}, \alpha\left[p_{1}, p_{2}\right]$ denotes a Jordan arc whose locus is the segment of $\alpha$ between $p_{1}$ and $p_{2}$, and which has the same direction as $\alpha: \alpha\left[p_{1}, p_{2}\right](t)=\alpha\left(t_{1}+t\left(t_{2}-t_{1}\right)\right)$. An end-cut to $p$ in a set $X$ is a Jordan $\operatorname{arc} \alpha \subseteq X^{\circ} \cup\{p\}$ such that $\alpha(1)=p$. A cross-cut in $X$ is a Jordan $\operatorname{arc} \alpha$ in $X$ intersecting the boundary $\delta X$ of $X$ only at its endpoints $\alpha(0)$ and $\alpha(1)$.

\subsection{Forcing infinitely many components with $\mathcal{B} c^{\circ}$}

We begin by showing that there exists a $\mathcal{B} c^{\circ}$-formula that is satisfiable over $\mathrm{RC}\left(\mathbb{R}^{2}\right)$, but only by regions having infinitely many components. Many of the techniques we employ will prove useful in Sec. 5.2. Our basic tools are two formulas that enable us to construct Jordan arcs and curves containing points in specified regions. But before presenting these formulas, we need to establish the following property of regular closed sets:

Lemma 11. Let $T$ be any topological space, and $a, b_{1}$ and $b_{2}$ elements of $\mathrm{RC}(T)$ such that $b_{1} \cdot b_{2}=0$. Then $\left(a+b_{1}\right)^{\circ} \cap\left(a+b_{2}\right)^{\circ}=a^{\circ}$.

Proof. Note that, for any $s \in \mathrm{RC}(T),-s=T \backslash s^{\circ}$. Since $b_{1} \cdot b_{2}=0$, we have $-a=\sum_{i=1,2}\left(-\left(a+b_{i}\right)\right)$, which is then equal to $\bigcup_{i=1,2}\left(T \backslash\left(a+b_{i}\right)^{\circ}\right)=$ $T \backslash\left(\bigcap_{i=1,2}\left(a+b_{i}\right)^{\circ}\right)$.

Consider now the following $\mathcal{B} c^{\circ}$-formula:

$$
\operatorname{frame}^{\circ}\left(r_{0}, \ldots, r_{n-1}\right)=\bigwedge_{i=0}^{n-1}\left(\left(r_{i} \neq 0\right) \wedge c^{\circ}\left(r_{i}+r_{\lfloor i+1\rfloor}\right)\right) \wedge \bigwedge_{0 \leq i<j<n}\left(r_{i} \cdot r_{j}=0\right),
$$

where $\lfloor k\rfloor$ denotes the value of $k$ modulo $n$. This formula allows us to construct Jordan curves that contain points of all regions $r_{0}, \ldots, r_{n-1}$ :

Lemma 12. Fix $n \geq 3$, and let $\left(a_{0}, \ldots, a_{n-1}\right)$ be a tuple of elements of $\mathrm{RC}\left(\mathbb{R}^{2}\right)$ satisfying frame ${ }^{\circ}\left(r_{0}, \ldots, r_{n-1}\right)$. Then there exist Jordan arcs $\alpha_{0}, \ldots, \alpha_{n-1}$ and points $p_{0}, \ldots, p_{n-1}$ such that: for all $0 \leq i<n, \alpha_{i}$ is a Jordan arc from $p_{i}$ to $p_{\lfloor i+1\rfloor}$, with $\alpha_{i} \subseteq\left(a_{i}+a_{\lfloor i+1\rfloor}\right)^{\circ} ; \alpha_{0} \cdots \alpha_{n-1}$ is a Jordan curve lying in $\left(a_{0}+\cdots+a_{n-1}\right)^{\circ}$; and $p_{i} \in a_{i}^{\circ}$, for all $0 \leq i<n$. 
Proof. For every $0 \leq i<n$, select points $p_{i}^{\prime}$ in the interior of $a_{i}$ and connect each $p_{i}^{\prime}$ to $p_{\lfloor i+1\rfloor}^{\prime}$ with an $\operatorname{arc} \alpha_{i}^{\prime \prime} \subseteq\left(a_{i}+a_{\lfloor i+1\rfloor}\right)^{\circ}$. Let $p_{1}$ be the first point on $\alpha_{0}^{\prime \prime}$ that is on $\alpha_{1}^{\prime \prime}$, let $\alpha_{0}^{\prime}$ be the initial segment of $\alpha_{0}^{\prime \prime}$ ending at $p_{1}$, and let $\alpha_{1}^{\prime}$ be the final segment of $\alpha_{1}^{\prime \prime}$ starting at $p_{1}$. Note that $\alpha_{0}^{\prime} \cap \alpha_{1}^{\prime}=\left\{p_{1}\right\}$. For $2 \leq i<n-1$, let $p_{i}$ be the first point on $\alpha_{i-1}^{\prime}$ that is on $\alpha_{i}^{\prime \prime}$, let $\alpha_{i-1}$ be the initial segment of $\alpha_{i-1}^{\prime}$ ending at $p_{i}$, and let $\alpha_{i}^{\prime}$ be the final segment of $\alpha_{i}^{\prime \prime}$ starting at $p_{i}$. Note that $\alpha_{i-1} \cap \alpha_{i}^{\prime}=\left\{p_{i}\right\}$. Finally, let $p_{0}$ be the first point on $\alpha_{n-1}^{\prime}$ that is on $\alpha_{0}^{\prime}$, let $\alpha_{n-1}$ be the initial segment of $\alpha_{n-1}^{\prime}$ ending at $p_{0}$, and let $\alpha_{0}$ be the final segment of $\alpha_{0}^{\prime}$ starting at $p_{0}$. Note that $\alpha_{n-1} \cap \alpha_{0}=\left\{p_{0}\right\}$. By construction, for every $0 \leq i<n, \alpha_{i}$ connects points $p_{i}$ and $p_{\lfloor i+1\rfloor}$, and $\alpha_{\lfloor i-1\rfloor} \cap \alpha_{i}=\left\{p_{i}\right\}$, whence, by Lemma 11, $p_{i} \in a_{i}^{\circ}$.

Consider now the $\mathcal{C} c^{\circ}$-formula, for $n>1$,

$$
\begin{aligned}
& \operatorname{stack}^{\circ}\left(r_{1}, \ldots, r_{n}\right)= \\
& \bigwedge_{i=1}^{n} c^{\circ}\left(r_{i}+\cdots+r_{n}\right) \wedge \bigwedge_{1 \leq i<j \leq n}\left(r_{i} \cdot r_{j}=0\right) \wedge \bigwedge_{\substack{1 \leq i<j \leq n \\
j-i>1}} \neg C\left(r_{i}, r_{j}\right),
\end{aligned}
$$

which will allow us to construct arcs containing points of all the regions $r_{1}, \ldots, r_{n}$ :

Lemma 13. Let $\left(a_{1}, \ldots, a_{n}\right)$ be a tuple of elements of $\mathrm{RC}\left(\mathbb{R}^{2}\right)$ satisfying $\operatorname{stack}^{\circ}\left(r_{1}, \ldots, r_{n}\right)$. Then every point $p_{1} \in a_{1}^{\circ}$ can be connected to every point $p_{n} \in a_{n}^{\circ}$ by a Jordan arc $\alpha=\alpha_{1} \cdots \alpha_{n-1}$ such that, for all $1 \leq i<n, \alpha_{i}$ is a non-degenerate Jordan arc in $\left(a_{i}+a_{i+1}\right)^{\circ}$, starting at a point $p_{i} \in a_{i}^{\circ}$.

Proof. Since $a_{1}+\cdots+a_{n}$ is interior-connected, let $\alpha_{1}^{\prime} \subseteq\left(a_{1}+\cdots+a_{n}\right)^{\circ}$ be a Jordan arc connecting $p_{1}$ to $p_{n}$. Since $\neg C\left(a_{1},\left(a_{3}+\cdots+a_{n}\right)\right)$, $\alpha_{1}^{\prime}$ must contain a point $p_{1}^{\prime} \in a_{2}^{\circ}$ such that $\alpha_{1}^{\prime}\left[p_{1}, p_{1}^{\prime}\right] \subseteq\left(a_{1}+a_{2}\right)^{\circ}$. For convenience, let $p_{0}=p_{1}$, let $\alpha_{0}$ be the degenerate Jordan arc located at $p_{1}$, and let $a_{0}$ be the empty region.

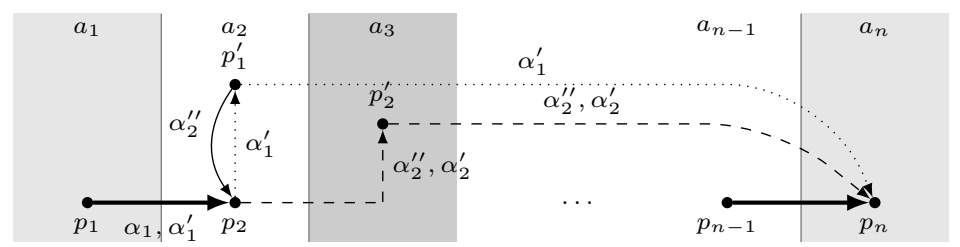

Figure 9: The constraint $\operatorname{stack}^{\circ}\left(a_{1}, \ldots, a_{n}\right)$ ensures the existence of a Jordan arc $\alpha=\alpha_{1} \cdots \alpha_{n-1}$ which connects a point $p_{1} \in a_{1}^{\circ}$ to a point $p_{n} \in a_{n}^{\circ}$.

We inductively define, for all $1 \leq i<n$, $\operatorname{arcs} \alpha_{i-1}, \alpha_{i}^{\prime}$ and points $p_{i}, p_{i}^{\prime}$ with the following properties: $\alpha_{i-1} \subseteq\left(a_{i-1}+a_{i}\right)^{\circ}$ and runs from $p_{i-1}$ to $p_{i} ; \alpha_{i}^{\prime} \subseteq$ $\left(a_{i}+\cdots+a_{n}\right)^{\circ} ; \alpha_{0} \cdots \alpha_{i-1} \alpha_{i}^{\prime}$ is a Jordan arc from $p_{0}$ to $p_{n} ;$ and $p_{i}^{\prime} \in \alpha_{i}^{\prime} \cap a_{i+1}^{\circ}$ with $\alpha_{i}^{\prime}\left[p_{i}, p_{i}^{\prime}\right] \subseteq\left(a_{i}+a_{i+1}\right)^{\circ}$. Suppose that, for some $1 \leq i \leq n-2$, the requisite entities have already been defined (notice that this is already the case for $i=1$ ). 
Since $a_{i+1}+\cdots+a_{n}$ is interior-connected, let $\alpha_{i+1}^{\prime \prime} \subseteq\left(a_{i+1}+\cdots+a_{n}\right)^{\circ}$ be a Jordan arc connecting $p_{i}^{\prime}$ to $p_{n}$. Since we certainly have $\left(a_{1}+\cdots+a_{i}\right) \cdot\left(a_{i+1}+\right.$ $\left.\cdots+a_{n}\right)=0, \alpha_{i+1}^{\prime \prime}$ can intersect $\alpha_{0} \cdots \alpha_{i-1} \alpha_{i}^{\prime}$ only in its final segment $\alpha_{i}^{\prime}$. Let $p_{i+1}$ be the first point of $\alpha_{i}^{\prime}$ lying on $\alpha_{i+1}^{\prime \prime}$; let $\alpha_{i}$ be the initial segment of $\alpha_{i}^{\prime}$ ending at $p_{i+1}$; and let $\alpha_{i+1}^{\prime}$ be the final segment of $\alpha_{i+1}^{\prime \prime}$ starting at $p_{i+1}$. By construction, then, $\alpha_{0} \cdots \alpha_{i} \alpha_{i+1}^{\prime}$ is a Jordan arc from $p_{0}$ to $p_{n}$, and $\alpha_{i} \subseteq\left(a_{i}+a_{i+1}\right)^{\circ}$. Moreover, since $\neg C\left(a_{i+1},\left(a_{i+3}+\cdots+a_{n}\right)\right), \alpha_{i+1}^{\prime}$ must contain a point $p_{i+1}^{\prime} \in a_{i+2}^{\circ}$ such that $\alpha_{i+1}^{\prime}\left[p_{i+1}, p_{i+1}^{\prime}\right] \subseteq\left(a_{i+1}+a_{i+2}\right)^{\circ}$. Continuing up to the value $i=n-1$, we have defined $\alpha_{1}, \ldots, \alpha_{n-2}, \alpha_{n-1}^{\prime}$ and $p_{1}, \ldots, p_{n-1}$. It remains only to define $\alpha_{n-1}$; for this we simply set $\alpha_{n-1}=\alpha_{n-1}^{\prime}$.

For all $1<i<n$, we have $p_{i} \in \alpha_{i-1} \cap \alpha_{i}$, whence, by Lemma $11, p_{i} \in a_{i}^{\circ}$. It also follows that the $\alpha_{i}$ are non-degenerate.

It should be noted that $\operatorname{stack}^{\circ}\left(r_{1}, \ldots, r_{n}\right)$ is a not a $\mathcal{B} c^{\circ}$-formula, as it contains (negative) occurrences of the contact predicate $C$. It turns out, however, that we can eliminate them. To this end, consider the $\mathcal{B} c^{\circ}$-formula

$$
\begin{array}{r}
\mathrm{K} 5 \mathrm{~m}\left(r_{1}, \ldots, r_{5}\right)=\bigwedge_{i=1}^{5}\left(c^{\circ}\left(r_{i}\right) \wedge\left(r_{i} \neq 0\right)\right) \wedge \bigwedge_{1 \leq i<j \leq 5}\left(r_{i} \cdot r_{j}=0\right) \wedge \\
\bigwedge_{j=3}^{5} c^{\circ}\left(r_{1}+r_{j}\right) \wedge \bigwedge_{2 \leq i<j \leq 5} c^{\circ}\left(r_{i}+r_{j}\right) .
\end{array}
$$

This formula is similar to formula (2) encoding the non-planar graph $K_{5}$ (hence the name); however, there is no requirement that $r_{1}+r_{2}$ is interior-connected.

Lemma 14. ( $i)$ For each tuple $\left(a_{1}, \ldots, a_{5}\right)$ of elements of $\mathrm{RC}\left(\mathbb{R}^{2}\right)$ satisfying $\operatorname{K5m}\left(r_{1}, \ldots, r_{5}\right)$, we have $\neg C\left(a_{1}, a_{2}\right)$. (ii) If regions $b_{1}$ and $b_{2}$ can be separated by a Jordan curve then there exist polygons $\left(a_{1}, \ldots, a_{5}\right)$ satisfying $\mathrm{K} 5 \mathrm{~m}\left(r_{1}, \ldots, r_{5}\right)$ such that $b_{1} \leq a_{1}$ and $b_{2} \leq a_{2}$.

Proof. ( $i)$ For all $i(1 \leq i \leq 5)$, pick a point $p_{i} \in a_{i}^{\circ}$. Then, for all $j(3 \leq j \leq 5)$ let $\gamma_{1, j}$ be an arc from $p_{1}$ to $p_{j}$ lying in $\left(a_{1}+a_{j}\right)^{\circ}$, and, for all $i, j(2 \leq i<j \leq 5)$, let $\gamma_{i, j}$ be an arc from $p_{i}$ to $p_{j}$ lying in $\left(a_{i}+a_{j}\right)^{\circ}$. It is routine to show that the various $\gamma_{i, j}$ can be chosen so that they intersect only at their endpoints. Thus, $\Gamma=\gamma_{3,4} \gamma_{4,5} \gamma_{3,5}^{-1}$ forms a Jordan curve in $\left(a_{3}+a_{4}+a_{5}\right)^{\circ}$, and the arcs $\gamma_{2,3}, \gamma_{2,4}$ and $\gamma_{2,5}$ join $\Gamma$ to the point $p_{2}$ lying in one of its residual domains. (Fig. 10 illustrates the situation where $p_{2}$ lies in the bounded residual domain.) Since $a_{1}$ and $a_{2}$ are (interior-) connected and cannot intersect $\Gamma$, they each lie in one of its residual domains. It suffices to show that $a_{1}$ and $a_{2}$ lie in different residual domains. To see this, observe that the arcs $\gamma_{2,3}, \gamma_{2,4}$ and $\gamma_{2,5}$ divide the residual domain of $\Gamma$ containing $p_{2}$ into three regions, bounded by arcs lying in $\left(a_{2}+a_{3}+a_{4}\right)^{\circ},\left(a_{2}+a_{4}+a_{5}\right)^{\circ}$ and $\left(a_{2}+a_{5}+a_{3}\right)^{\circ}$, respectively. But if $a_{1}$ and $a_{2}$ lie on the same side of $\Gamma$, then $p_{1}$ lies in one of these regions, contradicting the existence of arcs $\gamma_{1, j} \subseteq\left(a_{1}+a_{j}\right)^{\circ}$ connecting $p_{1}$ to $p_{j}$; for $j=3,4,5$.

(ii) Let $\Gamma$ be a Jordan curve separating $b_{1}$ and $b_{2}$. We may assume that $\Gamma$ is piecewise-linear. Now thicken $\Gamma$ to form an annular element of $\operatorname{RCP}\left(\mathbb{R}^{2}\right)$, 


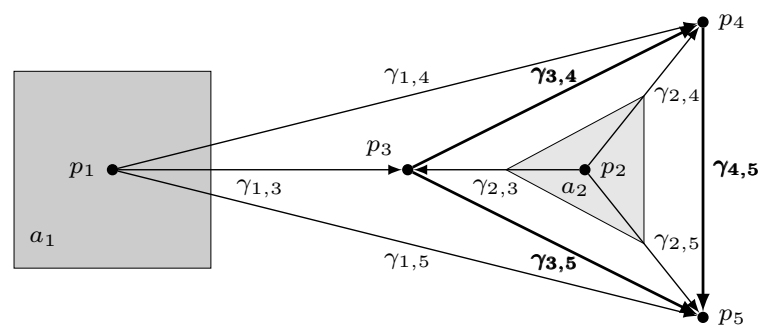

Figure 10: The Jordan curve $\Gamma=\gamma_{3,4} \gamma_{4,5} \gamma_{3,5}^{-1}$ (thick lines) separating $a_{1}$ from $a_{2}$.

still disjoint from $b_{1}$ and $b_{2}$, and divide it into the three interior-connected and non-overlapping polygons $a_{3}, a_{4}, a_{5}$. Choose $a_{1}$ and $a_{2}$ to be the components of the complement of $a_{3}+a_{4}+a_{5}$ containing $b_{1}$ and $b_{2}$, respectively.

We remark that Lemma 14 guarantees that $\left(a_{1}, \ldots, a_{5}\right)$ are regular closed polygons. This fact will be important in Sec. 5.2, where we prove the undecidability of $\operatorname{Sat}\left(\mathcal{B} c^{\circ}, \operatorname{RCP}\left(\mathbb{R}^{2}\right)\right)$; for the main result of this section, however, we require only that $\left(a_{1}, \ldots, a_{5}\right)$ are regular closed sets in $\mathbb{R}^{2}$ :

Theorem 15. There is a $\mathcal{B} c^{\circ}$-formula satisfiable over $\mathrm{RC}\left(\mathbb{R}^{2}\right)$, but only by tuples featuring sets with infinitely many components.

Proof. We first write a $\mathcal{C} c^{\circ}$-formula, $\varphi_{\infty}^{*}$ with the required properties, and then show that all occurrences of $C$ in it can be eliminated. Note that $\varphi_{\infty}^{*}$ is not the same as the formula $\varphi_{\infty}^{\circ}$ constructed for the proof of Corollary 6 . As with the proof of Theorem 8, we equivocate between variables and the regions to which they are assigned in some putative interpretation over $\mathrm{RC}\left(\mathbb{R}^{2}\right)$. If $k$ is an integer, $\lfloor k\rfloor$ indicates the value of $k$ modulo 2 .

Let $s_{0}, \ldots, s_{3}, a, b, a_{i, j}$ and $b_{i, j}$ be variables, for $0 \leq i<2,1 \leq j \leq 3$. The constraints

$$
\begin{aligned}
& \operatorname{frame}^{\circ}\left(s_{0}, s_{1}, b, s_{2}, a, s_{3}\right), \\
& \operatorname{stack}^{\circ}\left(s_{0}, b_{1,1}, b_{1,2}, b_{1,3}, b\right), \\
& \bigwedge_{i=0}^{1} \operatorname{stack}^{\circ}\left(b_{i, 2}, a_{i, 1}, a_{i, 2}, a_{i, 3}, a\right), \\
& \bigwedge_{i=0}^{1} \operatorname{stack}^{\circ}\left(a_{\lfloor i-1\rfloor, 2}, b_{i, 1}, b_{i, 2}, b_{i, 3}, b\right)
\end{aligned}
$$

are evidently satisfied by the arrangement of Fig. 11 . Let $\varphi_{\infty}^{*}$ be the conjunction of (33)-(36) as well as formulas

$$
\left(r \cdot r^{\prime}=0\right), \quad \text { for distinct variables } r \text { and } r^{\prime} .
$$




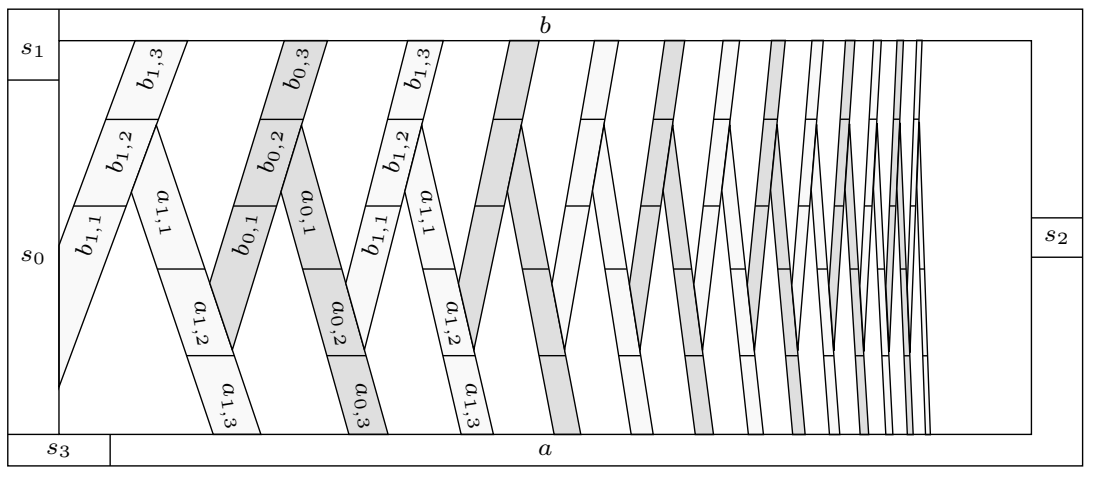

Figure 11: A tuple satisfying [33]-(36): the pattern of components of the $a_{i, j}$ and $b_{i, j}$ repeats forever.

Note that the regions $a_{i, j}$ and $b_{i, j}$ have infinitely many components. We will show that this is true for every satisfying tuple of $\varphi_{\infty}^{*}$.

By (33) and Lemma 12 there is a Jordan curve $\sigma \lambda_{0} \mu_{0}^{-1}$ whose segments are Jordan arcs lying in the respective sets $\left(s_{3}+s_{0}+s_{1}\right)^{\circ},\left(s_{2}+a+s_{3}\right)^{\circ}$ and $\left(s_{1}+b+s_{2}\right)^{\circ}$; see Fig. 12 Note that all points in $s_{0}$ that are on $\sigma \lambda_{0} \mu_{0}^{-1}$ are on $\sigma$. Let $o^{\prime}$ be the common point of $\mu_{0}$ and $\lambda_{0}$ and $\tilde{q}_{1,1} \in \sigma \cap s_{0}^{\circ}$.

A word is required concerning the generality of this and other diagrams in this section. The reader is to imagine the figure drawn on a spherical canvas, of which the sheet of paper or computer screen in front of him is simply a small part. This sphere represents the plane with a 'point' at infinity, under the usual stereographic projection. We do not say where this point at infinity is, other than that it never lies on a drawn arc. In this way, a diagram in which the spherical canvas is divided into $n$ cells represents $n$ different configurations in the plane - one for each of the cells in which the point at infinity may be located. For example, Fig. 12 represents two topologically distinct configurations in $\mathbb{R}^{2}$, and, as such, depicts the $\operatorname{arcs} \sigma, \lambda_{0}$ and $\mu_{0}$ and points $\tilde{q}_{1,1}, o^{\prime}$ in full generality. All diagrams in this proof are to be interpreted in this way. We stress that our 'spherical diagrams' are simply a convenient device for using one drawing to represent several possible configurations in the Euclidean plane: in particular, we are interested only in the satisfiability of $\mathcal{B} c^{\circ}$-formulas over $\mathrm{RC}\left(\mathbb{R}^{2}\right)$, not over the regular closed algebra of any other space!

Let $\tilde{q}_{1,3} \in \mu_{0} \cap b^{\circ}$. By (34) and Lemma 13 , we can connect $\tilde{q}_{1,1}$ to $\tilde{q}_{1,3}$ by a Jordan $\operatorname{arc} \tilde{\beta}_{1,1} \beta_{1,2} \tilde{\beta}_{1,3}$ whose segments lie in the respective sets $\left(s_{0}+b_{1,1}\right)^{\circ}$, $\left(b_{1,1}+b_{1,2}+b_{1,3}\right)^{\circ}$ and $\left(b_{1,3}+b\right)^{\circ}$; see Fig. 13 Let $q_{1,1}$ be the last point on $\tilde{\beta}_{1,1}$ that is on $\sigma$ and let $\beta_{1,1}$ be the final segment of $\tilde{\beta}_{1,1}$ starting at $q_{1,1}$; by Lemma 11 $q_{1,1} \in s_{0}^{\circ}$. Similarly, let $q_{1,3}$ be the first point on $\tilde{\beta}_{1,3}$ that is on $\mu_{0}$ and let $\beta_{1,3}$ be the initial segment of $\tilde{\beta}_{1,3}$ ending at $q_{1,3}$; by Lemma 11 . $q_{1,3} \in b^{\circ}$. Hence, the arc $\beta_{1,1} \beta_{1,2} \beta_{1,3}$ lies in exactly one of the regions bounded by $\sigma \lambda_{0} \mu_{0}^{-1}$ : for reasons that will emerge in the course of the proof, we denote 


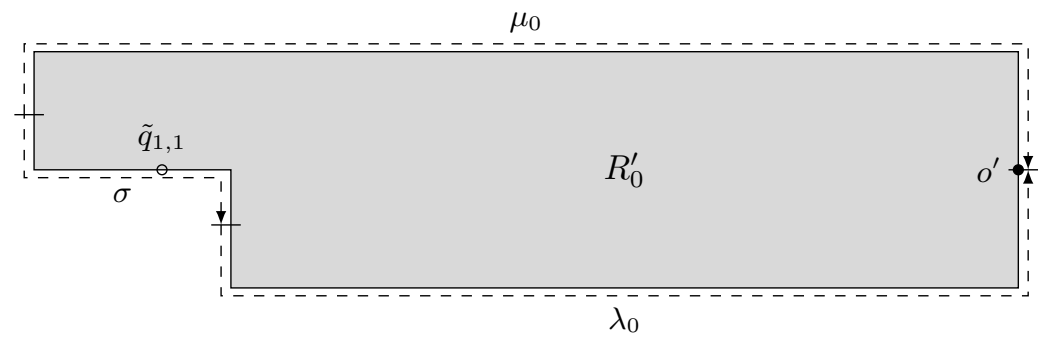

Figure 12: The $\operatorname{arcs} \sigma, \mu_{0}$ and $\lambda_{0}$.

that region $R_{0}^{\prime}$. Now, $\beta_{1,1} \beta_{1,2} \beta_{1,3}$ divides $R_{0}^{\prime}$ into two sub-regions: we denote the sub-region whose boundary is disjoint from $a$ by $S_{1}$, and the other sub-region by $S_{1}^{\prime}$. Let $\mu_{1}=\beta_{1,3} \mu_{0}\left[q_{1,3}, o^{\prime}\right] \subseteq\left(b_{1,3}+s_{1}+b+s_{2}\right)^{\circ}$. The arc $\beta_{1,2}$ contains a point $\tilde{p}_{1,1} \in b_{1,2}^{\circ}$; moreover, all points of $\beta_{1,1} \beta_{1,2} \beta_{1,3}$ in $b_{1,2}^{\circ}$ lie on $\beta_{1,2}$.

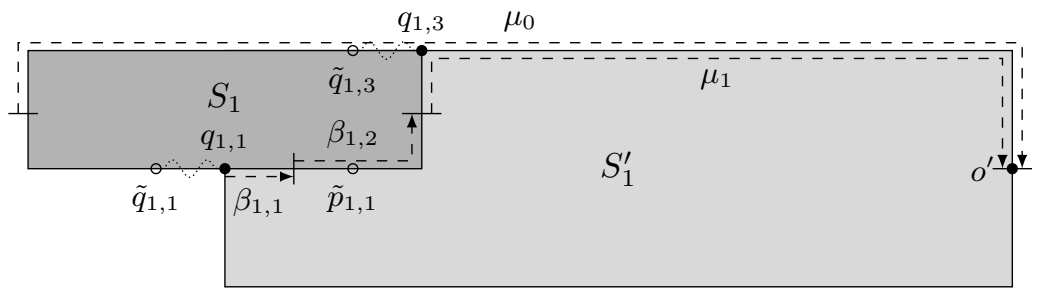

Figure 13: The regions $S_{1}$ and $S_{1}^{\prime}$.

We will now construct a cross-cut $\alpha_{1,1} \alpha_{1,2} \alpha_{1,3}$ in $S_{1}^{\prime}$. Let $\tilde{p}_{1,3}$ be a point in $\lambda_{0} \cap a^{\circ}$. By (35) and Lemma 13 , we can connect $\tilde{p}_{1,1}$ to $\tilde{p}_{1,3}$ by a Jordan $\operatorname{arc} \tilde{\alpha}_{1,1} \alpha_{1,2} \tilde{\alpha}_{1,3}$ whose segments lie in the respective sets $\left(b_{1,2}+a_{1,1}\right)^{\circ}$, $\left(a_{1,1}+a_{1,2}+a_{1,3}\right)^{\circ}$ and $\left(a_{1,3}+a\right)^{\circ}$; see Fig. 14 . Let $p_{1,1}$ be the last point on $\tilde{\alpha}_{1,1}$ that is on $\beta_{1,2}$ and let $\alpha_{1,1}$ be the final segment of $\tilde{\alpha}_{1,1}$ starting at $p_{1,1}$; by Lemma 11, $p_{1,1} \in b_{1,2}^{\circ}$. Similarly, let $p_{1,3}$ be the first point on $\tilde{\alpha}_{1,3}$ that is on $\lambda_{0}$ and let $\alpha_{1,3}$ be the initial segment of $\tilde{\alpha}_{1,3}$ ending at $p_{1,3}$; by Lemma 11. $p_{1,3} \in a^{\circ}$. Since $\alpha_{1,1} \alpha_{1,2} \alpha_{1,3}$ does not intersect the boundaries of $S_{1}$ and $S_{1}^{\prime}$ except at its endpoints, it is a cross-cut in one of these regions. Moreover, that region has to be $S_{1}^{\prime}$ since the boundary of $S_{1}$ is disjoint from $a$. So, $\alpha_{1,1} \alpha_{1,2} \alpha_{1,3}$ divides $S_{1}^{\prime}$ into two sub-regions: we denote the sub-region whose boundary is disjoint from $b$ by $R_{1}$, and the other sub-region by $R_{1}^{\prime}$. Let $\lambda_{1}=\alpha_{1,3} \lambda_{0}\left[p_{1,3}, o^{\prime}\right] \subseteq\left(a_{1,3}+s_{3}+a+s_{2}\right)^{\circ}$. The arc $\alpha_{1,2}$ contains a point $\tilde{q}_{2,1} \in a_{1,2}^{\circ} ;$ moreover, all points of $\alpha_{1,1} \alpha_{1,2} \alpha_{1,3}$ in $a_{1,2}^{\circ}$ lie on $\alpha_{1,2}$.

We can now forget about the region $S_{1}$, and start constructing a cross-cut $\beta_{2,1} \beta_{2,2} \beta_{2,3}$ in $R_{1}^{\prime}$. As before, let $\tilde{q}_{2,3} \in \mu_{1} \cap b^{\circ}$. Then there is a Jordan arc $\tilde{\beta}_{2,1} \beta_{2,2} \tilde{\beta}_{2,3}$ connecting $\tilde{q}_{2,1}$ to $\tilde{q}_{2,3}$ such that its segments are contained in the respective sets $\left(a_{1,2}+b_{0,1}\right)^{\circ},\left(b_{0,1}+b_{0,2}+b_{0,3}\right)^{\circ}$ and $\left(b_{0,3}+b\right)^{\circ}$. As before, we 


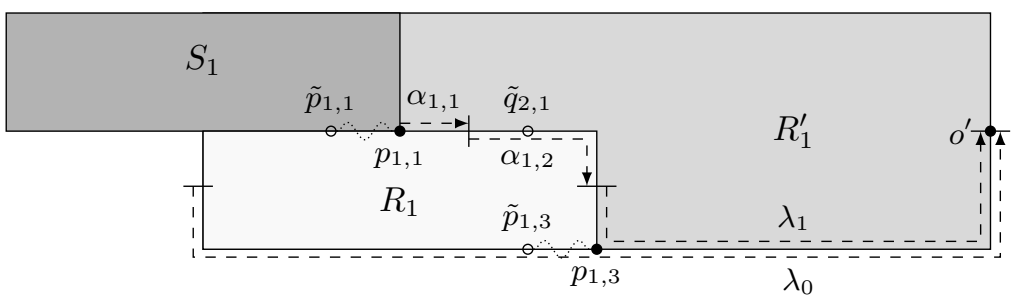

Figure 14: The regions $S_{1}, R_{1}$ and $R_{1}^{\prime}$.

choose $\beta_{2,1} \subseteq \tilde{\beta}_{2,1}$ and $\beta_{2,3} \subseteq \tilde{\beta}_{2,3}$ so that the Jordan $\operatorname{arc} \beta_{2,1} \beta_{2,2} \beta_{2,3}$ without its endpoints is disjoint from the boundaries of $R_{1}$ and $R_{1}^{\prime}$. Hence $\beta_{2,1} \beta_{2,2} \beta_{2,3}$ has to be a cross-cut in $R_{1}$ or $R_{1}^{\prime}$, and since the boundary of $R_{1}$ is disjoint from $b$ it has to be a cross-cut in $R_{1}^{\prime}$. So, $\beta_{2,1} \beta_{2,2} \beta_{2,3}$ separates $R_{1}^{\prime}$ into two regions $S_{2}$ and $S_{2}^{\prime}$ so that the boundary of $S_{2}$ is disjoint from $a$. Let $\mu_{2}=\beta_{2,3} \mu_{1}\left[q_{2,3}, o^{\prime}\right] \subseteq$ $\left(b_{0,3}+b_{1,3}+s_{1}+b+s_{2}\right)^{\circ}$. Now, we can ignore the region $R_{1}$, and reasoning as before we can construct a cross-cut $\alpha_{2,1} \alpha_{2,2} \alpha_{2,3}$ in $S_{2}^{\prime}$ dividing it into two sub-regions $R_{2}$ and $R_{2}^{\prime}$.

Evidently, this process continues forever: $R_{i-1}^{\prime}$ is divided into $S_{i}$ and $S_{i}^{\prime}$ and $S_{i}^{\prime}$ is divided into $R_{i}$ and $R_{i}^{\prime}$. Now, the boundary of $S_{i}$ contains the arc $\beta_{i, 2}$, whence the interior of $S_{i}$ contains points of $b_{\lfloor i\rfloor, 2}$. On the other hand, $S_{i}$ certainly lies outside $S_{i+1}^{\prime}$; moreover, $\delta S_{i+1}^{\prime}$ is a subset of $\alpha_{i, 2} \cup \beta_{i+1,1} \cup \beta_{i+1,2} \cup$ $\mu_{i+1} \cup \lambda_{i}$, whence

$$
\begin{aligned}
& \delta S_{i+1}^{\prime} \subseteq\left(a_{\lfloor i\rfloor, 1}+\right.\left.a_{\lfloor i\rfloor, 2}+a_{\lfloor i\rfloor, 3}\right)^{\circ} \cup\left(a_{\lfloor i\rfloor, 2}+b_{\lfloor i+1\rfloor, 1}\right)^{\circ} \cup \\
&\left(b_{\lfloor i+1\rfloor, 1}+b_{\lfloor i+1\rfloor, 2}+b_{\lfloor i+1\rfloor, 3}\right)^{\circ} \cup \\
&\left(b_{0,3}+b_{1,3}+b+s_{1}+s_{2}\right)^{\circ} \cup\left(a_{0,3}+a_{1,3}+a+s_{2}+s_{3}\right)^{\circ} .
\end{aligned}
$$

Hence $\delta S_{i+1}^{\prime}$ contains no points of $b_{\lfloor i\rfloor, 2}$. Yet $S_{i+1}^{\prime}$ evidently includes all the regions $S_{i+2 k}$ for all $k \geq 1$, each of which contains points of $b_{\lfloor i\rfloor, 2}$. It follows that $b_{\lfloor i\rfloor, 2}$ has infinitely many components.

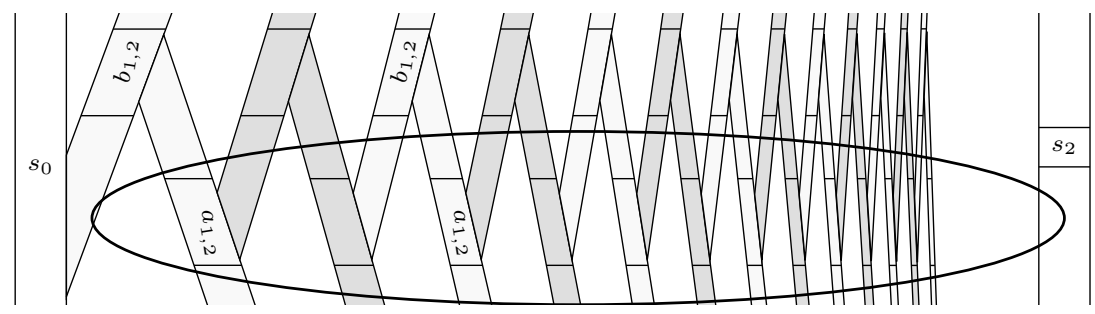

Figure 15: Separating $a_{1,2}$ from $b_{1,2}$ by a Jordan curve.

So far we know that the $\mathcal{C} c^{\circ}$-formula $\varphi_{\infty}^{*}$ forces infinitely many components. Now we replace every conjunct in $\varphi_{\infty}^{*}$ of the form $\neg C\left(s_{1}, s_{2}\right)$ by $\operatorname{K5m}(\vec{r}) \wedge\left(s_{1} \leq\right.$ 
$\left.r_{1}\right) \wedge\left(s_{2} \leq r_{2}\right)$, where $\vec{r}$ is a vector of fresh variables. By Lemma $14(i)$, the resulting formula entails $\varphi_{\infty}^{*}$. Conversely, to show that the formula is satisfiable, we apply Lemma 14 ( $i i)$ : it suffices to separate every pair of disjoint regions in Fig. 11 by a Jordan curve. Such a Jordan curve is shown in Fig. 15 for $b_{1,2}$ and $a_{1,2}$. Other pairs of disjoint regions are treated analogously.

\subsection{Undecidability in the plane}

We now return to the question of decidability. We know from Sec. 4 that $\operatorname{Sat}\left(\mathcal{L}, \operatorname{RCP}\left(\mathbb{R}^{2}\right)\right)$ is undecidable, where $\mathcal{L}$ is any of the languages $\mathcal{B} c, \mathcal{C} c$ or $\mathcal{C} c^{\circ}$. We proceed to establish the undecidability of the problems $\operatorname{Sat}\left(\mathcal{L}, \operatorname{RC}\left(\mathbb{R}^{2}\right)\right)$, where $\mathcal{L}$ is any of the languages $\mathcal{B} c, \mathcal{B} c^{\circ}, \mathcal{C} c$ or $\mathcal{C} c^{\circ}$, and also of the problem $\operatorname{Sat}\left(\mathcal{B} c^{\circ}, \operatorname{RCP}\left(\mathbb{R}^{2}\right)\right)$. Most of the techniques required have been rehearsed in the proof of Theorem 15. However, we face a new difficulty. In the language $\mathcal{B} c^{\circ}$, we can say that the interior of a region (rather than merely the region itself) is connected. Since, for open sets, connectedness implies arc-connectedness, we were able, in the proof of Theorem 15, to write formulas enforcing various arrangements of Jordan arcs in the plane. When dealing with $\mathcal{B} c$ and $\mathcal{C} c$, however, we can speak merely of the connectedness of a region (rather than of its interior), which, for elements of $\mathrm{RC}\left(\mathbb{R}^{2}\right)$ does not imply arc-connectedness; this complicates the business of enforcing the requisite arrangements of Jordan arcs.

To overcome this difficulty, we employ the technique of 'wrapping' a region inside two bigger ones. If $a$ and $b$ are regions such that $\neg C(a,-b)$, we write $a \ll b$ (pronounced: $a$ is right inside $b$ ). Let us say that a 3-region is a triple $\mathfrak{a}=(a, \dot{a}, \ddot{a})$ of elements of $\mathrm{RC}\left(\mathbb{R}^{2}\right)$ such that $0 \neq \ddot{a} \ll \dot{a} \ll a$. It helps to think of $\mathfrak{a}=(a, \dot{a}, \ddot{a})$ as consisting of a kernel, $\ddot{a}$, encased in two protective layers: an inner shell, $\dot{a}$ and an outer shell, $a$. As a simple example, consider the sequence of 3-regions $\mathfrak{a}_{1}, \mathfrak{a}_{2}, \mathfrak{a}_{3}$ depicted in Fig. 16, where the kernels form a sequence of externally touching polygons. When describing arrangements of 3-regions,

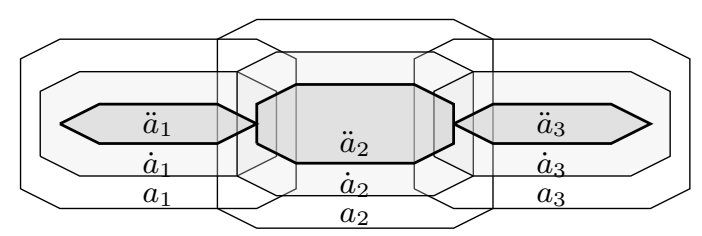

Figure 16: A chain of 3-regions satisfying $\operatorname{stack}\left(\mathfrak{a}_{1}, \mathfrak{a}_{2}, \mathfrak{a}_{3}\right)$.

we use the variable $\mathfrak{r}$ for the triple of variables $(r, \dot{r}, \ddot{r})$, taking the following conjuncts to be implicit:

$$
(\ddot{r} \neq 0) \wedge(\ddot{r} \ll \dot{r}) \wedge(\dot{r} \ll r) .
$$

In the sequel, when depicting arrangements of 3-regions, we standardly draw only the kernels of these 3-regions, leaving the reader to imagine the encasing layers of shell. (This is simply to reduce diagrammatic clutter.) 
For $n \geq 2$, define the formula

$$
\operatorname{stack}\left(\mathfrak{r}_{1}, \ldots, \mathfrak{r}_{n}\right)=\bigwedge_{i=1}^{n-1} c\left(\dot{r}_{i}+\ddot{r}_{i+1}+\cdots+\ddot{r}_{n}\right) \wedge c\left(\dot{r}_{n}\right) \wedge \bigwedge_{\substack{1 \leq i, j \leq n \\ j-i>1}} \neg C\left(r_{i}, r_{j}\right) .
$$

(Observe that the term $c\left(\dot{r}_{i}+\ddot{r}_{i+1}+\cdots+\ddot{r}_{n}\right)$ features the inner shell of $\mathfrak{r}_{1}$, and the kernels of $\mathfrak{r}_{2}, \ldots, \mathfrak{r}_{n}$.) Thus, the triple of 3-regions $\left(\mathfrak{a}_{1}, \mathfrak{a}_{2}, \mathfrak{a}_{3}\right)$ in Fig. 16 satisfies $\operatorname{stack}\left(\mathfrak{r}_{1}, \mathfrak{r}_{2}, \mathfrak{r}_{3}\right)$. This formula allows us to construct sequences of arcs with useful properties.

Lemma 16. Fix $n \geq 2$, and let $\mathfrak{a}_{1}, \ldots, \mathfrak{a}_{n}$ be a tuple of 3-regions satisfying $\operatorname{stack}\left(\mathfrak{r}_{1}, \ldots, \mathfrak{r}_{n}\right)$. Then, for every point $p_{0} \in \dot{a}_{1}$ and every point $p_{n} \in \ddot{a}_{n}$, there exist points $p_{1}, \ldots, p_{n-1}$ and Jordan arcs $\alpha_{1}, \ldots, \alpha_{n}$ such that: $(i) \alpha=\alpha_{1} \cdots \alpha_{n}$ is a Jordan arc from $p_{0}$ to $p_{n} ;$ (ii) $p_{i} \in \dot{a}_{i+1} \cap \alpha_{i}$, for all $1 \leq i<n$; and (iii) $\alpha_{i} \subseteq a_{i}$, for all $1 \leq i \leq n$.

Proof. Let $v_{0}=p_{0}$. Since $v_{0} \in a_{1}^{\circ}, p_{n} \in \dot{a}_{n}^{\circ}$ and $\dot{a}_{1}+\ddot{a}_{2}+\cdots+\ddot{a}_{n}$ is connected, we see that $v_{0}$ and $p_{0}$ lie in the same component of $\left(a_{1}+\dot{a}_{2}+\cdots+\dot{a}_{n}\right)^{\circ}$. So let $\beta_{1}$ be a Jordan arc connecting $v_{0}$ to $p_{n}$ in $\left(a_{1}+\dot{a}_{2}+\cdots+\dot{a}_{n}\right)^{\circ}$. Since $a_{1}$ is disjoint from all the $a_{i}$ except $a_{2}$, let $p_{1}$ be the first point of $\beta_{1}$ lying in $\dot{a}_{2}$, so $\beta_{1}\left[v_{0}, p_{1}\right] \subseteq a_{1}^{\circ} \cup\left\{p_{1}\right\}$, i.e., the arc $\beta_{1}\left[v_{0}, p_{1}\right]$ is either included in $a_{1}^{\circ}$, or is an end-cut of $a_{1}^{\circ}$. (We do not rule out $v_{0}=p_{1}$.) Similarly, let $\beta_{2}^{\prime}$ be a Jordan arc connecting $p_{1}$ to $p_{n}$ in $\left(a_{2}+\dot{a}_{3}+\cdots+\dot{a}_{n}\right)^{\circ}$, and let $q_{1}$ be the last point of $\beta_{2}^{\prime}$ lying on $\beta_{1}\left[v_{0}, p_{1}\right]$. If $q_{1}=p_{1}$, then set $v_{1}=p_{1}, \alpha_{1}=\beta_{1}\left[v_{0}, p_{1}\right]$, and $\beta_{2}=\beta_{2}^{\prime}$, so that the endpoints of $\beta_{2}$ are $v_{1}$ and $p_{n}$. Otherwise, we have $q_{1} \in a_{1}^{\circ}$. We can now construct an arc $\gamma_{1} \subseteq a_{1}^{\circ} \cup\left\{p_{1}\right\}$ from $p_{1}$ to a point $v_{1}$ on $\beta_{2}^{\prime}\left[q_{1}, p_{n}\right]$, such that $\gamma_{1}$ intersects $\beta_{1}\left[v_{0}, p_{1}\right]$ and $\beta_{2}^{\prime}\left[q_{1}, p_{n}\right]$ only at its endpoints, $p_{1}$ and $v_{1}$; see Fig. 17. Let $\alpha_{1}=\beta_{1}\left[v_{0}, p_{1}\right] \gamma_{1}$, and let $\beta_{2}=\beta_{2}^{\prime}\left[v_{1}, p_{n}\right]$.

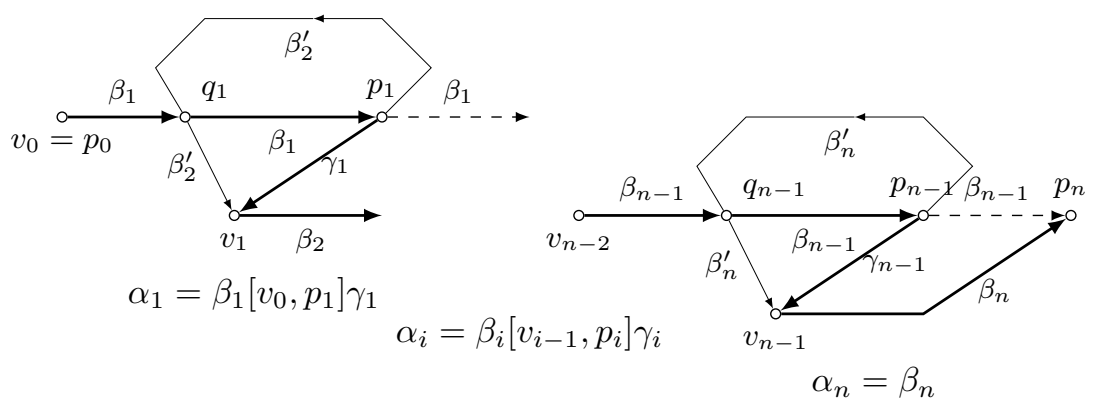

Figure 17: Proof of Lemma 16 .

Since $\beta_{2}$ contains a point $p_{2} \in \dot{a}_{3}$, we may iterate this procedure, obtaining $\alpha_{2}, \alpha_{3}, \ldots \alpha_{n-1}, \beta_{n}$. We remark that $\alpha_{i}$ and $\alpha_{i+1}$ have a single point of contact by construction, while $\alpha_{i}$ and $\alpha_{j}(i<j-1)$ are disjoint by the constraint $\neg C\left(a_{i}, a_{j}\right)$. Finally, we let $\alpha_{n}=\beta_{n}$; see Fig. 17 . 
In fact, we can add a 'switch' to the formula stack $\left(\mathfrak{r}_{1}, \ldots, \mathfrak{r}_{n}\right)$, in the following sense. Recall from Sec. 2 that if $a, a_{0}, \ldots, a_{n-1}$ are regions satisfying $\operatorname{colour}\left(r ; r_{0}, \ldots, r_{n-1}\right)$, then every connected subset of $a$-and in particular, any component of $a$-is included in exactly one of the $a_{0}, \ldots, a_{n-1}$. Let $z$ be a variable, and consider what happens when we replace the variable $\dot{r}_{1}$ in $\operatorname{stack}\left(\mathfrak{r}_{1}, \ldots, \mathfrak{r}_{n}\right)$ by the term $(-z) \cdot \dot{r}_{1}$, and add the conjunct colour $\left(\dot{r}_{1} ; z,-z\right)$. The result is

$$
\begin{array}{r}
\operatorname{stack}_{z}\left(\mathfrak{r}_{1}, \ldots, \mathfrak{r}_{n}\right)=\operatorname{colour}\left(\dot{r}_{1} ; z,-z\right) \wedge c\left(\left((-z) \cdot \dot{r}_{1}\right)+\ddot{r}_{2}+\cdots+\ddot{r}_{n}\right) \wedge \\
\bigwedge_{i=2}^{n-1} c\left(\dot{r}_{i}+\ddot{r}_{i+1}+\cdots+\ddot{r}_{n}\right) \wedge c\left(\dot{r}_{n}\right) \wedge \bigwedge_{\substack{1 \leq i, j \leq n \\
j-i>1}} \neg C\left(r_{i}, r_{j}\right) .
\end{array}
$$

Now let $\mathfrak{a}_{1}, \ldots, \mathfrak{a}_{n}$ be 3 -regions and $d$ a region satisfying $\operatorname{stack}_{d}\left(\mathfrak{r}_{1}, \ldots, \mathfrak{r}_{n}\right)$. The first conjunct of the formula ensures that any component of $\dot{a}_{1}$ is either included in $d$ or included in $-d$. The remaining conjuncts then have the same effect as $\operatorname{stack}\left(\mathfrak{r}_{1}, \ldots, \mathfrak{r}_{n}\right)$-but only for those components of $\dot{a}_{1}$ included in $-d$. That is, if $p \in(-d) \cdot \dot{a}_{1}$, we can find an arc $\alpha_{1} \cdots \alpha_{n}$ starting at $p$, with the properties of Lemma 16. However, if $p \in d \cdot \dot{a}_{1}$, no such arc need exist. Thus, the variable $z$ functions so as to 'de-activate' $\operatorname{stack}_{z}\left(\mathfrak{r}_{1}, \ldots, \mathfrak{r}_{n}\right)$ when we are dealing with a component of $\dot{r}_{1}$ satisfying $\dot{r}_{1} \leq z$.

As a further application of Lemma 16, consider the formula

$$
\begin{aligned}
\text { frame }\left(\mathfrak{r}_{0}, \ldots, \mathfrak{r}_{n}\right)=\operatorname{stack}\left(\mathfrak{r}_{0}, \ldots, \mathfrak{r}_{n-1}\right) & \wedge \neg C\left(r_{n}, r_{1}+\cdots+r_{n-2}\right) \wedge \\
c\left(\dot{r}_{n}\right) & \wedge\left(\dot{r}_{0} \cdot \dot{r}_{n} \neq 0\right) \wedge\left(\ddot{r}_{n-1} \cdot \dot{r}_{n} \neq 0\right) .
\end{aligned}
$$

This formula allows us to construct Jordan curves in the plane, in the following sense:

Lemma 17. Fix $n \geq 3$, and let $\mathfrak{a}_{0}, \ldots, \mathfrak{a}_{n}$ be a tuple of 3-regions satisfying frame $\left(\mathfrak{r}_{0}, \ldots, \mathfrak{r}_{n}\right)$. Then there exist Jordan arcs $\gamma_{0}, \ldots, \gamma_{n}$ such that $\gamma_{0} \cdots \gamma_{n}$ is a Jordan curve and $\gamma_{i} \subseteq a_{i}$, for all $0 \leq i \leq n$.

Proof. By Lemma 16, let $\alpha_{0}, \gamma_{1}, \ldots, \gamma_{n-2}, \alpha_{n-1}$ be Jordan arcs in the respective regions $a_{0}, \ldots, a_{n-1}$ such that $\alpha_{0} \cdots \alpha_{n-1}$ is a Jordan arc connecting a point $\tilde{p} \in \dot{a}_{0} \cdot \dot{a}_{n}$ to a point $\tilde{q} \in \ddot{a}_{n-1} \cdot \dot{a}_{n}$; see Fig. 18. Because $\dot{a}_{n}$ is a connected subset

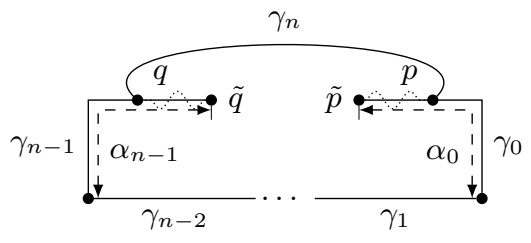

Figure 18: Establishing a Jordan curve. 
of the interior of $a_{n}$, let $\alpha_{n} \subseteq a_{n}^{\circ}$ be an arc connecting $\tilde{p}$ and $\tilde{q}$. Note that $\alpha_{n}$ does not intersect $\alpha_{i}$, for $1 \leq i \leq n-2$. Let $p$ be the last point on $\alpha_{0}$ that is on $\alpha_{n}$ (possibly $\tilde{p}$ ), and $q$ be the first point on $\alpha_{n-1}$ that is on $\alpha_{n}$ (possibly $\tilde{q}$ ). Let $\gamma_{0}$ be the final segment of $\alpha_{0}$ starting at $p$ and let $\gamma_{n-1}$ be the initial segment of $\alpha_{n-1}$ ending at $q$. Finally, let $\gamma_{n}=\alpha_{n}[p, q]$ or $\gamma_{n}=\alpha_{n}[q, p]$, depending on whether $p$ or $q$ is encountered first on $\alpha_{n}$. Then the $\operatorname{arcs} \gamma_{i}, 0 \leq i \leq n$, are as required.

We are now ready to prove the main result of this section. Again, recall that if $a, a_{0}, \ldots, a_{n-1}$ are regions satisfying $\operatorname{colour}\left(r ; r_{0}, \ldots, r_{n-1}\right)$, then every connected subset of $a$-and in particular, any Jordan arc $\alpha \subseteq a$-is included in exactly one of the $a_{i}$, for $0 \leq i<n$. In this case, it is sometimes helpful to think of $\alpha$ as being 'labelled' by a letter of the alphabet $a_{0}, \ldots, a_{n-1}$.

Theorem 18. The problems $\operatorname{Sat}\left(\mathcal{C} c, \mathrm{RC}\left(\mathbb{R}^{2}\right)\right)$ and $\operatorname{Sat}\left(\mathcal{C} c, \mathrm{RCP}\left(\mathbb{R}^{2}\right)\right)$ are r.e.hard.

Proof. Again, we proceed via a reduction of the Post correspondence problem (PCP), constructing, for any instance $\mathrm{W}$, a formula $\psi_{\mathrm{W}}$ with the property that the following are equivalent: $(i) \mathrm{W}$ is positive; $(i i) \psi_{\mathrm{W}}$ is satisfiable over $\operatorname{RCP}\left(\mathbb{R}^{2}\right) ;(i i i) \psi_{\mathrm{W}}$ is satisfiable over $\mathrm{RC}\left(\mathbb{R}^{2}\right)$. This establishes the theorem. As with the proofs of Theorems 8 and 15 , we equivocate between variables and the regions to which they are assigned in some putative interpretation over $\operatorname{RC}\left(\mathbb{R}^{2}\right)$. In this proof, if $k$ is an integer, $\lfloor k\rfloor$ indicates the value of $k$ modulo 3 . The proof proceeds in six stages.

\begin{tabular}{|c|c|c|c|c|c|}
\hline $\mathfrak{s}_{5}^{\prime}$ & 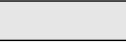 & \multicolumn{4}{|l|}{$\mathfrak{s}_{6}^{\prime}$} \\
\hline$\frac{\mathfrak{s}_{4}^{\prime}}{\prime^{\prime}}$ & \multirow{2}{*}{$\mathfrak{d}_{0}$} & & \multirow{2}{*}{$\mathfrak{d}_{6}$} & & $\mathfrak{s}_{7}^{\prime}$ \\
\hline$\frac{\mathfrak{s}_{2}}{\mathfrak{s}^{\prime}}$ & & & & & $\mathfrak{s}_{8}^{\prime}$ \\
\hline$\overline{\mathfrak{s}_{0}}$ & $\mathfrak{d}_{1} \mid \mathfrak{d}_{2}$ & $\mathfrak{d}_{3}$ & $\mathfrak{d}_{4}$ & $\mathfrak{d}_{5}$ & $\mathfrak{s}_{9}$ \\
\hline $\mathfrak{s}_{1}$ & & & & & $\mathfrak{s}_{8}$ \\
\hline$\underline{\mathfrak{s}_{2}}$ & & & & & \\
\hline $\mathfrak{s}_{3}$ & & & & & $\mathfrak{5}$ \\
\hline $\mathfrak{s}_{4}$ & & & & & \\
\hline $\mathfrak{s}_{5}$ & & $\mathfrak{s}_{6}$ & & & \\
\hline
\end{tabular}

Figure 19: A tuple of 3-regions satisfying (38)-40) (showing kernels only). The 3 -regions $\mathfrak{s}_{0}$ and $\mathfrak{s}_{9}$ are right inside $\mathfrak{d}_{0}$ and $\mathfrak{d}_{6}$, respectively, as specified by (39).

Stage 1. In the first stage, we define an assemblage of arcs that will serve as scaffolding for the ensuing construction. Consider the arrangement of polygonal 3-regions depicted in Fig. 19, assigned to the 3-region variables $\mathfrak{s}_{0}, \ldots, \mathfrak{s}_{9}$, $\mathfrak{s}_{8}^{\prime}, \ldots, \mathfrak{s}_{1}^{\prime}, \mathfrak{d}_{0}, \ldots, \mathfrak{d}_{6}$ as indicated. (Note that we have here followed the convention of depicting only the kernels of 3-regions.) 
It is easy to verify that this arrangement can be made to satisfy the following formulas:

$$
\begin{aligned}
& \text { frame }\left(\mathfrak{s}_{0}, \mathfrak{s}_{1}, \ldots, \mathfrak{s}_{8}, \mathfrak{s}_{9}, \mathfrak{s}_{8}^{\prime}, \ldots, \mathfrak{s}_{1}^{\prime}\right) \\
& \left(s_{0} \leq \dot{d}_{0}\right) \wedge\left(s_{9} \leq \ddot{d}_{6}\right) \\
& \operatorname{stack}\left(\mathfrak{d}_{0}, \ldots, \mathfrak{d}_{6}\right)
\end{aligned}
$$

And obviously, the arrangement can be made to satisfy any formula

$$
\neg C\left(r, r^{\prime}\right),
$$

for which the corresponding 3-regions $\mathfrak{r}$ and $\mathfrak{r}^{\prime}$ are drawn as not being in contact. (Remember, $r$ is the outer shell of the 3-region $\mathfrak{r}$, and similarly for $r^{\prime}$; so we must take these shells to hug the kernels depicted in Fig. 19 quite closely.) Thus, for example, 41) includes $\neg C\left(s_{0}, d_{1}\right)$, but not $\neg C\left(s_{0}, d_{0}\right)$ or $\neg C\left(d_{0}, d_{1}\right)$.

Now suppose $\mathfrak{s}_{0}, \ldots, \mathfrak{s}_{9}, \mathfrak{s}_{8}^{\prime}, \ldots, \mathfrak{s}_{1}^{\prime}, \mathfrak{d}_{0}, \ldots, \mathfrak{d}_{6}$ is any collection of 3 -regions (not necessarily polygonal) satisfying (38)-41). By Lemma 17 and (38), let $\gamma_{0}, \ldots, \gamma_{9}, \gamma_{8}^{\prime}, \ldots, \gamma_{1}^{\prime}$ be Jordan arcs included in the respective regions $s_{0}, \ldots, s_{9}, s_{8}^{\prime}, \ldots, s_{1}^{\prime}$, such that $\Gamma=\gamma_{0} \cdots \gamma_{9} \cdot \gamma_{8}^{\prime} \cdots \gamma_{1}^{\prime}$ is a Jordan curve (note that $\gamma_{i}^{\prime}$ and $\gamma_{i}$ have opposite directions). We select points $\tilde{o}$ on $\gamma_{0}$ and $\tilde{o}^{\prime}$ on $\gamma_{9}$; see Fig. 20 By (39), $\tilde{o} \in \dot{d}_{0}$ and $\tilde{o}^{\prime} \in \ddot{d}_{6}$. By Lemma 16 and $[40)$, let $\tilde{\chi}_{1}, \chi_{2}, \tilde{\chi}_{3}$ be Jordan arcs in the respective regions

$$
\left(d_{0}+d_{1}\right), \quad\left(d_{2}+d_{3}+d_{4}\right), \quad\left(d_{5}+d_{6}\right)
$$

such that $\tilde{\chi}_{1} \chi_{2} \tilde{\chi}_{3}$ is a Jordan arc from $\tilde{o}$ to $\tilde{o}^{\prime}$. Let $o$ be the last point of $\tilde{\chi}_{1}$ lying on $\Gamma$, and let $\chi_{1}$ be the final segment of $\tilde{\chi}_{1}$, starting at $o$. Let $o^{\prime}$ be the first point of $\tilde{\chi}_{3}$ lying on $\Gamma$, and let $\chi_{3}$ be the initial segment of $\tilde{\chi}_{3}$, ending at $o^{\prime}$. By (41), we see that the arc $\chi_{1} \chi_{2} \chi_{3}$ intersects $\Gamma$ only in its endpoints, and is thus a chord of $\Gamma$, as shown in Fig. 20 .

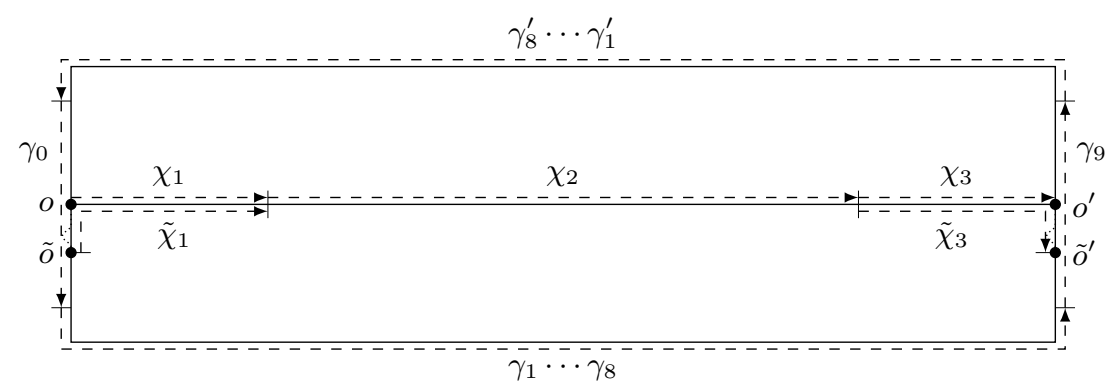

Figure 20: The $\operatorname{arcs} \gamma_{0}, \ldots, \gamma_{9}, \gamma_{8}^{\prime}, \ldots, \gamma_{1}^{\prime}$ and $\chi_{1}, \chi_{2}, \chi_{3}$.

As before, we treat these diagrams as being drawn on a spherical canvas. For ease of reference, we refer to the two rectangles in Fig. 20 as the 'upper window' 
and 'lower window', it being understood that these are simply handy labels: in particular, either (but not both) of these 'windows' may be unbounded.

Stage 2. In this stage, we construct a sequence of triples $\left(\zeta_{i}, \eta_{i}, \kappa_{i}\right)$ of arcs of indeterminate length $n \geq 1$, such that the members of the former sequence all lie in the lower window. (Recall that $\lfloor k\rfloor$ denotes $k$ modulo 3 ). Let $\mathfrak{a}, \mathfrak{b}, \mathfrak{z}, \mathfrak{a}_{i, j}$ and $\mathfrak{b}_{i, j}(0 \leq i<3,1 \leq j \leq 6)$ be 3 -region variables, and consider the formulas

$$
\begin{aligned}
& \left(s_{6} \leq \ddot{a}\right) \wedge\left(s_{6}^{\prime} \leq \ddot{b}\right) \wedge\left(s_{3} \leq \dot{a}_{0,3}\right), \\
& \bigwedge_{i=0}^{2} \operatorname{stack}_{\dot{z}}\left(\mathfrak{a}_{\lfloor i-1\rfloor, 3}, \mathfrak{b}_{i, 1}, \ldots, \mathfrak{b}_{i, 6}, \mathfrak{b}\right), \\
& \bigwedge_{i=0}^{2} \operatorname{stack}\left(\mathfrak{b}_{i, 3}, \mathfrak{a}_{i, 1}, \ldots, \mathfrak{a}_{i, 6}, \mathfrak{a}\right) .
\end{aligned}
$$

\begin{tabular}{|c|c|c|c|c|c|c|c|c|c|c|c|c|}
\hline & \multicolumn{12}{|c|}{$\mathfrak{s}_{6}^{\prime}$} \\
\hline & \multirow{3}{*}{$\mathfrak{b}$} & $\mathfrak{b}_{1,6}$ & & & & & $\mathfrak{b}_{2,6}$ & & & & $\mathfrak{b}_{0,6}$ & \\
\hline & & $\mathfrak{b}_{1,5}$ & & & & & $\mathfrak{b}_{2,5}$ & \multicolumn{3}{|c|}{$\mathfrak{d}_{3}$} & $\mathfrak{b}_{0,5}$ & \\
\hline & & $\mathfrak{b}_{1,4}$ & & & & & $\mathfrak{b}_{2,4}$ & & & & \begin{tabular}{|l|}
$\mathfrak{b}_{0,4}$ \\
\end{tabular} & \\
\hline $\mathfrak{s}_{3}$ & $\mathfrak{b}_{1,1} \mid \mathfrak{b}_{1,2}$ & $\mathfrak{b}_{1,3}$ & $\mathfrak{a}_{1,1}$ & $\mathfrak{a}_{1,2}$ & $\mathfrak{a}_{1,3}$ & $\left|\mathfrak{b}_{2,1}\right| \mathfrak{b}_{2,2}$ & $\mathfrak{b}_{2,3}$ & $\mathfrak{a}_{2,1} \mid \mathfrak{a}_{2,2}$ & $\mathfrak{a}_{2,3}$ & $\mathfrak{b}_{0,1} \mid \mathfrak{b}_{0,2}$ & $\mathfrak{b}_{0,3}$ & $\mathfrak{a}_{0,1} \mid \mathfrak{a}$ \\
\hline & & & & & $\mathfrak{a}_{1,4}$ & & & & $\mathfrak{a}_{2,4}$ & & & \\
\hline & & & & & $\mathfrak{a}_{1,5}$ & & & & $\mathfrak{a}_{2,5}$ & & & \\
\hline & & & & & $\mathfrak{a}_{1,6}$ & & & & $\mathfrak{a}_{2,6}$ & & & \\
\hline & & & & $\mathfrak{s}_{6}$ & & & & & & & & \\
\hline
\end{tabular}

The arrangement of polygonal 3-regions depicted in Fig. 21 (with $\mathfrak{z}$ assigned appropriately) is one such satisfying assignment. We stipulate that (41) applies

Figure 21: A tuple of 3-regions satisfying (42)-(44) (showing kernels only). The arrangement of components of the $\mathfrak{a}_{i, j}$ and $\mathfrak{b}_{i, j}$ repeats an indeterminate number of times. The 3 -regions $\mathfrak{s}_{3}, \mathfrak{s}_{6}, \mathfrak{s}_{6}^{\prime}$ and $\mathfrak{d}_{3}$ are as in Fig. 21 .

now to all regions depicted in either Fig. 19 or Fig. 21, and we further stipulate

$$
z \cdot\left(s_{0}+\cdots+s_{9}+s_{1}^{\prime}+\cdots+s_{8}^{\prime}+d_{1}+\cdots+d_{4}\right)=0 .
$$

Note that $d_{5}$ does not appear in this constraint; thus, $z^{\circ}$ may intersect the arc $\chi_{3}$. Again, these additional constraints are evidently satisfiable.

Now suppose we are given any collection of regions (not necessarily polygonal) satisfying (38)- 45). And let the arcs $\gamma_{0}, \ldots, \gamma_{9}, \gamma_{8}^{\prime}, \ldots, \gamma_{1}^{\prime}$ and $\chi_{1}, \chi_{2}, \chi_{3}$ be as defined above. It will be convenient in this stage to rename $\gamma_{6}$ and $\gamma_{6}^{\prime}$ as $\lambda_{0}$ and $\mu_{0}$, respectively. Thus, $\lambda_{0}$ forms the bottom edge of the lower window, and $\mu_{0}$ the top edge of the upper window. Likewise, we rename $\gamma_{3}$ as $\alpha_{0}$, forming 
part of the left-hand side of the lower window. Let $\tilde{q}_{1,1}$ be any point of $\alpha_{0}, p^{*}$ any point of $\lambda_{0}$, and $q^{*}$ any point of $\mu_{0}$; see Fig. 22. By (42), then, $\tilde{q}_{1,1} \in \dot{a}_{0,3}$, $p^{*} \in \ddot{a}$, and $q^{*} \in \ddot{b}$. Certainly, the constraint 45$)$ ensures that $\tilde{q}_{1,1} \in(-\dot{z})$. By Lemma 16 and (43), we may draw an arc $\tilde{\beta}_{1}$ from $\tilde{q}_{1,1}$ to $q^{*}$, with successive segments $\beta_{1,1}, \beta_{1,2}, \ldots, \beta_{1,5}, \tilde{\beta}_{1,6}$ lying in the respective regions

$$
a_{0,3}+b_{1,1}, \quad b_{1,2}, \ldots, \quad b_{1,5}, \quad b_{1,6}+b
$$

further, we can guarantee that $\beta_{1,2}$ contains a point $\tilde{p}_{1,1} \in \dot{b}_{1,3}$. Denote the last point of $\beta_{1,5}$ by $q_{1,2}$. Also, let $q_{1,1}$ be the last point of $\tilde{\beta}_{1}$ lying on $\alpha_{0}$, and $q_{1,3}$ the first point of $\tilde{\beta}_{1}$ lying on $\mu_{0}$. Finally, let $\beta_{1}$ be the segment of $\tilde{\beta}_{1}$ between $q_{1,1}$ and $q_{1,2}$; and let $\mu_{1}$ be the segment of $\tilde{\beta}_{1}$ from $q_{1,2}$ to $q_{1,3}$ followed by the final segment of $\mu_{0}$ from $q_{1,3}$; see Fig. 22. By repeatedly using the constraints in (41), it is easy to see that $\beta_{1}$ and the initial segment of $\mu_{1}$ up to $q_{1,3}$ together form a chord of $\Gamma$. Adding the constraint

$$
c\left(b_{1,5}+d_{3}\right),
$$

and taking into account the constraints in (41) ensures that this chord divides the residual domain of $\Gamma$ containing $\chi_{2}$ into the regular closed sets $S_{1}$ and $S_{1}^{\prime}$, as shown in Fig. 22. The wiggly lines indicate that we do not care about the exact positions of $\tilde{q}_{1,1}$ or $q^{*}$; otherwise, Fig. 22 is again completely general. Note that $\mu_{1}$ lies entirely in $b_{1,6}+b$, and hence certainly in the region

$$
b^{*}=b_{0,6}+b_{1,6}+b_{2,6}+b .
$$

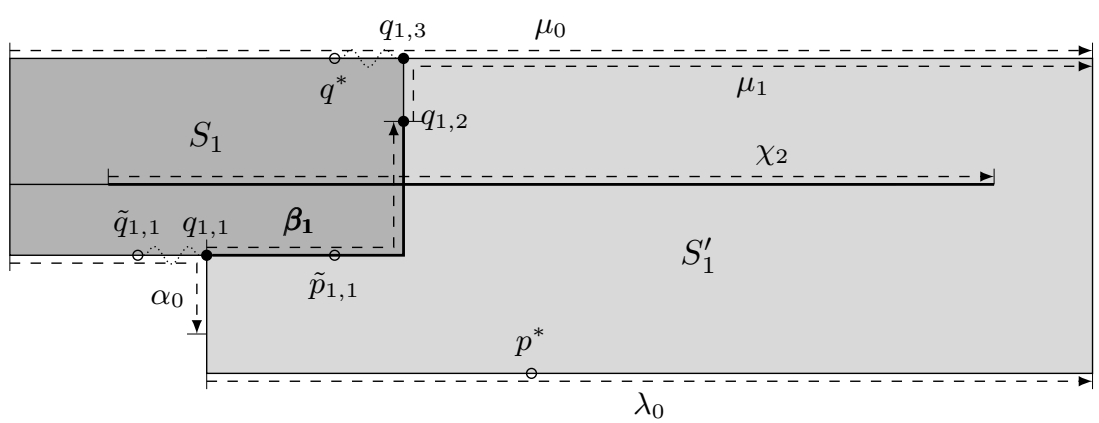

Figure 22: The $\operatorname{arc} \beta_{1}$.

Recall that $\tilde{p}_{1,1} \in \dot{b}_{1,3}$ and $p^{*} \in \ddot{a}$. By Lemma 16 and 44, we may draw an arc $\tilde{\alpha}_{1}$ from $\tilde{p}_{1,1}$ to $p^{*}$, with successive segments $\tilde{\alpha}_{1,1}, \alpha_{1,2}, \ldots, \alpha_{1,5}, \tilde{\alpha}_{1,6}$ lying in the respective regions

$$
b_{1,3}+a_{1,1}, \quad a_{1,2}, \quad \ldots, \quad a_{1,5}, \quad a_{1,6}+a
$$


further, we can guarantee that the segment $\alpha_{1,2}$ contains a point $\tilde{q}_{2,1} \in \dot{a}_{1,3}$. (Thus: $\alpha_{1,2}$ lies in $a_{1,2}$, but nevertheless contains at least one point lying in $\dot{a}_{1,3}$.) Denote the last point of $\alpha_{1,5}$ by $p_{1,2}$. Also, let $p_{1,1} \in b_{1,3}$ be the last point of $\tilde{\alpha}_{1}$ lying on $\beta_{1}$, and $p_{1,3}$ the first point of $\tilde{\alpha}_{1}$ lying on $\lambda_{0}$. From (41), and these points must be arranged as shown in Fig. 23. In particular, the segment of $\tilde{\alpha}_{1}$ between $p_{1,1}$ and $p_{1,3}$ is a chord in $S_{1}^{\prime}$ and divides it into regions $R_{1}$ and $R_{1}^{\prime}$. Let $\alpha_{1}$ be the segment of $\tilde{\alpha}_{1}$ between $p_{1,1}$ and $p_{1,2}$. Noting that 41] entails

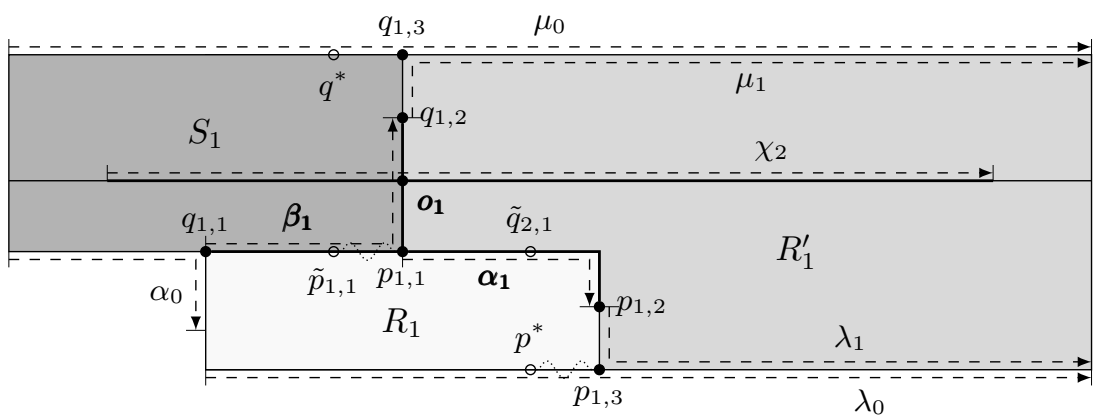

Figure 23: The $\operatorname{arcs} \beta_{1}$ and $\alpha_{1}$.

$$
\neg C\left(a_{1,1}+\cdots+a_{1,6}, \quad s_{0}+s_{9}+d_{0}+\cdots+d_{5}\right),
$$

we can be sure that $\alpha_{1}$ lies entirely in the 'lower' window, whence $\beta_{1}$ crosses the central chord, $\chi_{2}$ at least once. Let $o_{1}$ be the first such point (measured along $\chi_{2}$ from left to right). Finally, let $\lambda_{1}$ be the segment of $\tilde{\alpha}_{1}$ between $p_{1,2}$ and $p_{1,3}$, followed by the final segment of $\lambda_{0}$ from $p_{1,3}$. Note that $\lambda_{1}$ lies entirely in $a_{1,6}+a$, and hence certainly in the region

$$
a^{*}=a_{0,6}+a_{1,6}+a_{2,6}+a .
$$

The region $S_{1}$ may now be forgotten.

By construction, the point $\tilde{q}_{2,1}$ lies in some component of $\dot{a}_{1,3}$, and, from the presence of the 'switching' variable $\dot{z}$ in (44), that component is either included in $\dot{z}$ or included in $-\dot{z}$. Suppose the latter. Then we can repeat the above construction to obtain an $\operatorname{arc} \tilde{\beta}_{2}$ from $\tilde{q}_{2,1}$ to $q^{*}$, with successive segments $\tilde{\beta}_{2,1}$, $\beta_{2,2}, \ldots, \beta_{2,5}, \tilde{\beta}_{2,6}$ lying in the respective regions $a_{1,3}+b_{2,1}, b_{2,2}, \ldots, b_{2,5}$, $b_{2,6}+b$; further, we can guarantee that $\beta_{2,2}$ contains a point $\tilde{p}_{2,1} \in \dot{b}_{2,3}$. Denote the last point of $\beta_{2,5}$ by $q_{2,2}$. Also, let $q_{2,1}$ be the last point of $\tilde{\beta}_{2}$ lying on $\alpha_{1}$, and $q_{2,3}$ the first point of $\tilde{\beta}_{2}$ lying on $\mu_{1}$. Again, we let $\beta_{2}$ be the segment of $\tilde{\beta}_{2}$ between $q_{2,1}$ and $q_{2,2}$; and we let $\mu_{2}$ be the segment of $\tilde{\beta}_{2}$ from $q_{2,1}$ to $q_{2,3}$, followed by the final segment of $\mu_{1}$ from $q_{2,3}$. Note that $\mu_{2}$ lies in the set $b^{*}$. It is easy to see that the segment of $\tilde{\beta}_{2}$ from $q_{2,1}$ to $q_{2,3}$ is a cross-cut in $R_{1}^{\prime}$ dividing it into regions $S_{2}$ and $S_{2}^{\prime}$, as shown in Fig. 24 . Indeed, $\beta_{2}=\tilde{\beta}_{2}\left[q_{2,1}, q_{2,2}\right]$ cannot 
enter the interior of the region $R_{1}$, for, by construction, it can have only one point of contact with $\alpha_{1}$, and the constraints (41) ensure that it cannot intersect any other part of $\delta R_{1}$. Since $q^{*} \in \ddot{a}$ is guaranteed to lie outside $R_{1}$, we evidently have that $\beta_{2} \subseteq-R_{1}$. By the constraints (41), $\beta_{2}$ lies in the interior of $R_{1}^{\prime}$ except for its first point, which lies on the boundary of $R_{1}^{\prime}$; hence the reversal of $\beta_{2}$ is an end-cut in $R_{1}^{\prime}$. Similarly, $\tilde{\beta}_{2}\left[q_{2,2}, q_{2,3}\right]$ is an end-cut in $R_{1}^{\prime}$ as well, and thus $\tilde{\beta}_{2}\left[q_{2,1}, q_{2,3}\right]$ is a cross-cut in $R_{1}^{\prime}$. This observation having been made, $R_{1}$ may now be forgotten.

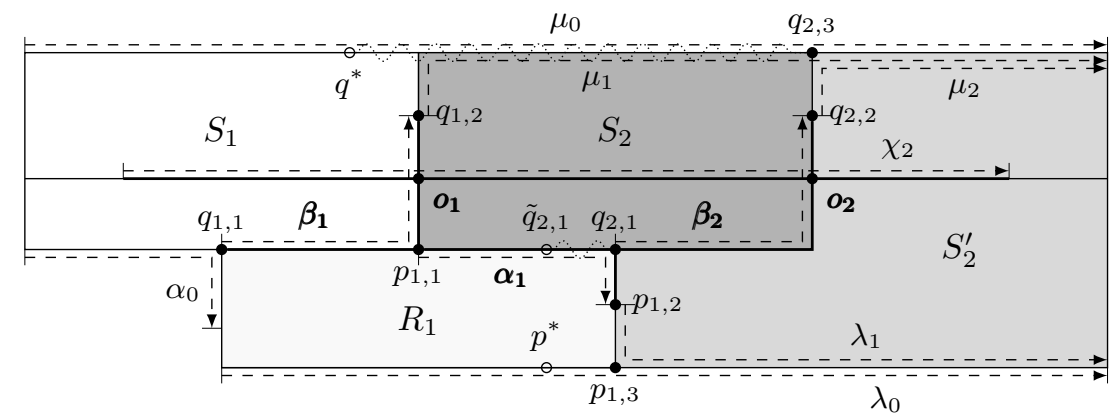

Figure 24: The $\operatorname{arc} \beta_{2}$.

Symmetrically, we construct the arc $\tilde{\alpha}_{2}$ in $b_{2,3}+a_{2,1}+\cdots+a_{2,6}+a$, and points $p_{2,1}, p_{2,2}, p_{2,3}$, together with the $\operatorname{arcs} \alpha_{2}$ and $\lambda_{2}$. Again, we know from (41) that $\alpha_{2}$ lies entirely in the 'lower' window, whence $\beta_{2}$ must cross the central chord, $\chi_{2}$, at least once. Let $o_{2}$ be the first such point (measured along $\chi_{2}$ from left to right); see Fig. 24

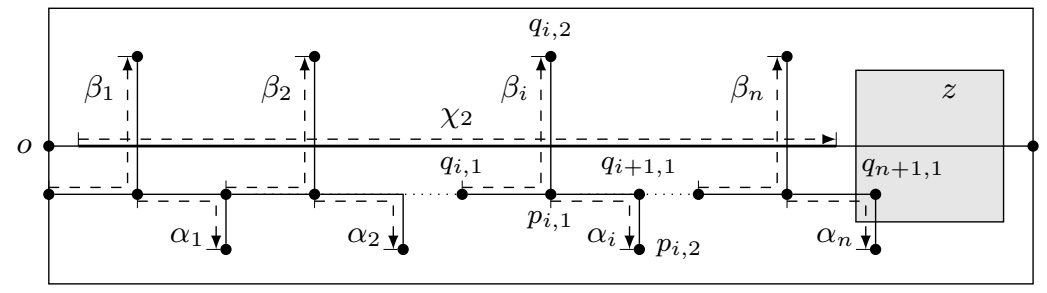

Figure 25: The sequence of pairs of $\operatorname{arcs}\left(\beta_{i}, \alpha_{i}\right)$.

This process continues, generating $\operatorname{arcs} \beta_{i} \subseteq a_{\lfloor i-1\rfloor, 3}+b_{\lfloor i\rfloor, 1}+\cdots+b_{\lfloor i\rfloor, 5}$ and $\alpha_{i} \subseteq b_{\lfloor i\rfloor, 3}+a_{\lfloor i\rfloor, 1}+\cdots+a_{\lfloor i\rfloor, 5}$, as long as $\alpha_{i}$ contains a point $\tilde{q}_{i+1,1} \in(-\dot{z})$. That we eventually reach a value $i=n$ for which no such point exists follows from (41). For the conjuncts $\neg C\left(b_{i, j}, d_{k}\right)$, for $j \neq 5$, together entail $o_{i} \in b_{\lfloor i\rfloor, 5}$, for every $i$ such that $\beta_{i}$ is defined; and these points cycle on $\chi_{2}$ through the regions $b_{0,5}, b_{1,5}$ and $b_{2,5}$. If there were infinitely many $\beta_{i}$, the $o_{i}$ would have an accumulation point, lying in all three regions, contradicting, say, $\neg C\left(b_{0,5}, b_{1,5}\right)$. 
The resulting sequence of arcs and points is shown, schematically, in Fig. 25. It follows that the final arc $\alpha_{n}$ contains a point $q_{n+1,1} \in \dot{z}$.

We finish this stage in the construction by 're-packaging' the pairs of arcs $\left(\beta_{i}, \alpha_{i}\right)$. Specifically, for all $1 \leq i \leq n$, let $\zeta_{i}$ be the initial segment of $\beta_{i}$ up to the point $p_{i, 1}$ followed by the initial segment of $\alpha_{i}$ up to the point $q_{i+1,1}$; let $\eta_{i}$ be the final segment of $\beta_{i}$ from the point $p_{i, 1}$; and let $\kappa_{i}$ be the final segment of $\alpha_{i}$ from the point $q_{i+1,1}$ :

$$
\zeta_{i}=\beta_{i}\left[q_{i, 1}, p_{i, 1}\right] \alpha_{i}\left[p_{i, 1}, q_{i+1,1}\right], \quad \eta_{i}=\beta_{i}\left[p_{i, 1}, q_{i, 2}\right], \quad \kappa_{i}=\alpha_{i}\left[q_{i+1,1}, p_{i, 2}\right]
$$

(see Fig. 26). Defining, for $0 \leq i<3$,

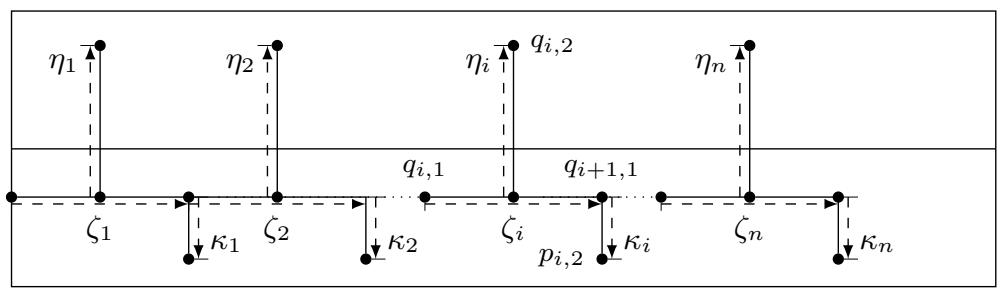

Figure 26: The sequence of triples of $\operatorname{arcs}\left(\zeta_{i}, \eta_{i}, \kappa_{i}\right)$ formed by 're-packaging' $\left(\beta_{i}, \alpha_{i}\right)$ from Fig. 25.

$$
\begin{aligned}
r_{i} & =a_{\lfloor i-1\rfloor, 3}+b_{i, 1}+\cdots+b_{i, 4}+a_{i, 1}+\cdots+a_{i, 4}, \\
b_{i} & =b_{i, 2}+\cdots+b_{i, 5}, \\
a_{i} & =a_{i, 2}+\cdots+a_{i, 5},
\end{aligned}
$$

the constraints 41 guarantee that, for $1 \leq i \leq n$,

$$
\zeta_{i} \subseteq r_{\lfloor i\rfloor}, \quad \eta_{i} \subseteq b_{\lfloor i\rfloor} \quad \text { and } \quad \kappa_{i} \subseteq a_{\lfloor i\rfloor} .
$$

Observe that the $\operatorname{arcs} \zeta_{i}$ are located entirely in the 'lower window,' and that each arc $\eta_{i}$ connects $\zeta_{i}$ to some point $q_{i, 2}$, which in turn is connected to $q^{*} \in \lambda_{0}$ by an $\operatorname{arc}$ in $b^{*}$.

Stage 3. We now repeat Stage 2 symmetrically, with the 'upper' and 'lower' windows exchanged. Let $\mathfrak{a}_{i, j}^{\prime}, \mathfrak{b}_{i, j}^{\prime}$ be 3 -region variables (with indices in the same ranges as for $\left.\mathfrak{a}_{i, j}, \mathfrak{b}_{i, j}\right)$. Let $\mathfrak{a}^{\prime}=\mathfrak{b}, \mathfrak{b}^{\prime}=\mathfrak{a}$. The formulas

$$
\begin{aligned}
& \left(s_{3}^{\prime} \leq \dot{a}_{0,3}^{\prime}\right), \\
& \bigwedge_{i=0}^{2} \operatorname{stack}_{\dot{z}}\left(\mathfrak{a}_{\lfloor i-1\rfloor, 3}^{\prime}, \mathfrak{b}_{i, 1}^{\prime}, \ldots, \mathfrak{b}_{i, 6}^{\prime}, \mathfrak{b}^{\prime}\right), \\
& \bigwedge_{i=0}^{2} \operatorname{stack}\left(\mathfrak{b}_{i, 3}^{\prime}, \mathfrak{a}_{i, 1}^{\prime}, \ldots, \mathfrak{a}_{i, 6}^{\prime}, \mathfrak{a}^{\prime}\right),
\end{aligned}
$$




$$
c\left(b_{1,5}^{\prime}+d_{3}\right)
$$

then establish sequences of $n^{\prime}$ triples of $\operatorname{arcs}\left(\zeta_{i}^{\prime}, \eta_{i}^{\prime}, \kappa_{i}^{\prime}\right)$ satisfying

$$
\zeta_{i}^{\prime} \subseteq r_{\lfloor i\rfloor}^{\prime}, \quad \eta_{i}^{\prime} \subseteq b_{\lfloor i\rfloor}^{\prime} \quad \text { and } \quad \kappa_{i} \subseteq a_{\lfloor i\rfloor}^{\prime},
$$

for $1 \leq i \leq n^{\prime}$, where the $r_{i}^{\prime}, b_{i}^{\prime}$ and $a_{i}^{\prime}$ are defined as in 49 - 51) but with the primed variables. The $\operatorname{arcs} \zeta_{i}^{\prime}$ are located entirely in the 'upper window', and each arc $\eta_{i}^{\prime}$ connects $\zeta_{i}^{\prime}$ to a point $q_{i, 2}^{\prime}$, which in turn is connected to a point $q^{* \prime}$ by an arc in the region $b^{* \prime}=b_{0,6}^{\prime}+b_{1,6}^{\prime}+b_{2,6}^{\prime}+b^{\prime}$.

Stage 4. Our next task is to write constraints to ensure that $n=n^{\prime}$, and that, furthermore, each $\eta_{i}$ (also each $\eta_{i}^{\prime}$ ) connects $\zeta_{i}$ to $\zeta_{i}^{\prime}$, for $1 \leq i \leq n$. From (45), the only arc depicted in Fig. 20 that $z^{\circ}$ may intersect is $\chi_{3}$. Recalling that $\zeta_{n}$ and $\zeta_{n^{\prime}}^{\prime}$ contain points $q_{n+1,1}$ and $q_{n^{\prime}+1,1}^{\prime}$, respectively, both lying in $\dot{z} \subseteq z^{\circ}$, the constraint

$$
c(\dot{z})
$$

ensures that $q_{n+1,1}$ and $q_{n^{\prime}+1,1}^{\prime}$ may be joined by an arc, say $\zeta^{*}$, lying in $z^{\circ}$, and also lying entirely in the upper and lower windows, crossing the chord $\chi_{1} \chi_{2} \chi_{3}$ only in $\chi_{3}$. Without loss of generality, we may assume that $\zeta^{*}$ contacts each of $\zeta_{n}$ and $\zeta_{n^{\prime}}^{\prime}$ in just one point. Bearing in mind that the formulas (41) force $\eta_{n} \subseteq b_{0}+b_{1}+b_{2}$ and $\eta_{n^{\prime}}^{\prime} \subseteq b_{0}^{\prime}+b_{1}^{\prime}+b_{2}^{\prime}$ to cross the chord $\chi_{1} \chi_{2} \chi_{3}$ in its central section, $\chi_{2}$, and bearing in mind (45), we see that the following constraint ensures that $\zeta^{*}$ is as shown in Fig. 27.

$$
z \cdot\left(b^{*}+b_{0}+b_{1}+b_{2}+b^{* \prime}+b_{0}^{\prime}+b_{1}^{\prime}+b_{2}^{\prime}\right)=0 .
$$

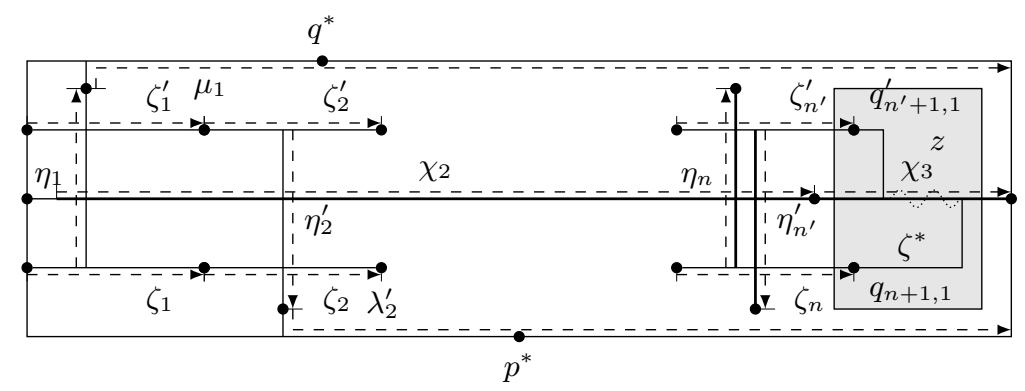

Figure 27: The $\operatorname{arc} \zeta^{*}$.

Now consider the arc $\eta_{1}$. Recalling that $\eta_{1}$ crosses $\chi_{2}$ and connects $\zeta_{1}$ to some point $q_{1,2}$, which in turn is connected to the point $q^{*}$ by an $\operatorname{arc}$ in $b^{*}$, we see by inspection of Fig. 27 that 53 together with

$$
\bigwedge_{i=0}^{2} \neg C\left(r_{i}^{\prime}, b^{*}\right)
$$


forces $\eta_{1}$ to cross one of the $\operatorname{arcs} \zeta_{j^{\prime}}^{\prime} \subseteq r_{\left\lfloor j^{\prime}\right\rfloor}^{\prime}$, for $1 \leq j^{\prime} \leq n^{\prime}$; and the constraints

$$
\bigwedge_{i=0}^{2} \neg C\left(r_{i}^{\prime}, \quad b_{\lfloor i-1\rfloor}+b_{\lfloor i+1\rfloor}\right)
$$

ensure that $j^{\prime} \equiv 1$ modulo 3 . Now suppose $j^{\prime} \geq 4$. We write the constraints

$$
\begin{aligned}
& \bigwedge_{i=0}^{2} \neg C\left(b_{i}^{\prime}, \quad r_{\lfloor i-1\rfloor}+r_{\lfloor i+1\rfloor}\right), \\
& \bigwedge_{i=0}^{2} \neg C\left(b_{i}^{\prime}, \quad b_{\lfloor i-1\rfloor}+b_{\lfloor i+1\rfloor}\right) .
\end{aligned}
$$

The arc $\eta_{2}^{\prime}$ must connect $\zeta_{2}^{\prime}$ to the point $q_{2,2}^{\prime}$, which in turn is connected to the point $p^{*}$ on the bottom edge of the lower window by an arc in $b^{* \prime}$, which is now impossible without $\eta_{2}^{\prime} \subseteq b_{2}^{\prime}$ crossing either $\zeta_{1} \subseteq r_{1}$ or $\eta_{1} \subseteq b_{1}$-both forbidden by (55]- (56). Thus, $\eta_{1}$ intersects $\zeta_{j}^{\prime}$ if and only if $j=1$. Symmetrically, $\eta_{1}^{\prime}$ intersects $\zeta_{j}$ if and only if $j=1$. And the reasoning can now be repeated for $\eta_{2}, \eta_{2}^{\prime}, \eta_{3}, \eta_{3}^{\prime}, \ldots$, leading to the $1-1$ correspondence depicted in Fig. 28. In particular, we are guaranteed that $n=n^{\prime}$.

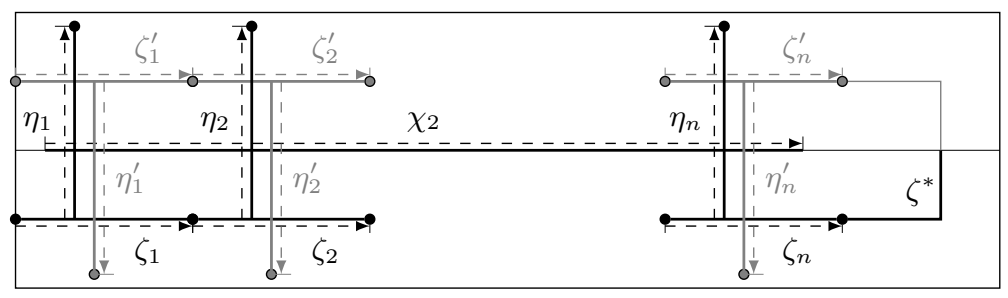

Figure 28: The 1-1 correspondence between the $\zeta_{i}$ and the $\zeta_{i}^{\prime}$ established by the $\eta_{i}$ and the $\eta_{i}^{\prime}$.

Stage 5. Recall the given PCP-instance, $\mathrm{W}=\left(\mathrm{w}, \mathrm{w}^{\prime}\right)$ over alphabets $T$ and $U$. In the sequel, we use the standard imagery of 'tiles', where each tile $t \in T$ has an 'upper string', $w^{\prime}(t) \in U^{*}$ and a 'lower string', $w(t) \in U^{*}$. Thus, the problem is to determine whether there is some non-empty sequence of tiles such that the concatenated upper and lower strings both spell out the same string in $U^{*}$. We shall label the $\operatorname{arcs} \zeta_{1}, \ldots, \zeta_{n}$ so as to define a string $\tau \in T^{*}($ with $|\tau|=m \leq n)$; likewise we shall label the $\operatorname{arcs} \zeta_{1}^{\prime}, \ldots, \zeta_{n}^{\prime}$ so as to define another string $\tau^{\prime} \in T^{*}$ (with $\left|\tau^{\prime}\right|=m^{\prime} \leq n$ ). Then the arcs $\eta_{1}, \ldots, \eta_{n}$ will be labelled with the regions in $\vec{u}$, so to define a string $v \in U^{*}$, with $|v|=n$. We shall then add conjuncts to $\psi_{\mathrm{w}}$ ensuring $\mathrm{w}(\tau)=\mathrm{w}^{\prime}\left(\tau^{\prime}\right)=v$ and $\tau=\tau^{\prime}$, which will guarantee that $\mathrm{W}$ is positive.

For all $1 \leq h \leq|T|, 1 \leq \ell \leq\left|\mathrm{w}\left(t_{h}\right)\right|$ and $0 \leq i<3$, let $p_{h, \ell}$ be a fresh variable, and let these variables be ordered in some way as the tuple $\vec{p}$. As in 
the proof of Theorem 8 , we think of $p_{h, \ell}$ as standing for the $\ell$ th position in the string $\mathrm{w}\left(t_{h}\right)$, where $t_{h} \in T$. We use $\vec{p}$ to label the components of $r_{i}, 0 \leq i<3$, but since the $r_{i}$ are not pairwise disjoint, we require a copy of the variables $\vec{p}$ for each $i$. Hence, for all $1 \leq h \leq|T|, 1 \leq \ell \leq\left|\mathrm{w}\left(t_{h}\right)\right|$ and $0 \leq i<3$, let $p_{h, \ell}^{i}$ be a fresh variable, and let $\vec{p}_{i}$ be an ordering of the variables with superscript $i$. Consider the constraints

$$
\bigwedge_{i=0}^{2}\left(\left(r_{i}=\sum_{h=1}^{|T|} \sum_{\ell=1}^{\left|\mathrm{w}\left(t_{h}\right)\right|} p_{h, \ell}^{i}\right) \wedge \operatorname{colour}\left(r_{i} ; \vec{p}_{i}\right)\right) \wedge \bigwedge_{h=1}^{|T|} \bigwedge_{\ell=1}^{\left|\mathrm{w}\left(t_{h}\right)\right|}\left(p_{h, \ell}=\sum_{i=0}^{2} p_{h, l}^{i}\right) .
$$

The first conjunct ensures that each arc $\beta_{i, 3} \subseteq b_{\lfloor i\rfloor, 3}(1 \leq i \leq n)$ is included in exactly one of the regions $\vec{p}_{\lfloor i\rfloor}$ and is disjoint from the rest of the regions in $\vec{p}_{\lfloor i\rfloor}$ and all the regions in $\vec{p}_{\lfloor i-1\rfloor}$ and $\vec{p}_{\lfloor i+1\rfloor}$; the second conjunct then ensures that $\zeta_{i}$ is contained in exactly one of the regions $\vec{p}$, and that $\beta_{i, 3}$ is disjoint from the rest of the regions in $\vec{p}$. Note that the $\vec{p}$ do not actually form a partition, because they cannot be made disjoint; nevertheless, we can think of the $\vec{p}$ as 'labels' for $\operatorname{arcs} \zeta_{i}$. The regions in $\vec{p}_{0}, \vec{p}_{1}$ and $\vec{p}_{2}$ can now be forgotten.

Next, we organize the $\operatorname{arcs} \zeta_{i}$ into (contiguous) blocks, $E_{1}, \ldots, E_{m}$ such that, in the $j$ th block, $E_{j}$, the sequence of labels reads $p_{h, 1}, \ldots, p_{h,\left|w\left(t_{h}\right)\right|}$, for some fixed $1 \leq h \leq|T|$. This amounts to insisting that: $(i)$ the very first arc, $\zeta_{1}$, must be labelled with $p_{h, 1}$ for some $h$; (ii) if $\zeta_{i}(i<n)$ is labelled with $p_{h, \ell}$, where $\ell<\left|\mathrm{w}\left(t_{h}\right)\right|$, then the next arc, namely $\zeta_{i+1}$, must be labelled with the next position in $\mathrm{w}\left(t_{h}\right)$, namely $p_{h, \ell+1} ;(i i i)$ if $\zeta_{i}(i<n)$ is labelled with the final position of $\mathrm{w}\left(t_{h}\right)$, then the next arc must be labelled with the initial position of some possibly different word $\mathrm{w}\left(t_{h^{\prime}}\right)$; and $(i v) \zeta_{n}$ must be labelled with the final position of some word $\mathrm{w}\left(t_{h}\right)$. To do this we simply write:

$$
\begin{aligned}
& |T|\left|w\left(t_{h}\right)\right| \\
& \bigwedge_{h=1} \bigwedge_{\ell=2} \neg C\left(p_{h, \ell}, s_{3}\right), \\
& \bigwedge_{i=0}^{2} \bigwedge_{h=1}^{|T|} \bigwedge_{\ell=1}^{\left|\mathrm{w}\left(t_{h}\right)-1\right|} \neg C\left(r_{i} \cdot p_{h, \ell}, r_{\lfloor i+1\rfloor} \cdot\left(-r_{i}\right) \cdot\left(\sum_{\ell^{\prime} \neq \ell+1} p_{h, \ell^{\prime}}+\sum_{h^{\prime} \neq h} \sum_{\ell^{\prime}=1}^{\left|\mathrm{w}\left(t_{h^{\prime}}\right)\right|} p_{h^{\prime}, \ell^{\prime}}\right)\right), \\
& \bigwedge_{i=0}^{2} \bigwedge_{h=1}^{|T|} \bigwedge_{h^{\prime}=1}^{|T|} \bigwedge_{\ell=2}^{\left|\mathrm{w}\left(t_{h^{\prime}}\right)\right|} \neg C\left(r_{i} \cdot p_{h,\left|\mathrm{w}\left(t_{h}\right)\right|}, \quad r_{\lfloor i+1\rfloor} \cdot\left(-r_{i}\right) \cdot p_{h^{\prime}, \ell}\right) \\
& |T|\left|w\left(t_{h}\right)\right|-1 \\
& \bigwedge_{h=1} \bigwedge_{\ell=1} \neg C\left(p_{h, \ell}, z\right) .
\end{aligned}
$$

Supposing the arcs of $j$ th block $E_{j}$ to have labels reading $p_{h, 1}, \ldots, p_{h,\left|\mathrm{w}\left(t_{h}\right)\right|}$ (for some fixed $h$ ), then, we write $h_{j}$ to denote the common subscript $h$. The sequence of indices $h_{1}, \ldots, h_{m}$ corresponding to the successive blocks thus defines a word $\tau=t_{h_{1}} \cdots t_{h_{m}} \in T^{*}$.

Using corresponding formulas, we label the $\operatorname{arcs} \zeta_{i}^{\prime}(1 \leq i \leq n)$ with the tuple $\vec{p}^{\prime}$ of variables $p_{h, \ell}^{\prime}$, for $1 \leq h \leq|T|$ and $1 \leq \ell \leq\left|\mathbf{w}^{\prime}\left(t_{h}\right)\right|$, so that, in 
any satisfying assignment over $\mathrm{RC}\left(\mathbb{R}^{2}\right)$, every arc $\zeta_{i}^{\prime}$ is labeled with exactly one of the regions $\vec{p}^{\prime}$ and $\beta_{i, 3}^{\prime} \subseteq b_{i, 3}$ is disjoint from the rest of the regions in $\vec{p}^{\prime}$. Further, we can ensure that these labels are organized into (say) $m^{\prime}$ contiguous blocks, $E_{1}^{\prime}, \ldots, E_{m^{\prime}}^{\prime}$ such that in the $j$ th block, $E_{j}^{\prime}$, the sequence of labels reads $p_{h, 1}^{\prime}, \ldots, p_{h,\left|\mathrm{w}^{\prime}\left(t_{h}\right)\right|}^{\prime}$, for some fixed $h$. Again, writing $h_{j}^{\prime}$ for the common value of $h$, the sequence of indices $h_{1}^{\prime}, \ldots, h_{m^{\prime}}^{\prime}$ corresponding to the successive blocks defines a word $\tau^{\prime}=t_{h_{1}^{\prime}} \cdots t_{h_{m^{\prime}}^{\prime}} \in T^{*}$.

Now, the constraints

$$
\operatorname{part}(\vec{u}) \wedge \bigwedge_{i=0}^{2} \operatorname{colour}\left(b_{i} ; \vec{u}\right)
$$

ensure that, in any satisfying assignment over $\mathrm{RC}\left(\mathbb{R}^{2}\right)$, every arc $\eta_{i} \subseteq b_{\lfloor i\rfloor}$, for $1 \leq i \leq n$, is included in ('labelled with') exactly one of the regions in $\vec{u}$, so that the sequence of arcs $\eta_{1}, \ldots, \eta_{n}$ defines a string $v \in U^{*}$, with $|v|=n$.

Securing $\mathrm{w}(\tau)=\mathrm{w}^{\prime}\left(\tau^{\prime}\right)=v$ is easy. The constraints

$$
\bigwedge_{h=1}^{|T|\left|\mathrm{w}\left(t_{h}\right)\right|} \bigwedge_{\ell=1} \bigwedge_{\begin{array}{c}
u_{i} \text { is not the } \ell \text { th } \\
\text { letter of } \mathrm{w}\left(t_{h}\right)
\end{array}} \neg C\left(u_{i}, p_{h, \ell}\right)
$$

ensure that, since $\eta_{i}$ intersects $\zeta_{i}$, for all $1 \leq i \leq n$, the string $v \in U^{*}$ defined by the arcs $\eta_{i}$ must be identical to the string $\mathrm{w}\left(t_{h_{1}}\right) \cdots \mathrm{w}\left(t_{h_{m}}\right)$. But this is just to say that $v=\mathrm{w}(\tau)$. The equation $v=\mathrm{w}^{\prime}\left(\tau^{\prime}\right)$ is obtained similarly.

Stage 6. In the foregoing stages, we assembled conjuncts of $\psi_{\mathrm{w}}$ in such a way that, given any satisfying assignment for $\psi_{\mathrm{W}}$, we can construct sequences of labelled arcs defining words $v \in U^{*}$ and $\tau, \tau^{\prime} \in T^{*}$ with $\mathrm{w}(\tau)=\mathrm{w}^{\prime}\left(\tau^{\prime}\right)=v$, as described above. In this stage, we add more conjuncts to $\psi_{\mathrm{W}}$ to enforce the equation $\tau=\tau^{\prime}$. This shows that, if $\psi_{\mathrm{W}}$ is satisfiable over $\operatorname{RC}\left(\mathbb{R}^{2}\right)$, then $\mathrm{W}$ is positive.

In particular, it remains to show that $m=m^{\prime}$ and that $h_{j}=h_{j}^{\prime}$, for all $1 \leq j \leq m$. To do so, we re-use the techniques encountered in Stage 4. We first introduce a new pair of variables, $f_{0}, f_{1}$, which we refer to as 'block colours', and with which we label the $\operatorname{arcs} \zeta_{i}$. Again, since the regions $r_{i}$ overlap, we additionally require regions $f_{0}^{i}$ and $f_{1}^{i}$, for $0 \leq i<3$. Consider the constraints:

$$
\bigwedge_{i=0}^{2}\left(\left(r_{i}=f_{0}^{i}+f_{1}^{i}\right) \wedge \operatorname{colour}\left(r_{i} ; f_{0}^{i}, f_{1}^{i}\right)\right) \wedge \bigwedge_{k=0}^{1}\left(f_{k}=\sum_{i=0}^{2} f_{k}^{i}\right) .
$$

It is readily checked that each $\zeta_{i} \subseteq r_{\lfloor i\rfloor}$ is included in exactly one of the regions $f_{0}$ or $f_{1}$, and that $\beta_{i, 3}$ is disjoint from the other. (Again, however, $f_{0}, f_{1}$ do not form a partition, because they must overlap.) We force all arcs in each block $E_{j}$ to have a uniform block colour, and we force the block colours to alternate by writing:

$$
\bigwedge_{k=0}^{1} \bigwedge_{h=1}^{|T|} \bigwedge_{\ell=1}^{\left|w\left(t_{h}\right)\right|-1} \neg C\left(f_{k} \cdot p_{h, \ell}, \quad f_{1-k} \cdot p_{h, \ell+1}\right),
$$




$$
\bigwedge_{k=0}^{1} \bigwedge_{h=1}^{|T|} \bigwedge_{h^{\prime}=1}^{|T|} \bigwedge_{i=0}^{2} \neg C\left(f_{k} \cdot p_{h,\left|\mathrm{w}\left(t_{h}\right)\right|} \cdot r_{i}, \quad f_{k} \cdot p_{h^{\prime}, 1} \cdot r_{\lfloor i+1\rfloor} \cdot\left(-r_{i}\right)\right) .
$$

Thus, we may speak unambiguously of the colour $\left(f_{0}\right.$ or $\left.f_{1}\right)$ of a block: if $E_{1}$ is coloured $f_{0}$, then $E_{2}$ will be coloured $f_{1}, E_{3}$ coloured $f_{0}$, and so on. Using variables $f_{0}^{\prime}$ and $f_{1}^{\prime}$, we similarly establish a block structure $E_{1}^{\prime}, \ldots, E_{m^{\prime}}^{\prime}$ on the $\operatorname{arcs} \zeta_{i}^{\prime}$.

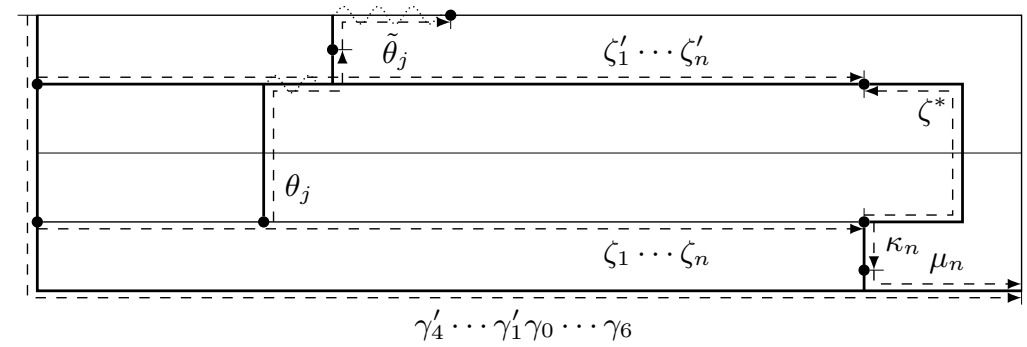

Figure 29: $\operatorname{Arc} \theta_{j} \tilde{\theta}_{j}$ intersecting $\zeta_{1}^{\prime} \cdots \zeta_{n}^{\prime}$.

Now we match up the blocks in a 1-1 fashion, just as we matched up the individual arcs in Stage 4. Let $\mathfrak{g}_{0}, \mathfrak{g}_{1}, \mathfrak{g}_{0}^{\prime}$ and $\mathfrak{g}_{1}^{\prime}$ be new 3-region variables. Recall that every arc $\zeta_{i}$ contains some point of $b_{\lfloor i\rfloor, 3}$ (for instance: $p_{i, 1}$ ) and every such point is unambiguously labeled by a region in $\vec{p}$ and a region in $\left(f_{0}, f_{1}\right)$. We wish to connect any such arc that starts a block $E_{j}$ (i.e., any $\zeta_{i}$ labelled by $p_{h, 1}$ for some $h$ ) to the top edge of the upper window, with the connecting arc depending on the block colour. We can do this using the constraints:

$$
\bigwedge_{k=0}^{1}\left(\left(f_{k} \cdot\left(b_{0,3}+b_{1,3}+b_{2,3}\right) \leq \dot{g}_{k}\right) \wedge \operatorname{stack}\left(\mathfrak{g}_{k}, \mathfrak{b}\right)\right)
$$

Specifically, the first (actually: every) $\operatorname{arc} \zeta_{i}$ in each block $E_{j}$, for $1 \leq j \leq m$, is connected by an $\operatorname{arc} \theta_{j} \tilde{\theta}_{j}$ to some point on the upper edge of the upper window, where $\theta_{j} \subseteq g_{k}$ and $\tilde{\theta}_{j} \subseteq b$. Using corresponding formulas, we ensure that the first arc in each block $E_{j}^{\prime}$, for $1 \leq j \leq m^{\prime}$, is connected by an arc $\theta_{j}^{\prime} \tilde{\theta}_{j}^{\prime}$ to some point on the bottom edge of the lower window, where $\theta_{j}^{\prime} \subseteq g_{k}^{\prime}$ and $\tilde{\theta}_{j}^{\prime} \subseteq b^{\prime}$.

Recall from Stage 3 that $q_{n+1,1}$ is connected by an arc $\kappa_{n} \subseteq a_{0}+a_{1}+a_{2}$ to $p_{n, 2}$, which is in turn connected to the lower edge of the lower window by an arc in lying in $a^{*}$. And recall from Stage 4 that $q_{n+1,1}$ is connected by $\zeta^{*} \subseteq z^{\circ}$ to $q_{n+1,1}^{\prime}$. Thus, we see from Fig. 29 that the non-contact constraints

$$
\neg C\left(g_{0}+g_{1}, s_{4}^{\prime}+\cdots+s_{1}^{\prime}+s_{0}+\cdots+s_{5}+a^{*}+a_{0}+a_{1}+a_{2}+z\right)
$$

ensure that each $\theta_{j} \tilde{\theta}_{j}(1 \leq j \leq m)$ intersects one of the $\zeta_{i}^{\prime}(1 \leq i \leq n)$. Indeed, since $\tilde{\theta}_{j} \subseteq b$ cannot intersect any $\zeta_{i}^{\prime}$, we know that all such points of intersection 
lie on $\theta_{j}$. Using a corresponding formula, we ensure that each $\theta_{j}^{\prime}\left(1 \leq j \leq m^{\prime}\right)$ intersects one of the $\zeta_{i}(1 \leq i \leq n)$.

We now write the constraints

$$
\bigwedge_{k=0}^{1}\left(\neg C\left(g_{k}, f_{1-k}^{\prime}\right) \wedge \neg C\left(g_{k}^{\prime}, f_{1-k}\right)\right) .
$$

Thus, any $\theta_{j}$ included in $g_{k}$ must join some $\operatorname{arc} \zeta_{i}$ in a block with colour $f_{k}$ to some arc $\zeta_{i^{\prime}}^{\prime}$ in a block with colour $f_{k}^{\prime}$; and symmetrically for the $\theta_{j}^{\prime}$. Adding

$$
\neg C\left(g_{0}+g_{0}^{\prime}, \quad g_{1}+g_{1}^{\prime}\right)
$$

then ensures, via reasoning similar to that employed in Stage 4 , that $\theta_{1}$ connects the block $E_{1}$ to the block $E_{1}^{\prime}, \theta_{2}$ connects $E_{2}$ to $E_{2}^{\prime}$, and so on; and similarly for the $\theta_{j}^{\prime}$ (as shown, schematically, in Fig. 30p. Thus, we have a $1-1$ correspondence between the two sets of blocks, whence $m=m^{\prime}$.

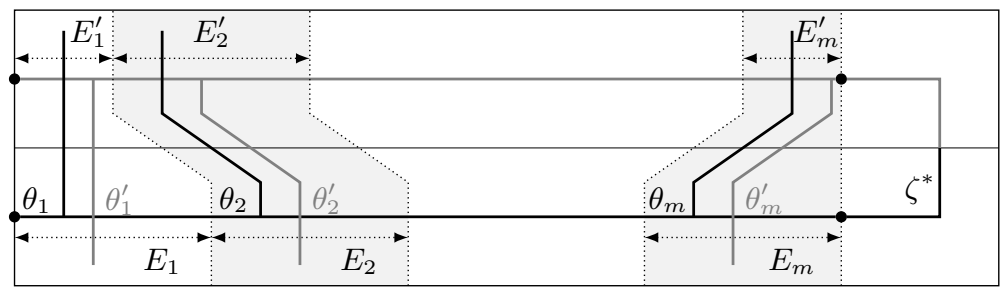

Figure 30: The 1-1 correspondence between the $E_{j}$ and the $E_{j}^{\prime}$ established by the $\theta_{j}$ and the $\theta_{j}^{\prime}$.

Finally, we regard elements of alphabet $T$ as fresh variables and order them to form tuple $\vec{t}$. These variables are used for labelling the components of $g_{0}$ and of $g_{1}$, and hence the $\operatorname{arcs} \theta_{1}, \ldots, \theta_{m}$ :

$$
\operatorname{part}(\vec{t}) \wedge \operatorname{colour}\left(g_{0} ; \vec{t}\right) \wedge \operatorname{colour}\left(g_{1} ; \vec{t}\right) .
$$

(Note that this time we can take the regions $\vec{t}$ to form a partition.) Adding the constraints

$$
\bigwedge_{k=0}^{1} \bigwedge_{h=1}^{|T|} \neg C\left(\sum_{\substack{1 \leq h^{\prime} \leq|T| \\ h^{\prime} \neq h}}\left(g_{k} \cdot t_{h^{\prime}}\right), \quad \sum_{\ell=1}^{\left|w\left(t_{h}\right)\right|} p_{h, \ell}+\sum_{\ell=1}^{\left|w^{\prime}\left(t_{h}\right)\right|} p_{h, \ell}^{\prime}\right)
$$

instantly ensures that the sequences of tile indices $h_{1}, \ldots, h_{m}$ and $h_{1}^{\prime}, \ldots, h_{m}^{\prime}$ are identical. In other words, $\tau=\tau^{\prime}$.

This completes the argument that, if $\psi_{\mathrm{W}}$ has a satisfying assignment over $\mathrm{RC}\left(\mathbb{R}^{2}\right)$, then $\mathrm{W}$ is a positive instance of the PCP. By extending the arrangement of Fig. 21 in the obvious way, we see that, if $W$ is a positive instance of the $\mathrm{PCP}$, then $\psi_{\mathrm{W}}$ has a satisfying assignment over $\mathrm{RCP}\left(\mathbb{R}^{2}\right)$, and hence (trivially) a satisfying assignment over $\mathrm{RC}\left(\mathbb{R}^{2}\right)$. 
The case $\mathcal{C} c^{\circ}$ is dealt with as for Corollary 6. we simply replace all occurrences of $c$ in $\psi_{\mathrm{W}}$ with $c^{\circ}$. Denoting the resulting $\mathcal{C} c^{\circ}$-formula by $\psi_{\mathrm{W}}^{\circ}$, we see that the following are equivalent: $(i) \mathrm{W}$ is positive; $(i i) \psi_{\mathrm{W}}^{\circ}$ is satisfiable over $\operatorname{RCP}\left(\mathbb{R}^{2}\right) ;($ iii $) \psi_{\mathrm{W}}^{\circ}$ is satisfiable over $\mathrm{RC}\left(\mathbb{R}^{2}\right)$. Thus,

Corollary 19. The problems $\operatorname{Sat}\left(\mathcal{C} c^{\circ}, \mathrm{RC}\left(\mathbb{R}^{2}\right)\right)$ and $\operatorname{Sat}\left(\mathcal{C} c^{\circ}, \mathrm{RCP}\left(\mathbb{R}^{2}\right)\right)$ are r.e.hard.

Employing the techniques of the proof of Theorem 7 , we show that

Theorem 20. The problems $\operatorname{Sat}\left(\mathcal{B} c, \mathrm{RC}\left(\mathbb{R}^{2}\right)\right)$ and $\operatorname{Sat}\left(\mathcal{B} c, \mathrm{RCP}\left(\mathbb{R}^{2}\right)\right)$ are r.e.hard.

Proof. Again, observe that all conjuncts of $\psi_{\mathrm{w}}$ featuring the predicate $C$ are negative (remember that there are additional such literals implicit in the use of 3-region variables, e.g., $(\dot{r} \ll r)$; but let us ignore these for the moment.) Recall the formula notC $(r, s)$ from the proof of Theorem 7 and consider the effect of replacing any literal $\neg C(r, s)$ in $\psi_{\mathrm{W}}$ by the corresponding instance of $\operatorname{notC}\left(r+r^{\prime}, s+s^{\prime}\right)$, where $r^{\prime}$ and $s^{\prime}$ are fresh variables; denote the resulting formula by $\psi$. It is easy to see that $\psi$ entails $\psi_{\mathrm{W}}$; hence if $\psi$ is satisfiable, then $\mathrm{W}$ is a positive instance of the PCP.

We next show that, if $\mathrm{W}$ is a positive instance of the $\mathrm{PCP}$, then $\psi$ is satisfiable over $\operatorname{RCP}\left(\mathbb{R}^{2}\right)$. For consider a tuple from $\operatorname{RCP}\left(\mathbb{R}^{2}\right)$ satisfying $\psi_{\mathrm{W}}$, and based on the arrangement of Fig. 21. Note that if $\mathfrak{r}$ and $\mathfrak{s}$ are 3-regions whose outer shells, $r$ and $s$ are not in contact (e.g., $\mathfrak{a}_{0,1}$ and $\mathfrak{a}_{0,3}$ ), then $r$ and $s$ have $(i)$ finitely many components, and (ii) connected complements. Hence, it is easy to find polygons $r^{\prime}$ and $s^{\prime}$ satisfying notC $\left(r+r^{\prime}, s+s^{\prime}\right)$. Fig. 31 represents the situation schematically. We may therefore assume that all such literals $\neg C(r, s)$ have been eliminated from $\psi_{\mathrm{w}}$.
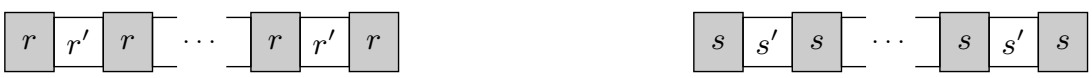

Figure 31: Disjoint connected regions $r+r^{\prime}$ and $s+s^{\prime}$ for regions $r$ and $s$ with finitely many components and connected complements.

We are not quite done, however. We must show that we can replace the implicit non-contact constraints $(\ddot{r} \ll \dot{r})$ and $(\dot{r} \ll r)$ that come with the use of each 3-region variable $\mathfrak{r}$ by suitable $\mathcal{B} c$-formulas. Since the two conjuncts are identical in form, we only show how to deal with $(\dot{r} \ll r)$, which, we recall, is an alternative notation for $\neg C(\dot{r},-r)$. Since the complement of $-r$ is in general not connected, a direct use of notC $\left(\dot{r}+r^{\prime},(-r)+s^{\prime}\right)$ will result in an unsatisfiable formula. Instead, we represent $-r$ as the sum of two regions $s_{1}$ and $s_{2}$ with connected complements, and then proceed as before. In particular, we replace $(\dot{r} \ll r)$ by $\left((-r)=\left(s_{1}+s_{2}\right)\right) \wedge \operatorname{notC}\left(\dot{r}+r_{1}, s_{1}\right) \wedge \operatorname{notC}\left(\dot{r}+r_{2}, s_{2}\right)$. For $i=1,2$, $\dot{r}+r_{i}$ is a connected region that is disjoint from $s_{i}$. So, $\dot{r}$ is disjoint from $s_{1}$ and $s_{2}$, and hence disjoint from their sum, $-r$. Fig. 32 shows regions $r_{1}, s_{1}$ satisfying the above formula; the other pair, $r_{2}, s_{2}$ is the mirror image. 


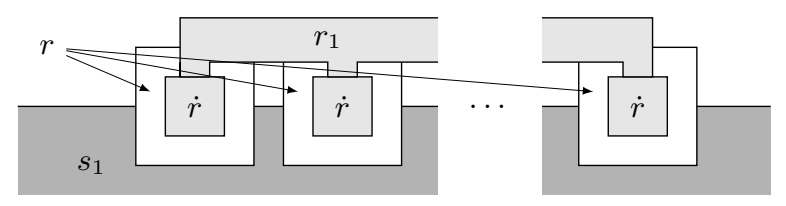

Figure 32: Disjoint connected regions $\dot{r}+r_{1}$ and $s_{1} \leq(-r)$ for $\dot{r}$ right inside $r$.

Let $\psi_{\mathrm{W}}^{c}$ be the result of replacing in $\psi_{\mathrm{W}}$ all the (explicit or implicit) conjuncts containing the predicate $C$, as just described. We have thus shown that if $\psi_{\mathrm{W}}^{c}$ is satisfiable over $R C\left(\mathbb{R}^{2}\right)$ then $W$ is positive, and, conversely, if $W$ is positive then $\psi_{\mathrm{W}}^{c}$ is satisfiable over $\operatorname{RCP}\left(\mathbb{R}^{2}\right)$. This completes the proof.

Theorem 21. The problems $\operatorname{Sat}\left(\mathcal{B} c^{\circ}, \mathrm{RC}\left(\mathbb{R}^{2}\right)\right)$ and $\operatorname{Sat}\left(\mathcal{B} c^{\circ}, \operatorname{RCP}\left(\mathbb{R}^{2}\right)\right)$ are r.e.hard.

Proof. We begin with the $\mathcal{C} c^{\circ}$-formula $\psi_{\mathrm{W}}^{\circ}$ constructed in the proof of r.e.hardness result in Corollary 19. We proceed by eliminating occurrences of $C$. However, we cannot directly use the same Lemma 14 as in the proof of Theorem 15 because the regions in question may not necessarily be bounded. For instance, consider the formula $\left(\dot{s}_{0} \ll s_{0}\right)$, which is an alternative notation for $\neg C\left(\dot{s}_{0},-s_{0}\right)$ : although the region $\dot{s}_{0}$ in Fig. 19 is evidently bounded, $-s_{0}$ is not. We proceed as follows. Say that a region $r$ is quasi-bounded if either $r$ itself or its complement, $-r$, is bounded. Since all the polygons in the tuple satisfying $\psi_{\mathrm{W}}^{\circ}$ are quasi-bounded, we can eliminate all occurrences of $C$ from $\psi_{\mathrm{W}}^{\circ}$ using the following fact [34, p. 137]:

Lemma 22. Let $F, G$ be disjoint, closed subsets of $\mathbb{R}^{2}$ such that both $\mathbb{R}^{2} \backslash F$ and $\mathbb{R}^{2} \backslash G$ are connected. Then $\mathbb{R}^{2} \backslash(F \cup G)$ is connected.

So, suppose we have a conjunct $\neg C(r, s)$ in $\psi_{\mathrm{W}}^{\circ}$. We consider the following formula:

$\chi(r, s, \vec{v})=\left(r=r_{1}+r_{2}\right) \wedge\left(s=s_{1}+s_{2}\right) \wedge \bigwedge_{1 \leq i, j \leq 2}\left(\operatorname{K5m}\left(\vec{v}_{i j}\right) \wedge\left(r_{i} \leq v_{i j}^{1}\right) \wedge\left(s_{j} \leq v_{i j}^{2}\right)\right)$,

where $\vec{v}$ is a vector of variables containing $r_{1}, r_{2}, s_{1}, s_{2}$ and the $v_{i j}^{1}, \ldots, v_{i j}^{5}$, for $1 \leq i, j \leq 2$, and $\operatorname{K5m}\left(v^{1}, \ldots, v^{5}\right)$ is the formula defined before Lemma 14 By Lemma $14(i), \chi(r, s, \vec{v})$ entails $\neg C(r, s)$ over $\mathrm{RC}\left(\mathbb{R}^{2}\right)$. We also show that, conversely, if $a$ and $b$ are disjoint quasi-bounded polygons then there exists a tuple of polygons $\vec{e}$ such that $(a, b, \vec{e})$ satisfies $\chi(r, s, \vec{v})$. Indeed, it is routine to show that, for each quasi-bounded region $a$, there exist a pair of regular closed polygons $a_{1}$ and $a_{2}$ such that $a=a_{1}+a_{2}$ and both $\mathbb{R}^{2} \backslash a_{1}$ and $\mathbb{R}^{2} \backslash a_{2}$ are connected. Let $b_{1}$ and $b_{2}$ be chosen analogously for $b$. Then, for all $1 \leq i, j \leq 2$, we have $a_{i} \cap b_{j}=\emptyset$ and, by Lemma $22, \mathbb{R}^{2} \backslash\left(a_{i}+b_{j}\right)$ is connected. Thus, there exists a piecewise-linear Jordan curve in $\mathbb{R}^{2} \backslash\left(a_{i}+b_{j}\right)$ separating $a_{i}$ and $b_{j}$. By Lemma $14(i i)$, let $\vec{e}_{i j}$ be a tuple of polygons satisfying $\mathrm{K} 5 \mathrm{~m}\left(\vec{v}_{i j}\right)$ and such that 
$a_{i} \leq e_{i j}^{1}$ and $b_{j} \leq e_{i j}^{2}$. It should be clear that the tuple of $a_{1}, a_{2}, b_{1}, b_{2}$ and the $\vec{e}_{i j}$, for $1 \leq i, j \leq 2$, is as required.

By replacing all occurrences of $C$ in $\psi_{\mathrm{w}}^{\circ}$ as described above, we obtain a $\mathcal{B} c^{\circ}$-formula, say $\psi_{\mathrm{W}}^{*}$, such that, if $\psi_{\mathrm{W}}^{*}$ is satisfiable over $\mathrm{RC}\left(\mathbb{R}^{2}\right)$, then $\mathrm{W}$ is a positive instance of PCP; and, conversely, if $\mathrm{W}$ is a positive instance of PCP, then $\psi_{\mathrm{W}}^{*}$ is satisfiable over $\mathrm{RCP}\left(\mathbb{R}^{2}\right)$.

\section{The language $\mathcal{B} c^{\circ}$ in dimensions greater than 2}

In this section, we consider the complexity of satisfying $\mathcal{B} c^{\circ}$-formulas by regular closed polyhedra and regular closed sets in three-dimensional Euclidean space. We proceed by analysing the connections between geometrical and graphtheoretic interpretations of $\mathcal{B} c^{\circ}$.

A topological space $T$ in which the intersection of any family of open sets is open is called an Aleksandrov space. Every quasi-order $(W, R)$, that is, a transitive and reflexive relation $R$ on $W$, can be regarded as an Aleksandrov space by taking $X \subseteq W$ to be open just in case $x \in X$ and $x R y$ imply $y \in X$. (Hence, $X$ is closed just in case $x \in X$ and $y R x$ implies $y \in X$.) It can be shown [6] that every Aleksandrov space is the homeomorphic image of one constructed in this way. In the sequel, we shall silently treat any quasi-order $(W, R)$ as a topological space.

It turns out that, to satisfy all satisfiable $\mathcal{B} c$ - and $\mathcal{B} c^{\circ}$-formulas, Aleksandrov spaces of rather primitive structure are enough. Call a quasi-order $\left(W_{0} \cup W_{1}, R\right)$ a quasi-saw if $W_{0}$ and $W_{1}$ are disjoint and $R$ is the reflexive closure of a relation $R^{\prime} \subseteq W_{1} \times W_{0}$ with domain $W_{1}$. The points in $W_{i}$ are said to be of depth $i$ in $\left(W_{0} \cup W_{1}, R\right)$; see Fig. 33 .

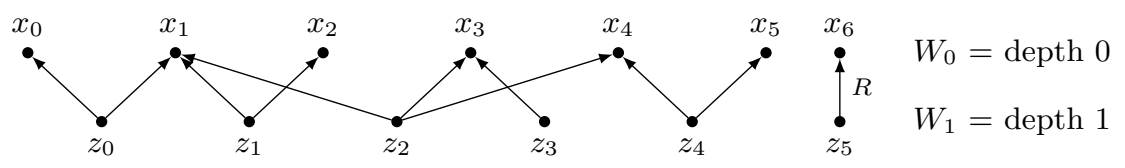

Figure 33: Quasi-saw.

Every regular closed set $X$ in a quasi-saw $\left(W_{0} \cup W_{1}, R\right)$ is uniquely defined by its points of depth 0 :

$$
\text { for each } z \in W_{1}, \quad z \in X \quad \text { iff } \quad \text { there is } x \in W_{0} \cap X \text { such that } z R x \text {. }
$$

A quasi-saw model is a model based on a quasi-saw (with variables interpreted by regular closed sets). The proof of the following lemma follows from [27, Lemmas 4.1 and 4.2] (see also [53]). But the critical observation can, in essence, be found already in [33] and [30]: for every formula $\varphi$ and every topological 
model $\mathfrak{I}$, there exist a finite Aleksandrov model $\mathfrak{A}$ and a continuous function $f: \mathfrak{I} \rightarrow \mathfrak{A}$ such that $\tau^{\mathfrak{A}}=f\left(\tau^{\mathfrak{I}}\right)$ for every term $\tau$ in $\varphi$.

Lemma 23. Let $\varphi$ be a $\mathcal{B} c$ - or $\mathcal{B} c^{\circ}$-formula. If $\varphi$ is satisfiable over $\mathrm{RC}$ then it can be satisfied in a finite quasi-saw model.

We begin by briefly discussing the results of 28 for the polyhedral case. Denote by ConRC the class of all frames over connected topological spaces with regular closed regions. For a $\mathcal{B} c^{\circ}$-formula $\varphi$, let $\varphi^{\bullet}$ be the result of replacing every occurrence of $c^{\circ}$ in $\varphi$ with $c$. Evidently, the mapping $\varphi \mapsto \varphi^{\bullet}$ is a bijection from $\mathcal{B} c^{\circ}$ to $\mathcal{B} c$.

Theorem 24. For all $n \geq 3$, the mapping $\varphi \mapsto \varphi^{\bullet}$ constitutes a reduction of $\operatorname{Sat}\left(\mathcal{B} c^{\circ}, \operatorname{RCP}\left(\mathbb{R}^{n}\right)\right)$ to $\operatorname{Sat}(\mathcal{B} c, \operatorname{ConRC})$. Hence, the problems $\operatorname{Sat}\left(\mathcal{B} c^{\circ}, \operatorname{RCP}\left(\mathbb{R}^{n}\right)\right)$ coincide, and are all EXPTIME-complete.

Proof. A connected partition in $\operatorname{RCP}\left(\mathbb{R}^{n}\right)$ is a tuple $X_{1}, \ldots, X_{k}$ of non-empty polyhedra having connected and pairwise disjoint interiors, which sum to the entire space $\mathbb{R}^{n}$. The neighbourhood graph $(V, E)$ of this partition has vertices $V=\left\{X_{1}, \ldots, X_{k}\right\}$ and edges

$$
E=\left\{\left(X_{i}, X_{j}\right) \mid i \neq j \text { and }\left(X_{i}+X_{j}\right)^{\circ} \text { is connected }\right\} ;
$$

see Fig. 34. Clearly, every connected partition in $\operatorname{RCP}\left(\mathbb{R}^{n}\right)$ has a connected
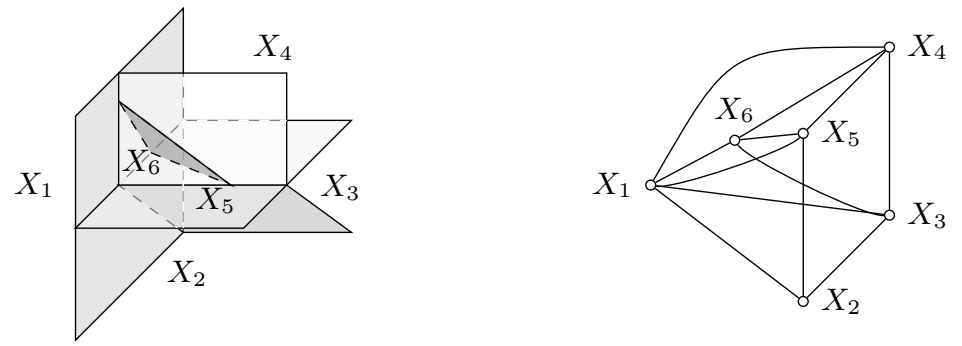

Figure 34: A connected partition in $\operatorname{RCP}\left(\mathbb{R}^{3}\right)$ and its neighbourhood graph.

neighbourhood graph; and conversely, one can show that every connected graph is the neighbourhood graph of some connected partition in $\operatorname{RCP}\left(\mathbb{R}^{n}\right)$. Furthermore, every neighbourhood graph $(V, E)$ gives rise to a quasi-saw $\left(W_{0} \cup W_{1}, R\right)$, where $W_{0}=V, W_{1}=\left\{z_{x, y} \mid(x, y) \in E\right\}$, and $R$ is the reflexive closure of $\left\{\left(z_{x, y}, x\right),\left(z_{x, y}, y\right) \mid(x, y) \in E\right\}$. Note that in this quasi-saw every point of depth 1 has precisely two $R$-successors. Such quasi-saws are called 2-quasisaws. Conversely, every connected 2-quasi-saw $\left(W_{0} \cup W_{1}, R\right)$ can be represented as the neighbourhood graph $\left(W_{0}, E\right)$ of some connected partition, where

$$
E=\left\{(x, y) \mid x \neq y \text { and there is } z \in W_{1} \text { with } z R x \text { and } z R y\right\} .
$$


From this, we see that a $\mathcal{B} c^{\circ}$-formula $\varphi$ is satisfiable over $\operatorname{RCP}\left(\mathbb{R}^{n}\right)$ if and only if $\varphi$ is satisfiable over a connected 2-quasi-saw. But, over 2-quasi-saws, connectedness coincides with interior-connectedness. Thus, $\varphi$ is satisfiable over $\operatorname{RCP}\left(\mathbb{R}^{n}\right)$ if and only if $\varphi^{\bullet}$ is satisfiable over a connected 2-quasi-saw. The problem $\operatorname{Sat}(\mathcal{B} c$, ConRC) is known to be ExPTIME-complete [27].

Having shown that the problem $\operatorname{Sat}\left(\mathcal{B} c^{\circ}, \mathrm{RCP}\left(\mathbb{R}^{3}\right)\right)$ is ExPTIME-complete, we now turn our attention to the satisfiability of $\mathcal{B} c^{\circ}$-formulas over the complete Boolean algebra $\mathrm{RC}\left(\mathbb{R}^{3}\right)$, where the picture changes drastically: for instance, the $\mathcal{B} c^{\circ}$-formula $(3)$ is not satisfiable over 2-quasi-saws, but has a quasi-saw model as in Fig. 35. In fact, it is shown in [28] that every $\mathcal{B} c^{\circ}$-formula $\varphi$ satisfiable

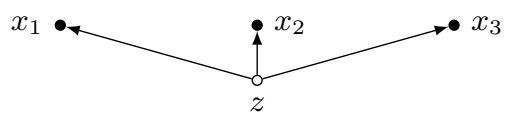

Figure 35: A quasi-saw model $\mathfrak{A}$ of $(3): r_{i}^{\mathfrak{A}}=\left\{x_{i}, z\right\}$.

over ConRC can be satisfied in a connected quasi-saw model of size bounded by a polynomial function of $|\varphi|$, and thus the problem $\operatorname{Sat}\left(\mathcal{B} c^{\circ}\right.$, ConRC) is NPcomplete. The following theorem says, in essence, that such polynomial models also give rise to 'small' models over regular closed subsets of $\mathbb{R}^{n}$, for $n \geq 3$ :

Theorem 25. The problems $\operatorname{Sat}\left(\mathcal{B} c^{\circ}, \mathrm{RC}\left(\mathbb{R}^{n}\right)\right)$, for all $n \geq 3$, coincide with $\operatorname{Sat}\left(\mathcal{B} c^{\circ}\right.$, ConRC) and are all NP-complete.

Proof. We need only establish the special case $n=3$; the general result follows by cylindrification. So, suppose $\varphi$ is satisfied in a model $\mathfrak{A}$ over a connected quasi-saw $\left(W_{0} \cup W_{1}, R\right)$ of size bounded by a polynomial function of $|\varphi|$. Let $W_{i}$ be the set of points of depth $i=0,1$. Without loss of generality we may assume that there is a point $z_{0} \in W_{1}$ with $z_{0} R x$ for all $x \in W_{0}$ (adding such a point cannot change the truth-values of subformulas of $\varphi$ of the form $\left(\tau_{1}=\tau_{2}\right)$ or $\left.c^{\circ}(\tau)\right)$. We show now how $\mathfrak{A}$ can be embedded into a model over $\operatorname{RC}\left(\mathbb{R}^{3}\right)$. In the remainder of this proof, we repeatedly rely on the fact that, if $r$ and $s$ are interior-connected, regular closed subsets of some topological space, with $r \cdot s \neq 0$, then $r+s$ is also interior-connected.

We select open balls $D_{z}$ for $z \in W_{1} \backslash\left\{z_{0}\right\}$ such that their closures are pairwise non-intersecting, and define $D_{z_{0}}=\mathbb{R}^{3} \backslash \bigcup_{z \in W_{1} \backslash\left\{z_{0}\right\}} D_{z}^{-}$. Thus, each $D_{z}$ is connected and open, and the open set $D=\bigcup_{z \in W_{1}} D_{z}$ is dense. Then we take pairwise disjoint sets $B_{x}^{1}$ for $x \in W_{0}$, each homeomorphic to a closed ball, and arranged so that, for all $x \in W_{0}$ and $z \in W_{1}, D_{z} \nsubseteq B_{x}^{1}$ and $B_{x}^{1} \cap D_{z} \neq \emptyset$ if and only if $z R x$.

We describe a construction in which the regular closed sets $B_{x}^{1}$ are expanded to sets $B_{x}$ so as to exhaust the entire space, $\mathbb{R}^{3}$. First, let $q_{1}, q_{2}, \ldots$ be an enumeration of all the points in $D$ with rational coordinates. Consider any piecewise-linear Jordan arc $\alpha$ such that the endpoints of each linear segment of $\alpha$ have rational coordinates: call such an $\alpha$ rational piecewise-linear; and let 
$\alpha_{1}, \alpha_{2}, \ldots$ be an enumeration of all the rational piecewise-linear arcs with both endpoints in the open set $D$. We define, for all $k \geq 1$, a collection $\left\{B_{x}^{k} \mid x \in W_{0}\right\}$ of interior-connected, pairwise disjoint, regular closed sets in $\mathbb{R}^{3}$. The case $k=1$ has already been dealt with. Suppose, then, for $k \geq 1$, the sets $B_{x}^{k}$ have been defined; we construct the sets $B_{x}^{k+1}$ in two steps:

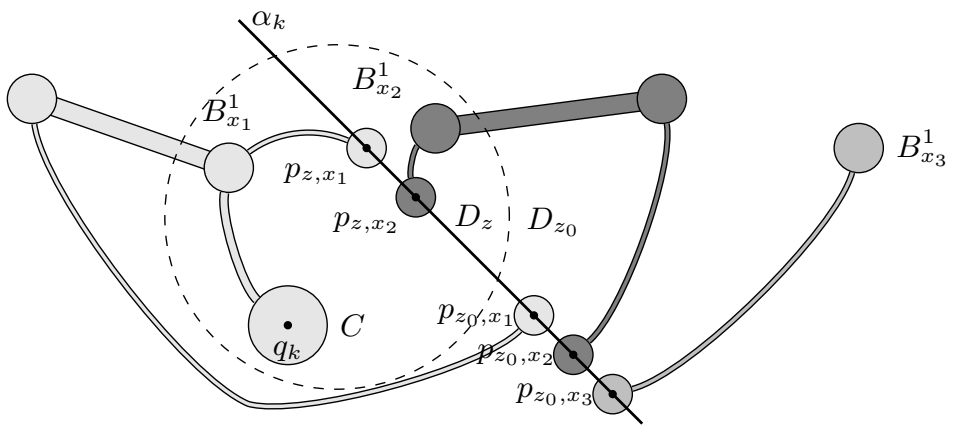

Figure 36: Filling $D_{z}$ and $D_{z_{0}}$ with the sets $B_{x_{i}}$, for $z R x_{1}, z R x_{2}$ and $z_{0} R x_{i}$, $i=1,2,3$.

1. If $q_{k} \in B_{x}^{k}$ for some $x \in W_{0}$, let $\hat{B}_{x^{\prime}}^{k}=B_{x^{\prime}}^{k}$, for every $x^{\prime} \in W_{0}$. Otherwise, $q_{k} \in D_{z}$ for some $z \in W_{1}$. Pick some $x \in W_{0}$ with $z R x$ and let $C \subseteq D_{z}$ be a regular closed interior-connected set containing $q_{k}$ and a point in $\left(B_{x}^{1}\right)^{\circ} \cap D_{z}$ in its interior (e.g., a closed ball centred on $q_{k}$ and a regular closed 'rod' connecting it to $B_{x}^{1}$, as depicted in Fig. 36. Let $\hat{B}_{x}^{k}=B_{x}^{k}+C$, and let $\hat{B}_{x^{\prime}}^{k}=B_{x^{\prime}}^{k}$ for all other $x^{\prime} \in W_{0}$. The sets $B_{x^{\prime}}^{k}$, for $x^{\prime} \in W_{0}$, are interior-connected, and $C$ can be chosen so that the $\hat{B}_{x^{\prime}}^{k}$ are pairwise disjoint.

2. Let $\hat{B}^{k}=\bigcup_{x \in W_{0}} \hat{B}_{x}^{k}$. For each $z \in W_{1}$ such that $\alpha_{k} \cap D_{z}$ is not contained in $\hat{B}^{k}$ and for each $x \in W_{0}$ such that $z R x$, choose a distinct point $p_{z, x} \in$ $\alpha_{k} \cap D_{z}$, not lying in $\hat{B}^{k}$. If $p_{z, x}$ is defined, let $C_{z, x} \subseteq D_{z}$ be a regular closed interior-connected set containing $p_{z, x}$ and a point in $\left(B_{x}^{k}\right)^{\circ} \cap D_{z}$ in its interior, see Fig. 36. otherwise, let $C_{z, x}=\emptyset$. Set $B_{x}^{k+1}=\hat{B}_{x}^{k}+\sum_{z \in W_{1}} C_{z, x}$, for all $x \in W_{0}$. The sets $B_{x}^{k+1}$ are interior-connected and, clearly, the $C_{z, x}$ can be chosen such that the $B_{x}^{k+1}$ are also pairwise disjoint.

This completes the definition of the sets $\left\{B_{x}^{k} \mid x \in W_{0}\right\}$ for all $k \geq 1$.

Since $\mathrm{RC}\left(\mathbb{R}^{3}\right)$ is a complete Boolean algebra, define $B_{x}=\sum_{k=1}^{\infty} B_{x}^{k}$, for each $x \in W_{0}$. We show that the sets $B_{x}$ are interior-connected and form a partition (i.e., their pairwise products are empty, and they sum to $\mathbb{R}^{3}$ ). Indeed, for distinct $x, y \in W_{0}$, we certainly have, for all $k, \ell \geq 1, B_{x}^{k} \cdot B_{y}^{\ell}=0$, whence, by the distributivity law, $B_{x} \cdot B_{y}=0$. And since, for all $k \geq 1, B_{x}^{k}$ is interior-connected and includes the non-empty, interior-connected set $B_{x}^{1}$, the set $\bigcup_{k=1}^{\infty}\left(B_{x}^{k}\right)^{\circ}$ is 
connected. But $B_{x}^{\circ}$ lies in between $\bigcup_{k=1}^{\infty}\left(B_{x}^{k}\right)^{\circ}$ and its closure, and hence is also connected. Finally, by Step 1 of the above construction, every rational point of the set $D$ lies in some $B_{x}$, so that $\sum_{x \in W_{0}} B_{x} \supseteq D$, whence $\sum_{x \in W_{0}} B_{x}=1$. This completes the definition of the partition $\left\{B_{x} \mid x \in W_{0}\right\}$.

Now define a function $f: \operatorname{RC}(W, R) \rightarrow \mathrm{RC}\left(\mathbb{R}^{3}\right)$ by

$$
f(X)=\sum_{x \in X \cap W_{0}} B_{x}
$$

Let $X \in \mathrm{RC}(W, R)$, and let $z$ be a point of $W_{1}$. We claim that $z \in X^{\circ}$ implies $D_{z} \subseteq f(X)$; further, if $z R x$, then $D_{z}+B_{x}$ is interior-connected. Indeed, if $z \in X^{\circ}$, with $z R x$, then $x \in X$. And since, by Step 1 of the above construction, every rational point of $D_{z}$ lies in $B_{x}$ for some such $x$, it follows that $D_{z} \subseteq$ $\sum\left\{B_{x} \mid x \in X \cap W_{0}\right\}=f(X)$. The second statement follows easily from the choice of the sets $B_{x}^{1}$ and the fact that the sets $B_{x}$ are interior-connected.

We are now ready to show that $f$ is a Boolean algebra homomorphism, and that $X \in \mathrm{RC}(W, R)$ is interior-connected if and only if $f(X)$ is interiorconnected. Trivially, $f(X+Y)=f(X)+f(Y)$; and since the $B_{x}$ form a partition, $f(-X)=\sum_{x \in W_{0} \backslash X} B_{x}=-f(X)$. Now suppose $X \in \mathrm{RC}(W, R)$ is interior-connected, and let $p, q$ be points in $f(X)^{\circ}$. Then there exist points $p^{\prime}, q^{\prime}$ in the same components of $f(X)^{\circ}$ as $p, q$, respectively, such that, for some $k \geq 1$ and $x, y \in X \cap W_{0}$, we have $p^{\prime} \in B_{x}^{k}$ and $q^{\prime} \in B_{y}^{k}$. Since $X$ is interior-connected, we can find sequences of points $x_{0}, \ldots, x_{m}$ in $X \cap W_{0}$, and $z_{1}, \ldots, z_{m} \in X^{\circ} \cap W_{1}$ such that $x=x_{0}, y=x_{m}$ and $z_{i} R x_{i-1}$ and $z_{i} R x_{i}$, for all $1 \leq i \leq m$. But we have shown above that the sets $D_{z_{i}}$ are subsets of $f(X)$, and that the sets $D_{z_{i}}+B_{x_{i}}$ and $D_{z_{i}}+B_{x_{i-1}}$ are interior-connected. Hence, $p^{\prime}$ and $q^{\prime}$ lie in the same component of $f(X)^{\circ}$, whence $p$ and $q$ do as well. That is: $f(X)$ is interior-connected, as required. Finally, suppose $X \in \mathrm{RC}(W, R)$ is not interior-connected, so that we may find elements $x, y \in W_{0}$ lying in different components of $X^{\circ}$. We show that $f(X)$ is not interior-connected. For suppose otherwise. Then there exists a rational piecewise-linear arc $\alpha$ with endpoints in the sets $D \cap\left(B_{x}^{1}\right)^{\circ}$ and $D \cap\left(B_{y}^{1}\right)^{\circ}$, and lying entirely in $f(X)^{\circ}$. But $\alpha$ occurs as some $\alpha_{k}$ in our enumeration. It follows that there will be a first point $q^{\prime}$ of $\alpha_{k}$ lying in a set $\hat{B}_{y^{\prime}}^{k}$ such that $x$ and $y^{\prime}$ lie in different components of $X^{\circ}$; and there will be a last point $p^{\prime}$ of $\alpha_{k}$, occurring strictly before $q^{\prime}$ and lying in a set $\hat{B}_{x^{\prime}}^{k}$. Obviously, $x^{\prime}$ and $y^{\prime}$ lie in different components of $X^{\circ}$. Let $\alpha^{\prime}$ be the interior segment of $\alpha_{k}$ between $p^{\prime}$ and $q^{\prime}$ (i.e. without the end-points); thus $\alpha^{\prime}$ does not intersect $\hat{B}^{k}$. By construction of the sets $D_{z}\left(z \in W_{1}\right)$, either $\alpha^{\prime}$ lies entirely in $D_{z}$ for some $z \in W_{1} \backslash\left\{z_{0}\right\}$, or $\alpha^{\prime}$ intersects $D_{z_{0}}$. In the former case, since $z R x^{\prime}$ and $z R y^{\prime}$, but $x^{\prime}$ and $y^{\prime}$ lie in different components of $X^{\circ}$, it follows that $z \notin X^{\circ}$. Thus, there exists $x^{\prime \prime} \in W_{0}$ such that $z R x^{\prime \prime}$ and $x^{\prime \prime} \notin X$. But then Step 2 in the above construction ensures that $\alpha^{\prime} \subseteq \alpha_{k}$ contains points of $B_{x^{\prime \prime}}^{k+1} \subseteq B_{x^{\prime \prime}}$, contradicting the supposition that $\alpha_{k} \subseteq f(X)^{\circ}$. On the other hand, if $\alpha^{\prime} \cap D_{z_{0}} \neq \emptyset$, then, since $X$ is not interior-connected, it follows that $X \neq 1$, and so there certainly exists $x^{\prime \prime} \in W_{0}$ such that $z_{0} R x^{\prime \prime}$ and $x^{\prime \prime} \notin X$. By the same reasoning as before, $\alpha^{\prime}$ contains points of $B_{x^{\prime \prime}}^{k+1} \subseteq B_{x^{\prime \prime}}$, contradicting 
the supposition that $\alpha_{k} \subseteq f(X)^{\circ}$.

Now simply define an interpretation $\mathfrak{I}$ over $\operatorname{RC}\left(\mathbb{R}^{3}\right)$ by setting $r^{\mathfrak{I}}=f\left(r^{\mathfrak{A}}\right)$. It immediately follows from the previous paragraph that $\varphi$ is true in $\mathfrak{I}$.

This resolves the decidability and complexity of the problems $\operatorname{Sat}\left(\mathcal{B} c^{\circ}, \operatorname{RC}\left(\mathbb{R}^{n}\right)\right)$ and $\operatorname{Sat}\left(\mathcal{B} c^{\circ}, \operatorname{RCP}\left(\mathbb{R}^{n}\right)\right)$ for all $n \geq 3$. Recall from Sec. 4 that $\operatorname{Sat}\left(\mathcal{L}, \operatorname{RCP}\left(\mathbb{R}^{n}\right)\right)$ is undecidable for all $n \geq 2$, where $\mathcal{L}$ is any of $\mathcal{B} c, \mathcal{C} c$ or $\mathcal{C} c^{\circ}$; and recall from Sec. 5 that $\operatorname{Sat}\left(\mathcal{B} c^{\circ}, \operatorname{RCP}\left(\mathbb{R}^{2}\right)\right)$ is undecidable, and that $\operatorname{Sat}\left(\mathcal{L}, \operatorname{RC}\left(\mathbb{R}^{2}\right)\right)$ is also undecidable, where $\mathcal{L}$ is any of $\mathcal{B} c, \mathcal{B} c^{\circ}, \mathcal{C} c$ or $\mathcal{C} c^{\circ}$. At the time of writing, it is not known whether any of the problems $\operatorname{Sat}\left(\mathcal{B} c, \mathrm{RC}\left(\mathbb{R}^{n}\right)\right)$, $\operatorname{Sat}\left(\mathcal{C} c, \mathrm{RC}\left(\mathbb{R}^{n}\right)\right)$ or $\operatorname{Sat}\left(\mathcal{C} c^{\circ}, \operatorname{RC}\left(\mathbb{R}^{n}\right)\right)$, for $n \geq 3$, is decidable. The best currently available lower bound can be found in [27, where all three problems are shown to be ExPTime-hard.

\section{Acknowledgements}

Roman Kontchakov and Michael Zakharyaschev acknowledge the support of the EPSRC, grant ref. EP/E034942/1. Yavor Nenov acknowledges the support of the EPSRC, DTA account FA01413. Ian Pratt-Hartmann acknowledges the support of the EPSRC, grant ref. EP/E035248/1, and expresses his thanks to the Department of Mathematics and Computer Science, University of Wrocław, and the Transregional Collaborative Research Center SFB/TR 8 "Spatial Cognition", University of Bremen, for their hospitality during the writing of this paper.

Yavor Nenov, Ian Pratt-Hartmann

School of Computer Science

University of Manchester

Manchester, M13 9PL, United Kingdom

Roman Kontchakov, Michael Zakharyaschev

Department of Computer Science and Information Systems

Birkbeck, University of London

Malet Street, London, WC1E 7HX, United Kingdom

\section{References}

[1] C. Areces, P. Blackburn, and M. Marx. The computational complexity of hybrid temporal logics. Logic Journal of the IGPL, 8:653-679, 2000.

[2] Ph. Balbiani, T. Tinchev, and D. Vakarelov. Modal logics for region-based theories of space. Fundamenta Informaticae, 81:29-82, 2007.

[3] B. Bennett. Spatial reasoning with propositional logic. In Proc. of KR, pages 51-62. Morgan Kaufmann, 1994. 
[4] L. Biacino and G. Gerla. Connection structures. Notre Dame Journal of Formal Logic, 32:242-247, 1991.

[5] J. Bochnak, M. Coste, and M.-F. Roy. Real Algebraic Geometry. Ergebnisse der Mathematik und ihrer Grenzgebiete, Vol. 36. Springer, 1998.

[6] N. Bourbaki. General Topology, Part 1. Hermann, Paris \& Addison-Wesley, 1966.

[7] B. L. Clarke. A calculus of individuals based on 'connection'. Notre Dame Journal of Formal Logic, 23:204-218, 1981.

[8] B. L. Clarke. Individuals and points. Notre Dame Journal of Formal Logic, 26:61-75, 1985.

[9] A. Cohn, B. Bennett, J. Gooday, and N. Gotts. Qualitative spatial representation and reasoning with the region connection calculus. Geoinformatica, $1(3): 275-316,1997$.

[10] A. Cohn and J. Renz. Qualitative spatial representation and reasoning. In F. van Hermelen, V. Lifschitz, and B. Porter, editors, Handbook of Knowledge Representation, pages 551-596. Elsevier, 2008.

[11] E. Davis. The expressivity of quantifying over regions. Journal of Logic and Computation, 16:891-916, 2006.

[12] T. de Laguna. Point, line and surface as sets of solids. The Journal of Philosophy, 19:449-6, 1922.

[13] G. Dimov and D. Vakarelov. Contact algebras and region-based theory of space: a proximity approach, I. Fundamenta Informaticae, 74(2-3):209$249,2006$.

[14] G. Dimov and D. Vakarelov. Contact algebras and region-based theory of space: a proximity approach, II. Fundamenta Informaticae, 74(2-3):251$282,2006$.

[15] C. Dornheim. Undecidability of plane polygonal mereotopology. In A. Cohn, L. Schubert, and S. Shapiro, editors, Proc. of KR, pages 342353. Morgan Kaufmann, 1998.

[16] I. Düntsch and M. Winter. A representation theorem for Boolean contact algebras. Theoretical Computer Science, 347:498-512, 2005.

[17] M. Egenhofer and R. Franzosa. Point-set topological spatial relations. International Journal of Geographical Information Systems, 5:161-174, 1991.

[18] D. Gabelaia, R. Kontchakov, A. Kurucz, F. Wolter, and M. Zakharyaschev. Combining spatial and temporal logics: expressiveness vs. complexity. Journal of Artificial Intelligence Research, 23:167-243, 2005. 
[19] K. Gödel. Eine Interpretation des intuitionistischen Aussagenkalküls. Ergebnisse eines mathematischen Kolloquiums, 4:39-40, 1933.

[20] V. Goranko and S. Passy. Using the universal modality: gains and questions. Journal of Logic and Computation, 2:5-30, 1992.

[21] A. Griffiths. Computational Properties Of Spatial Logics In The Real Plane. $\mathrm{PhD}$ thesis, School of Computer Science, University of Manchester, 2008.

[22] A. Grzegorczyk. Undecidability of some topological theories. Fundamenta Mathematicae, 38:137-152, 1951.

[23] D. Hilbert. Grundlagen der Geometrie. B.G. Teubner, Leipzig and Berlin, 1909.

[24] R. Kontchakov, Y. Nenov, I. Pratt-Hartmann, and M. Zakharyaschev. On the decidability of connectedness constraints in 2D and 3D Euclidean spaces. In T. Walsh, editor, Proc. of IJCAI, pages 957-962. AAAI Press, 2011.

[25] R. Kontchakov, I. Pratt-Hartmann, F. Wolter, and M. Zakharyaschev. On the computational complexity of spatial logics with connectedness constraints. In Proc. of LPAR, volume 5330 of LNAI, pages 574-589. Springer, 2008.

[26] R. Kontchakov, I. Pratt-Hartmann, F. Wolter, and M. Zakharyaschev. Topology, connectedness, and modal logic. In Advances in Modal Logic, vol. 7, pages 151-176. College Publications, London, 2008.

[27] R. Kontchakov, I. Pratt-Hartmann, F. Wolter, and M. Zakharyaschev. Spatial logics with connectedness predicates. Logical Methods in Computer Science, 6(3), 2010.

[28] R. Kontchakov, I. Pratt-Hartmann, and M. Zakharyaschev. Interpreting topological logics over Euclidean spaces. In Proc. of KR, pages 534-544. AAAI Press, 2010.

[29] S. Koppelberg. Handbook of Boolean Algebras, volume 1. North-Holland, 1989.

[30] S. Kripke. Semantical analysis of modal logic I: Normal modal propositional calculi. Zeitschrift für Mathematische Logik und Grundlagen der Mathematik, 9:67-96, 1963.

[31] C. Kuratowski. Sur la structure des frontières communes à deux régions. Fundamenta Mathematicae, 12:20-42, 1928.

[32] C. Lutz and F. Wolter. Modal logics of topological relations. Logical Methods in Computer Science, 2, 2006. 
[33] J.C.C. McKinsey and A. Tarski. The algebra of topology. Annals of Mathematics, 45:141-191, 1944.

[34] M.H.A. Newman. Elements of the Topology of Plane Sets of Points. Cambridge, 1964.

[35] W. Nutt. On the translation of qualitative spatial reasoning problems into modal logics. In W. Burgard, T. Christaller, and A. Cremers, editors, Proc. of KI, volume 1701 of $L N C S$, pages 113-124. Springer, 1999.

[36] I. Orlov. The calculus of compatibility of propositions. Mathematics of the USSR, Sbornik, 35:263-286, 1928. (In Russian).

[37] E. Post. A variant of a recursively unsolvable problem. Bulletin of the $A M S, 52: 264-268,1946$.

[38] I. Pratt-Hartmann. A topological constraint language with component counting. Journal of Applied Non-Classical Logics, 12:441-467, 2002.

[39] I. Pratt-Hartmann. First-order mereotopology. In M. Aiello, I. PrattHartmann, and J. van Benthem, editors, Handbook of Spatial Logics, pages 13-97. Springer, 2007.

[40] D. Randell, Z. Cui, and A. Cohn. A spatial logic based on regions and connection. In B. Nebel, C. Rich, and W. Swartout, editors, Proc. of KR, pages 165-176. Morgan Kaufmann, 1992.

[41] J. Renz. A canonical model of the region connection calculus. In A. Cohn, L. Schubert, and S. Shapiro, editors, Proc. of KR, pages 330-341. Morgan Kaufmann, 1998.

[42] J. Renz and B. Nebel. On the complexity of qualitative spatial reasoning: A maximal tractable fragment of the region connection calculus. In Proc. of IJCAI, pages 522-527, 1997.

[43] J. Renz and B. Nebel. On the complexity of qualitative spatial reasoning. Artificial Intelligence, 108:69-123, 1999.

[44] J. Renz and B. Nebel. Efficient methods for qualitative spatial reasoning. Journal of Artificial Intelligence Research, 15:289-318, 2001.

[45] M. Schaefer, E. Sedgwick, and D. Štefankovič. Recognizing string graphs in NP. Journal of Computer and System Sciences, 67:365-380, 2003.

[46] V. Shehtman. "Everywhere" and "Here". Journal of Applied Non-Classical Logics, 9:369-379, 1999.

[47] T. Smith and K. Park. An algebraic approach to spatial reasoning. International Journal of Geographical Information Systems, 6:177-192, 1992. 
[48] A. Tarski. What is Elementary Geometry? In The Axiomatic Method, with Special Reference to Geometry and Physics, pages 16-29. North-Holland, 1959.

[49] T. Tinchev and D. Vakarelov. Logics of space with connectedness predicates: Complete axiomatizations. In Advances in Modal Logic, volume 8, pages 434-453. College Publications, 2010.

[50] D. Vakarelov. Region-based theory of space: algebras of regions, representation theory, and logics. In D.M. Gabbay, S. Goncharov, and M. Zakharyaschev, editors, Mathematical Problems from Applied Logic II, pages 267-348. Springer, 2007.

[51] J. van Benthem and G. Bezhanishvili. Modal logics of space. In M. Aiello, I. Pratt-Hartmann, and J. van Benthem, editors, Handbook of Spatial Log$i c s$, pages 217-298. Springer, 2007.

[52] A. N. Whitehead. Process and Reality. New York: The MacMillan Company, 1929.

[53] F. Wolter and M. Zakharyaschev. Spatial reasoning in RCC-8 with Boolean region terms. In W. Horn, editor, Proc. of ECAI, pages 244-248. IOS Press, 2000 . 